Supporting Information

\title{
Oxyamination of Unactivated Alkenes with Electron-Rich Amines and Acids via a Catalytic Triiodide Intermediate
}

Fan $\mathrm{Wu}^{\dagger}$, Jeewani Poornima Ariyarathna ${ }^{\dagger}$, Nur-E Alom, Navdeep Kaur, and Wei Li*

${ }^{\dagger}$ These authors contributed equally to this work.

Department of Chemistry and Biochemistry, School of Green Chemistry and Engineering, The University of Toledo, 2801 West Bancroft Street, Toledo, Ohio 43606, United States

E-mail: Wei.Li@UToledo.edu 


\section{Content}

1. General Information............................................ 3

2. Experimental Procedures...................................... 44

3. Spectral Characterization of the Products................... S4-S30

4. References ............................................... S31

5. Spectral Data ............................................ S32-S71 


\section{General Information.}

Commercial reagents and solvents were purchased from Sigma Aldrich, Oakwood Chemicals, Alfa Aesar, Matrix Scientific, Acros Organic, and were used as received. Alkenoic acid substrates for products $3,{ }^{1} 19,{ }^{2} 20,{ }^{2} 21,{ }^{2} 22,{ }^{2} 23,{ }^{2} 24,{ }^{2} 26,{ }^{2} 28,{ }^{3} 29,{ }^{3} 31,{ }^{1} 32,{ }^{1} 33,{ }^{4} 34,{ }^{1} 27^{5}$ were synthesized according to reported procedures. Organic solutions were concentrated under reduced pressure on a IKA rotary evaporator using an acetone-dry ice bath. Chromatographic purification of products was accomplished using flash chromatography on 230-400 mesh silica gel. Thin-layer chromatography (TLC) was performed on Analtech $250 \mathrm{~mm}$ silica gel HLF UV-254 plates. Visualization of the developed plates was performed by fluorescence quenching, potassium permanganate and iodine-silica gel system. ${ }^{1} \mathrm{H}$ and ${ }^{13} \mathrm{C}$ NMR spectra were recorded on a Bruker 600 instrument $(600$ and $150 \mathrm{MHz})$ or INOVA $600(600$ and $150 \mathrm{MHz})$ and are internally referenced to residual protio solvent signals (for $\mathrm{CDCl}_{3}, 7.26$ and $77.0 \mathrm{ppm}$, respectively). Data for ${ }^{1} \mathrm{H}$ NMR were reported as follows: chemical shift $(\mathrm{ppm})$, multiplicity $(\mathrm{s}=$ singlet, $\mathrm{d}=$ doublet, $\mathrm{t}=$ triplet, $\mathrm{q}=$ quartet, $\mathrm{h}=$ heptet, $\mathrm{m}=$ multiplet, $\mathrm{br}=$ broad$)$, integration, coupling constant $(\mathrm{Hz})$. ${ }^{13} \mathrm{C}$ spectra were reported as chemical shifts in ppm and multiplicity where appropriate. IR spectra were recorded on a PerkinElmer FT-IR spectrophotometer and reported in terms of wavenumber

of absorption $\left(\mathrm{cm}^{-1}\right)$. High resolution mass spectra were obtained on Waters Synapt High Definition Mass Spectrometer (HDMS) by electrospray ionization at University of Toledo, $\mathrm{OH}$, USA. 


\section{Experimental Procedures}

\section{General Procedure for Lactam Synthesis:}

To a $8 \mathrm{~mL}$ vial equipped with a stir bar was added $\mathrm{Cu}(\mathrm{OTf})_{2}(18 \mathrm{mg}, 0.05 \mathrm{mmol})$ and $\mathrm{KI}(25 \mathrm{mg}$, $0.15 \mathrm{mmol})$, the vial was then evacuated and backfilled with oxygen 3 times. Then solvent $(\mathrm{MeOH}$, $2.5 \mathrm{~mL})$ was added via syringe, followed by alkenoic acids $(0.5 \mathrm{mmol})$ and amines $(1.5 \mathrm{mmol})$. The reaction mixture was then heated to $60^{\circ} \mathrm{C}$ with heating block and stirred for $24 \mathrm{~h}$. After cooling to room temperature, the reaction mixture was diluted with EtOAc $(5 \mathrm{~mL})$ and saturated aqueous $\mathrm{NH}_{4} \mathrm{Cl}$ solution $(1 \mathrm{~mL})$ was then added. The organic layer was separated and the aqueous layer was extracted with EtOAc $(2 \times 2 \mathrm{~mL})$. The combined organic layer was dried over $\mathrm{Na}_{2} \mathrm{SO}_{4}$ and concentrated under reduced pressure to give the crude product, which was purified by column chromatography on silica gel to afford the pure product.

\section{Spectral Characterization of the Products}

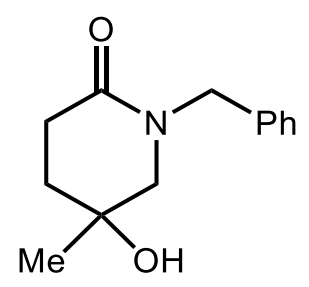

1-benzyl-5-hydroxy-5-methylpiperidin-2-one (3): This compound was prepared according to the General Procedure using 4-methylpent-4-enoic acid ( $57 \mathrm{mg}, 0.5 \mathrm{mmol})$, benzylamine (160 $\mathrm{mg}$ $1.5 \mathrm{mmol})$. After purification by column chromatography $\mathrm{SiO}_{2}(50 \%$ EtOAc/Hexane to $5 \%$ $\mathrm{MeOH} / \mathrm{EtOAc}$ ), the title compound was isolated as a white solid (81 mg, 74\% yield).

${ }^{1} \mathrm{H}$ NMR (600 MHz, DCCl $)$ ) 7.32-7.26 (m, $\left.2 \mathrm{H}\right), 7.26-7.20$ (m, $\left.3 \mathrm{H}\right), 4.58-4.51$ (m, $\left.2 \mathrm{H}\right), 3.15$ (d, $J=12.7 \mathrm{~Hz}, 1 \mathrm{H}), 3.05(\mathrm{dd}, J=12.7,2.0 \mathrm{~Hz}, 1 \mathrm{H}), 2.73-2.63(\mathrm{~m}, 1 \mathrm{H}), 2.46-2.38(\mathrm{~m}, 1 \mathrm{H}), 2.02$ 
(br s, $1 \mathrm{H}), 1.88-1.74(\mathrm{~m}, 2 \mathrm{H}), 1.24(\mathrm{~s}, 3 \mathrm{H}) ;{ }^{13} \mathrm{C} \mathrm{NMR}\left(150 \mathrm{MHz}, \mathrm{CDCl}_{3}\right) \delta$ 169.4, 136.8, 128.6, 128.0, 127.4, 67.2, 58.4, 50.1, 33.8, 28.5, 27.3. IR (neat): 3293, 3062, 3021, 2930, 1612, 1503 , 1314, 1220, 1125, 1031, $734 \mathrm{~cm}-1$; HRMS (ESI) m/z calcd for $\mathrm{C}_{13} \mathrm{H}_{18} \mathrm{NO}_{2}\left[(\mathrm{M}+\mathrm{H})^{+}\right] 220.1338$, found 220.1328 .<smiles>COc1cccc(CN2CC(C)(O)CCC2=O)c1</smiles>

5-hydroxy-1-(3-methoxy)-5-methylpiperidin-2-one (4): This compound was prepared according to the General Procedure using 4-methylpent-4-enoic acid (57 mg, $0.5 \mathrm{mmol}$ ), (3methoxyphenyl)methanamine (206 $\mathrm{mg}, 1.5 \mathrm{mmol}$ ). After purification by column chromatography $\mathrm{SiO}_{2}(50 \% \mathrm{EtOAc} / \mathrm{Hexane}$ to $10 \% \mathrm{MeOH} / \mathrm{EtOAc})$, the title compound was isolated as an orange solid (97 mg, 78\% yield).

${ }^{1} \mathrm{H}$ NMR $\left(600 \mathrm{MHz}, \mathrm{CDCl}_{3}\right): \delta 7.20(\mathrm{t}, J=7.5 \mathrm{~Hz}, 1 \mathrm{H}), 6.82-6.74(\mathrm{~m}, 3 \mathrm{H}), 4.59(\mathrm{~d}, J=14.7 \mathrm{~Hz}$, $1 \mathrm{H}), 4.42(\mathrm{~d}, J=14.9 \mathrm{~Hz}, 1 \mathrm{H}), 3.75(\mathrm{~s}, 3 \mathrm{H}), 3.25(\mathrm{br} \mathrm{s}, 1 \mathrm{H}), 3.14(\mathrm{~d}, J=12.9 \mathrm{~Hz}, 1 \mathrm{H}), 3.08(\mathrm{~d}$, $J=12.9 \mathrm{~Hz}, 1 \mathrm{H}), 2.71-2.63(\mathrm{~m}, 1 \mathrm{H}), 2.42-2.35(\mathrm{~m}, 1 \mathrm{H}), 1.88-1.81(\mathrm{~m}, 1 \mathrm{H}), 1.80-1.72(\mathrm{~m}, 1 \mathrm{H})$, $1.24(\mathrm{~s}, 3 \mathrm{H}) ;{ }^{13} \mathrm{C} \mathrm{NMR}\left(150 \mathrm{MHz}, \mathrm{CDCl}_{3}\right): \delta$ 169.6, 159.7, 138.3, 129.5, 120.1, 113.3, 112.7, 66.9, 58.4, 55.1, 50.0, 33.7, 28.5, 27.3; IR (neat): 3248, 2969, 2932, 1600, 1492, 1253, 1162, 1036, 692 $\mathrm{cm}^{-1}$; HRMS (ESI) m/z calcd for $\mathrm{C}_{14} \mathrm{H}_{20} \mathrm{NO}_{3}\left[(\mathrm{M}+\mathrm{H})^{+}\right] 250.1433$, found 250.1455 .<smiles>CC1(O)CCC(=O)N(Cc2ccc(Br)cc2)C1</smiles> 
1-(4-bromobenzyl)-5-hydroxy-5-methylpiperidin-2-one (5): This compound was prepared according to the General Procedure using 4-methylpent-4-enoic acid (57 mg, $0.5 \mathrm{mmol})$, (4bromophenyl)methanamine (279 mg $1.5 \mathrm{mmol})$. After purification by column chromatography $\mathrm{SiO}_{2}(50 \% \mathrm{EtOAc} / \mathrm{Hexane}$ to $5 \% \mathrm{MeOH} / \mathrm{EtOAc})$, the title compound was isolated as a white solid (125 mg, 84\% yield).

${ }^{1} \mathrm{H}$ NMR $\left(600 \mathrm{MHz}, \mathrm{CDCl}_{3}\right) \delta 7.41(\mathrm{~d}, J=8.3 \mathrm{~Hz}, 2 \mathrm{H}), 7.11(\mathrm{~d}, J=8.3 \mathrm{~Hz}, 2 \mathrm{H}), 4.53-4.42(\mathrm{~m}$, $2 \mathrm{H}), 3.14(\mathrm{~d}, J=12.0 \mathrm{~Hz}, 1 \mathrm{H}), 3.06(\mathrm{dd}, J=12.7,2.0 \mathrm{~Hz}, 1 \mathrm{H}), 2.72-2.63(\mathrm{~m}, 2 \mathrm{H}), 2.43-2.35$ (m, $1 \mathrm{H}), 1.89-1.82(\mathrm{~m}, 1 \mathrm{H}), 1.82-1.74(\mathrm{~m}, 1 \mathrm{H}), 1.25(\mathrm{~s}, 3 \mathrm{H}) ;{ }^{13} \mathrm{C} \mathrm{NMR}\left(150 \mathrm{MHz}, \mathrm{CDCl}_{3}\right) \delta 169.7$, 135.8, 131.6, 129.6, 121.2, 66.9, 58.4, 49.5, 33.6, 28.4, 27.3; IR (neat): 3515, 3463, 1595, 1508, 1489, 1463, 1280, $1085 \mathrm{~cm}^{-1}$; HRMS (ESI) $\mathrm{m} / z$ calcd for $\mathrm{C}_{13} \mathrm{H}_{17} \mathrm{BrNO}_{2}\left[(\mathrm{M}+\mathrm{H})^{+}\right] 298.0443$, found 298.0446.

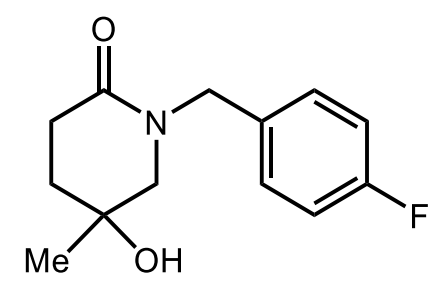

1-(4-fluorobenzyl)-5-hydroxy-5-methylpiperidin-2-one (6): This compound was prepared according to the General Procedure using 4-methylpent-4-enoic acid (57 mg, $0.5 \mathrm{mmol}$ ), (4fluorophenyl)methanamine (188 $\mathrm{mg}, 1.5 \mathrm{mmol})$. After purification by column chromatography $\mathrm{SiO}_{2}(100 \%$ EtOAc to $5 \% \mathrm{MeOH} / \mathrm{EtOAc})$, the title compound was isolated as a colorless oil (85 mg, $71 \%$ yield).

${ }^{1} \mathrm{H}$ NMR (600 MHz, $\left.\mathrm{CDCl}_{3}\right): \delta$ 7.24-7.16 (m, $\left.2 \mathrm{H}\right), 7.00-6.94(\mathrm{~m}, 2 \mathrm{H}), 4.53(\mathrm{~d}, J=14.6 \mathrm{~Hz}, 1 \mathrm{H})$, $4.415(\mathrm{~d}, J=14.6 \mathrm{~Hz}, 1 \mathrm{H}), 3.19(\mathrm{br} \mathrm{s}, 1 \mathrm{H}), 3.13(\mathrm{~d}, J=12.5 \mathrm{~Hz}, 1 \mathrm{H}), 3.07$ (d, $J=12.5 \mathrm{~Hz}, 1 \mathrm{H})$, 2.71-2.63 (m, $1 \mathrm{H}), 2.43-2.33(\mathrm{~m}, 1 \mathrm{H}), 1.91-1.72(\mathrm{~m}, 2 \mathrm{H}), 1.24(\mathrm{~s}, 3 \mathrm{H}) ;{ }^{13} \mathrm{C} \mathrm{NMR}(150 \mathrm{MHz}$, 
$\left.\mathrm{CDCl}_{3}\right): \delta 169.7,162.0\left(\mathrm{~d}, J_{\mathrm{C}, \mathrm{F}}=244.5 \mathrm{~Hz}\right), 132.4\left(\mathrm{~d}, J_{\mathrm{C}, \mathrm{F}}=3.5 \mathrm{~Hz}\right), 129.6\left(\mathrm{~d}, J_{\mathrm{C}, \mathrm{F}}=8.1 \mathrm{~Hz}\right), 115.3$ $\left(\mathrm{d}, J_{\mathrm{C}, \mathrm{F}}=21.2 \mathrm{~Hz}\right), 66.8,58.3,49.4,33.6,28.4,27.2$; IR (neat): 3299, 2961, 2922, 1605, 1503, 1242, 1156, 848, $514 \mathrm{~cm}^{-1}$; HRMS (ESI) $\mathrm{m} / z$ calcd for $\mathrm{C}_{13} \mathrm{H}_{17} \mathrm{FNO}_{2}\left[(\mathrm{M}+\mathrm{H})^{+}\right] 238.1243$, found 238.1249 .

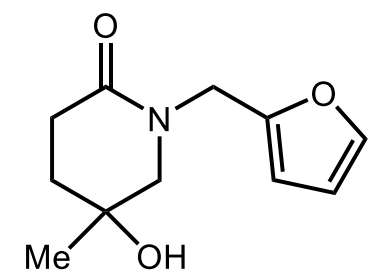

1-(furan-2-ylmethyl)-5-hydroxy-5-methylpiperidin-2-one (7): This compound was prepared according to the General Procedure using 4-methylpent-4-enoic acid (57 mg, $0.5 \mathrm{mmol}$ ), furfurylamine (146 mg, $1.5 \mathrm{mmol})$. After purification by column chromatography $\mathrm{SiO}_{2}(100 \%$ EtOAc to $10 \% \mathrm{MeOH} / \mathrm{EtOAc}$ ), the title compound was isolated as a colorless oil (28 mg, $27 \%$ yield).

${ }^{1} \mathrm{H}$ NMR (600 MHz, CDCl $): \delta 7.36$ (s, $\left.1 \mathrm{H}\right), 6.36-6.25(\mathrm{~m}, 2 \mathrm{H}), 4.68(\mathrm{~d}, J=15.4 \mathrm{~Hz}, 1 \mathrm{H}), 4.45$ $(\mathrm{d}, J=15.4 \mathrm{~Hz}, 1 \mathrm{H}), 3.28(\mathrm{~d}, J=12.6 \mathrm{~Hz}, 1 \mathrm{H}), 3.21(\mathrm{~d}, J=12.6 \mathrm{~Hz}, 1 \mathrm{H}), 2.71-2.61(\mathrm{~m}, 1 \mathrm{H})$, 2.49-2.38 (m, $1 \mathrm{H}), 1.94-1.77$ (m, $2 \mathrm{H}), 1.31$ (s, $3 \mathrm{H}) ;{ }^{13} \mathrm{C} \mathrm{NMR}\left(150 \mathrm{MHz}, \mathrm{CDCl}_{3}\right): \delta$ 169.3, 150.4, 142.3, 110.4, 108.6, 67.4, 58.7, 43.1, 33.8, 28.5, 27.2; IR (neat): 3324, 2972, 2931, 1621, 1414 , 1378, 1220, 1085, $878 \mathrm{~cm}^{-1}$; HRMS (ESI) $\mathrm{m} / z$ calcd for $\mathrm{C}_{11} \mathrm{H}_{16} \mathrm{NO}_{3}\left[(\mathrm{M}+\mathrm{H})^{+}\right] 210.1130$, found 210.1134 .

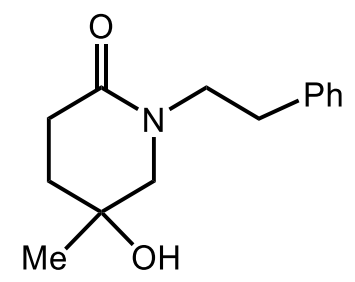

5-hydroxy-5-methyl-1-phenethylpiperidin-2-one (8): This compound was prepared according to the General Procedure using 4-methylpent-4-enoic acid (57 mg, $0.5 \mathrm{mmol})$, 2-phenylethan-1- 
amine $(182 \mathrm{mg}, 1.5 \mathrm{mmol})$. After purification by column chromatography $\mathrm{SiO}_{2}(50 \%$ EtOAc/Hexane to $5 \% \mathrm{MeOH} / \mathrm{EtOAc}$ ), the title compound was isolated as a colorless oil (82 $\mathrm{mg}$, $70 \%$ yield).

${ }^{1} \mathrm{H}$ NMR (600 MHz, $\left.\mathrm{CDCl}_{3}\right): \delta$ 7.27-7.21 (m, $\left.2 \mathrm{H}\right)$, 7.19-7.15 (m, $\left.3 \mathrm{H}\right), 3.58-3.49$ (m, $\left.1 \mathrm{H}\right), 3.49-$ $3.39(\mathrm{~m}, 1 \mathrm{H}), 3.09$ (d, $J=12.5 \mathrm{~Hz}, 1 \mathrm{H}), 3.03(\mathrm{~d}, J=12.5 \mathrm{~Hz}, 1 \mathrm{H}), 2.88-2.74(\mathrm{~m}, 2 \mathrm{H}), 2.60-2.53$ (m, $1 \mathrm{H}), 2.35-2.24(\mathrm{~m}, 1 \mathrm{H}), 1.85-1.75(\mathrm{~m}, 1 \mathrm{H}), 1.73-1.66(\mathrm{~m}, 1 \mathrm{H}), 1.22(\mathrm{~s}, 3 \mathrm{H}) ;{ }^{13} \mathrm{C}$ NMR $(150$ $\left.\mathrm{MHz}, \mathrm{CDCl}_{3}\right): \delta 169.4,138.9,128.8,128.4,126.3,66.9,59.7,49.2,33.6,33.2,28.5,27.2 ; \mathrm{IR}$ (neat): 3269, 2974, 2933, 2860, 1602, 1499, 1275, 926, 700, $498 \mathrm{~cm}^{-1}$; HRMS (ESI) $\mathrm{m} / z$ calcd for $\mathrm{C}_{14} \mathrm{H}_{20} \mathrm{NO}_{2}\left[(\mathrm{M}+\mathrm{H})^{+}\right]$234.1494, found 234.1498.

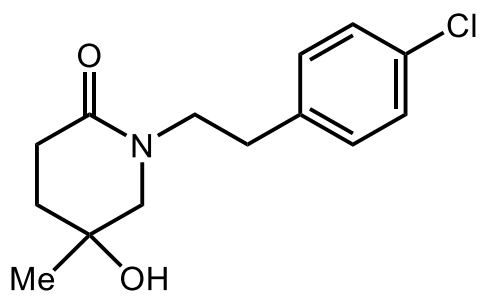

1-(4-chlorophenethyl)-5-hydroxy-5-methylpiperidin-2-one (9): This compound was prepared according to the General Procedure using 4-methylpent-4-enoic acid (57 mg, $0.5 \mathrm{mmol}), 2-(4-$ chlorophenyl)ethan-1-amine (233 mg $1.5 \mathrm{mmol})$. After purification by column chromatography $\mathrm{SiO}_{2}(50 \% \mathrm{EtOAc} / \mathrm{Hexane}$ to $5 \% \mathrm{MeOH} / \mathrm{EtOAc})$, the title compound was isolated as a white solid (118 $\mathrm{mg}, 88 \%$ yield).

${ }^{1} \mathrm{H} \mathrm{NMR}\left(600 \mathrm{MHz}, \mathrm{CDCl}_{3}\right) \delta 7.25(\mathrm{~d}, J=8.3 \mathrm{~Hz}, 2 \mathrm{H}), 7.15(\mathrm{~d}, J=8.3 \mathrm{~Hz}, 2 \mathrm{H}), 3.45-3.56(\mathrm{~m}$, $2 \mathrm{H}), 3.17(\mathrm{~d}, J=12.5 \mathrm{~Hz}, 1 \mathrm{H}), 3.08(\mathrm{dd}, J=12.6,1.8 \mathrm{~Hz}, 1 \mathrm{H}), 2.88-2.77(\mathrm{~m}, 2 \mathrm{H}), 2.64-2.55$ (m, $1 \mathrm{H}), 2.39-2.32(\mathrm{~m}, 1 \mathrm{H}), 1.87-181(\mathrm{~m}, 1 \mathrm{H}), 1.80-173(\mathrm{~m}, 1 \mathrm{H}), 1.29(\mathrm{~s}, 3 \mathrm{H}) ;{ }^{13} \mathrm{C}$ NMR $(150$ $\left.\mathrm{MHz}, \mathrm{CDCl}_{3}\right) \delta 169.4,137.3,132.1,130.2,128.6,67.2,59.7,48.9,33.8,32.6,28.5,27.3 ; \mathrm{IR}$ (neat): 
3281, 2963, 2934, 1609, 1499, 1485, 1402, 1281, 1244, 1169, 1126, 1009 cm-1; HRMS (ESI) m/z calcd for $\mathrm{C}_{14} \mathrm{H}_{19} \mathrm{ClNO}_{2}[(\mathrm{M}+\mathrm{H})+] 268.1104$, found 268.1102 .<smiles>CC1(O)CCC(=O)N(CCc2cccs2)C1</smiles>

5-hydroxy-5-methyl-1-(2-(thiophen-2-yl)ethyl)piperidin-2-one (10): This compound was prepared according to the General Procedure using 4-methylpent-4-enoic acid (57 mg, $0.5 \mathrm{mmol}$ ), 2-thiopheneethylamine (191 mg, $1.5 \mathrm{mmol}$ ). After purification by column chromatography $\mathrm{SiO}_{2}$ (100\% EtOAc to $5 \% \mathrm{MeOH} / \mathrm{EtOAc}$ ), the title compound was isolated as a colorless oil (89 $\mathrm{mg}$, $74 \%$ yield).

${ }^{1} \mathrm{H}$ NMR $\left(600 \mathrm{MHz}, \mathrm{CDCl}_{3}\right): \delta$ 7.16-7.09 (m, $\left.1 \mathrm{H}\right), 6.94-6.88(\mathrm{~m}, 1 \mathrm{H}), 6.86-6.80(\mathrm{~m}, 1 \mathrm{H}), 3.67-$ 3.57 (m, 1 H), 3.54-3.434 (m, 1 H), 3.28 (br s, 1 H), 3.19-3.00 (m, 4 H), 2.65-2.54 (m, 1 H), 2.39$2.29(\mathrm{~m}, 1 \mathrm{H}), 1.87-1.79(\mathrm{~m}, 1 \mathrm{H}), 1.79-1.69(\mathrm{~m}, 1 \mathrm{H}), 1.26(\mathrm{~s}, 3 \mathrm{H}) ;{ }^{13} \mathrm{C} \mathrm{NMR}\left(150 \mathrm{MHz}, \mathrm{CDCl}_{3}\right)$ : $\delta 169.7,141.1,126.9,125.3,123.7,66.8,59.9,49.3,33.5,28.5,27.2,27.2 ;$ IR (neat): 3285,3110 , 2964, 2930, 2858, 1603, 1499, 1245, 1126, 925, $715 \mathrm{~cm}^{-1}$; HRMS (ESI) $\mathrm{m} / \mathrm{z}$ calcd for $\mathrm{C}_{12} \mathrm{H}_{18} \mathrm{NO}_{2} \mathrm{~S}$ $[(\mathrm{M}+\mathrm{H})+] 240.1058$, found 240.1060 .

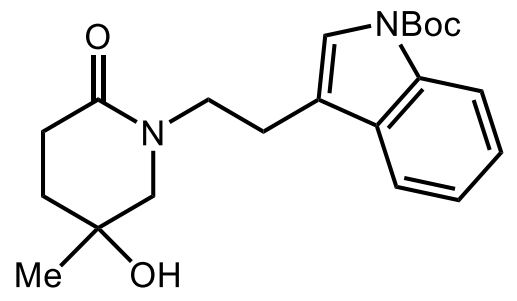

tert-butyl 3-(2-(5-hydroxy-5-methyl-2-oxopiperidin-1-yl)ethyl)-1H-indole-1-carboxylate (11): This compound was prepared according to the General Procedure using 4-methylpent-4enoic acid (57 mg, $0.5 \mathrm{mmol}$ ), tert-butyl 3-(2-aminoethyl)-1H-indole-1-carboxylate (391 mg, 1.5 
mmol). After purification by column chromatography $\mathrm{SiO}_{2}$ (80\% EtOAc in Hexanes to EtOAc), the title compound was isolated as a colorless oil (123 mg, 66\% yield).

${ }^{1} \mathrm{H}$ NMR (600 MHz, $\mathrm{CDCl}_{3}$ ): $\delta 8.09$ (br s, $\left.1 \mathrm{H}\right), 7.58$ (d, J= 7.6 Hz, $\left.1 \mathrm{H}\right), 7.39$ (br s, $\left.1 \mathrm{H}\right), 7.32-$ $7.25(\mathrm{~m}, 1 \mathrm{H}), 7.25-7.18(\mathrm{~m}, 1 \mathrm{H}), 3.61-3.54(\mathrm{~m}, 2 \mathrm{H}), 3.21(\mathrm{~d}, J=12.5 \mathrm{~Hz}, 1 \mathrm{H}), 3.11(\mathrm{~d}, J=12.5$ Hz, $1 \mathrm{H}), 2.97-2.89$ (m, 2 H), 2.65-2.56 (m, $1 \mathrm{H}), 2.42-2.33$ (m, $1 \mathrm{H}), 1.86-1.71$ (m, $2 \mathrm{H}), 1.63$ (s, $9 \mathrm{H}), 1.25(\mathrm{~s}, 3 \mathrm{H}) ;{ }^{13} \mathrm{C} \mathrm{NMR}\left(150 \mathrm{MHz}, \mathrm{CDCl}_{3}\right): \delta 169.4,149.7,135.4,130.5,124.4,123.1,122.5$, 118.9, 117.7, 115.3, 83.5, 67.3, 59.9, 47.9, 33.8, 28.6, 28.2, 27.2, 22.6; IR (neat): 3251, 2979, 2929, $2856,1726,1605,1374,1152,1076,746 \mathrm{~cm}^{-1}$; HRMS (ESI) m/z calcd for $\mathrm{C}_{21} \mathrm{H}_{29} \mathrm{~N}_{2} \mathrm{O}_{4}[(\mathrm{M}+\mathrm{H})+]$ 373.2127, found 373.2131 .

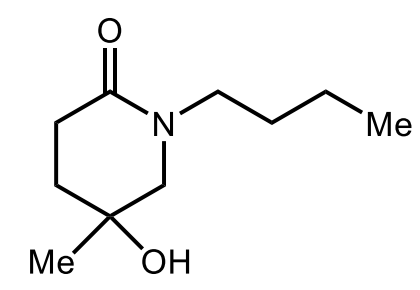

1-butyl-5-hydroxy-5-methylpiperidin-2-one (12): This compound was prepared according to the General Procedure using 4-methylpent-4-enoic acid (57 mg, $0.5 \mathrm{mmol})$, butylamine (110 mg, 1.5 mmol). After purification by column chromatography $\mathrm{SiO}_{2}(100 \%$ EtOAc to $5 \% \mathrm{MeOH} / \mathrm{EtOAc})$, the title compound was isolated as a colorless oil (63 mg, $68 \%$ yield).

${ }^{1} \mathrm{H}$ NMR (600 MHz, $\left.\mathrm{CDCl}_{3}\right): \delta 3.41-3.34(\mathrm{~m}, 1 \mathrm{H}), 3.26-3.18(\mathrm{~m}, 2 \mathrm{H}), 3.15(\mathrm{~d}, J=12.7 \mathrm{~Hz}, 1 \mathrm{H})$, 2.61-2.52 (m, $1 \mathrm{H}), 2.35-2.27$ (m, $1 \mathrm{H}), 1.88-1.82(\mathrm{~m}, 1 \mathrm{H}), 1.79-1.71(\mathrm{~m}, 1 \mathrm{H}), 1.53-1.44(\mathrm{~m}, 2 \mathrm{H})$, $1.31(\mathrm{~s}, 3 \mathrm{H}), 1.28(\mathrm{q}, J=7.6 \mathrm{~Hz}, 2 \mathrm{H}), 0.89(\mathrm{t}, J=7.6 \mathrm{~Hz}, 3 \mathrm{H}) ;{ }^{13} \mathrm{C} \mathrm{NMR}\left(150 \mathrm{MHz}, \mathrm{CDCl}_{3}\right)$ : $\delta 169.3,66.9,58.9,46.9,33.7,28.9,28.5,27.1,20.0,13.8$; IR (neat): 3359, 2958, 2930, 2872, 1611, 1499, 1281, 924, $638 \mathrm{~cm}^{-1}$; HRMS (ESI) m/z calcd for $\mathrm{C}_{10} \mathrm{H}_{19} \mathrm{NO}_{2}[(\mathrm{M}+\mathrm{H})+] 185.1416$, found 185.1419 . 


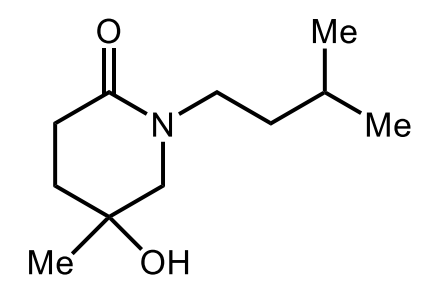

5-hydroxy-1-isopentyl-5-methylpiperidin-2-one (13): This compound was prepared according to the General Procedure using 4-methylpent-4-enoic acid ( $57 \mathrm{mg}, 0.5 \mathrm{mmol})$, isopentylamine (131 $\mathrm{mg}, 1.5 \mathrm{mmol})$. After purification by column chromatography $\mathrm{SiO}_{2}(100 \%$ EtOAc to $5 \%$ $\mathrm{MeOH} / \mathrm{EtOAc}$ ), the title compound was isolated as a colorless oil (61 mg, $61 \%$ yield).

${ }^{1} \mathrm{H}$ NMR (600 MHz, CDCl $): \delta$ 3.44-3.35 (m, $\left.1 \mathrm{H}\right), 3.29-3.19(\mathrm{~m}, 2 \mathrm{H}), 3.15(\mathrm{~d}, J=12.5 \mathrm{~Hz}, 1 \mathrm{H})$, 2.62-2.51 (m, $1 \mathrm{H}), 2.37-2.27(\mathrm{~m}, 1 \mathrm{H}), 1.89-1.81(\mathrm{~m}, 1 \mathrm{H}), 1.80-1.72(\mathrm{~m}, 1 \mathrm{H}), 1.60-1.49(\mathrm{~m}, 1$ H), 1.43-1.35 (m, $2 \mathrm{H}), 1.32(\mathrm{~s}, 3 \mathrm{H}), 0.90(\mathrm{~d}, J=6.6 \mathrm{~Hz}, 3 \mathrm{H}), 0.89(\mathrm{~d}, J=6.6 \mathrm{~Hz}, 3 \mathrm{H}) ;{ }^{13} \mathrm{C} \mathrm{NMR}$ (150 MHz, $\left.\mathrm{CDCl}_{3}\right): \delta$ 169.1, 66.9, 58.8, 45.6, 35.5, 33.8, 28.6, 27.1, 25.9, 22.5, 22.5; IR (neat): 3278, 2952, 2927, 2867, 1609, 1509, 1366, 1209, $876 \mathrm{~cm}^{-1}$; H HRMS (ESI) m/z calcd for $\mathrm{C}_{11} \mathrm{H}_{22} \mathrm{NO}_{2}[(\mathrm{M}+\mathrm{H})+] 200.1651$, found 200.1648 .

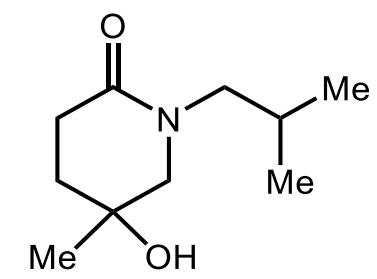

5-hydroxy-1-isobutyl-5-methylpiperidin-2-one (14): This compound was prepared according to the General Procedure using 4-methylpent-4-enoic acid (57 mg, $0.5 \mathrm{mmol})$, 2-methylpropan-1amine $(110 \mathrm{mg} 1.5 \mathrm{mmol})$. After purification by column chromatography $\mathrm{SiO}_{2}(50 \%$ EtOAc/Hexane to $5 \% \mathrm{MeOH} / \mathrm{EtOAc})$, the title compound was isolated as a white solid (67 mg, $72 \%$ yield). 
${ }^{1} \mathrm{H}$ NMR (600 MHz, CDCl $) \delta 3.33(\mathrm{br} \mathrm{s}, 1 \mathrm{H}), 3.23-3.13(\mathrm{~m}, 3 \mathrm{H}), 3.08(\mathrm{dd}, J=13.2,7.2 \mathrm{~Hz}, 1$ H), 2.63-2.54 (m, $1 \mathrm{H}), 2.36-2.29(\mathrm{~m}, 1 \mathrm{H}), 1.98-1.89(\mathrm{~m}, 1 \mathrm{H}), 1.88-1.81(\mathrm{~m}, 1 \mathrm{H}), 1.80-1.73(\mathrm{~m}$, $1 \mathrm{H}), 1.31(\mathrm{~s}, 3 \mathrm{H}), 0.87(\mathrm{~d}, J=6.6 \mathrm{~Hz}, 3 \mathrm{H}), 0.85(\mathrm{~d}, J=6.6 \mathrm{~Hz}, 3 \mathrm{H}),{ }^{13} \mathrm{C} \mathrm{NMR}\left(150 \mathrm{MHz}, \mathrm{CDCl}_{3}\right)$ $\delta 169.6,66.9,59.4,54.2,33.8,28.6,27.2,26.2,20.0,19.9$; IR (neat): 3254, 2964, 2923, 2868 , $1608,1500,1461,1367,1285,1127 \mathrm{~cm}-1$; HRMS (ESI) m/z calcd for $\mathrm{C}_{10} \mathrm{H}_{20} \mathrm{NO}_{2}[(\mathrm{M}+\mathrm{H})+]$ 186.1494, found 186.1490 .

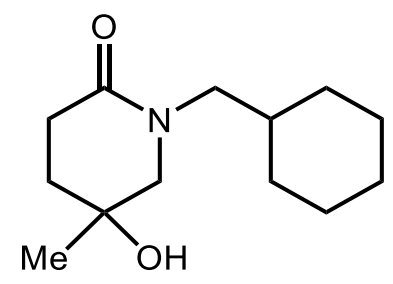

1-(cyclohexylmethyl)-5-hydroxy-5-methylpiperidin-2-one (15): This compound was prepared according to the General Procedure using 4-methylpent-4-enoic acid (57 mg, $0.5 \mathrm{mmol}$ ), cyclohexylmethanamine $(170 \mathrm{mg}, 1.5 \mathrm{mmol})$ in methanol $(3.5 \mathrm{~mL})$ for $36 \mathrm{~h}$. After purification by column chromatography $\mathrm{SiO}_{2}(50 \% \mathrm{EtOAc} / \mathrm{Hexane}$ to $5 \% \mathrm{MeOH} / \mathrm{EtOAc})$, the title compound was isolated as a brown solid ( $80 \mathrm{mg}, 71 \%$ yield).

${ }^{1} \mathrm{H}$ NMR (600 MHz, $\left.\mathrm{CDCl}_{3}\right): \delta 3.30-3.19(\mathrm{~m}, 2 \mathrm{H}), 3.18-3.12(\mathrm{~m}, 1 \mathrm{H}), 3.07(\mathrm{dd}, J=13.4,6.8 \mathrm{~Hz}$, $1 \mathrm{H}), 2.58(\mathrm{ddd}, J=17.7,10.4,7.1 \mathrm{~Hz}, 1 \mathrm{H}), 2.40-2.25(\mathrm{~m}, 1 \mathrm{H}), 1.91-1.80(\mathrm{~m}, 1 \mathrm{H}), 1.80-1.73$ (m, $1 \mathrm{H}), 1.67-1.56(\mathrm{~m}, 6 \mathrm{H}), 1.32(\mathrm{~s}, 3 \mathrm{H}), 1.26-1.06(\mathrm{~m}, 3 \mathrm{H}), 0.95-0.83(\mathrm{~m}, 2 \mathrm{H}) ;{ }^{13} \mathrm{C}$ NMR $(150$ $\left.\mathrm{MHz}, \mathrm{CDCl}_{3}\right): \delta 169.6,67.1,59.7,53.3,35.6,33.8,30.7,30.6,28.6,27.2,26.3,25.8$; IR (neat): 3229, 2917, 2841, 1606, 1507, 1281, 1130, 923, $544 \mathrm{~cm}^{-1}$; HRMS (ESI) m/z calcd for $\mathrm{C}_{13} \mathrm{H}_{24} \mathrm{NO}_{2}$ $[(\mathrm{M}+\mathrm{H})+]$ 226.1807, found 226.1808. 


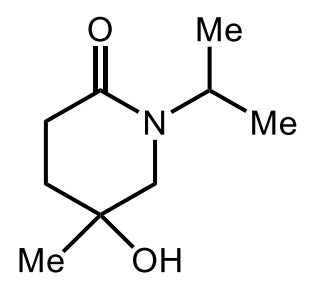

5-hydroxy-1-isopropyl-5-methylpiperidin-2-one (16): This compound was prepared according to the General Procedure using 4-methylpent-4-enoic acid (57 mg, $0.5 \mathrm{mmol}$ ), propan-2-amine (89 mg $1.5 \mathrm{mmol}$ ). After purification by column chromatography $\mathrm{SiO}_{2}(50 \%$ EtOAc/Hexane to $5 \%$ $\mathrm{MeOH} / \mathrm{EtOAc}$ ), the title compound was isolated as a white solid (36 mg, $42 \%$ yield).

${ }^{1} \mathrm{H}$ NMR $\left(600 \mathrm{MHz}, \mathrm{CDCl}_{3}\right) \delta$ 4.95-4.87 (m, $\left.1 \mathrm{H}\right), 3.09(\mathrm{~s}, 2 \mathrm{H}), 2.63-2.54(\mathrm{~m}, 1 \mathrm{H}), 2.42-2.35(\mathrm{~m}$, $1 \mathrm{H}), 2.16$ (br s, 1H), 1.88-1.75 (m, $2 \mathrm{H}), 1.35$ (s, $3 \mathrm{H}), 1.09$ (d, $J=7.2 \mathrm{~Hz}, 3 \mathrm{H}), 1.08$ (d, $J=7.2$ $\mathrm{Hz}, 3 \mathrm{H}) ;{ }^{13} \mathrm{C} \mathrm{NMR}\left(150 \mathrm{MHz}, \mathrm{CDCl}_{3}\right) \delta$ 168.6, 67.3, 51.7, 43.4, 33.7, 29.0, 27.0, 19.1, 19.0; IR (neat): 3333, 2969, 2933, 1600, 1489, 1460, 1303, 1282, 1210, $1030 \mathrm{~cm}^{-1}$; HRMS (ESI) m/z calcd for $\mathrm{C}_{9} \mathrm{H}_{18} \mathrm{NO}_{2}\left[(\mathrm{M}+\mathrm{H})^{+}\right]$172.1338, found 172.1395.

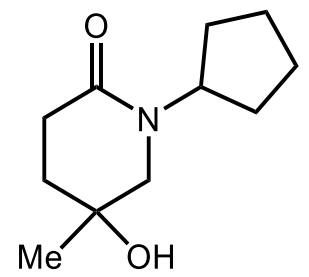

1-cyclopentyl-5-hydroxy-5-methylpiperidin-2-one (17): This compound was prepared according to the General Procedure using 4-methylpent-4-enoic acid (57 mg, $0.5 \mathrm{mmol}$ ), cyclopentanamine (128 mg, $1.5 \mathrm{mmol}$ ) for 36 hours. After purification by column chromatography $\mathrm{SiO}_{2}(100 \%$ EtOAc to $5 \% \mathrm{MeOH} / \mathrm{EtOAc})$, the title compound was isolated as a colorless oil (47 $\mathrm{mg}, 48 \%$ yield).

${ }^{1} \mathrm{H}$ NMR (600 MHz, $\left.\mathrm{CDCl}_{3}\right): \delta$ 5.03-4.94 (m, $\left.1 \mathrm{H}\right), 3.15-3.06$ (m, $\left.2 \mathrm{H}\right), 2.97$ (br s, $\left.1 \mathrm{H}\right), 2.63-2.52$ (m, $1 \mathrm{H}), 2.43-2.31(\mathrm{~m}, 1 \mathrm{H}), 1.89-1.71(\mathrm{~m}, 4 \mathrm{H}), 1.70-1.51(\mathrm{~m}, 4 \mathrm{H}), 1.50-1.40(\mathrm{~m}, 2 \mathrm{H}), 1.33(\mathrm{~s}$, 
$3 \mathrm{H}) ;{ }^{13} \mathrm{C} \mathrm{NMR}\left(150 \mathrm{MHz}, \mathrm{CDCl}_{3}\right): \delta 169.5,67.1,53.7,52.8,33.6,29.1,27.7,27.7,27.0,24.3$, 24.1; IR (neat): 3256, 2956, 2867, 1606, 1500, 1280, 1243, 1126, $928 \mathrm{~cm}^{-1}$; HRMS (ESI) m/z calcd for $\mathrm{C}_{11} \mathrm{H}_{20} \mathrm{NO}_{2}\left[(\mathrm{M}+\mathrm{H})^{+}\right]$198.1494, found 198.1496.

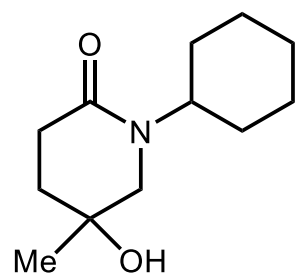

1-(cyclohexylmethyl)-5-hydroxy-5-methylpiperidin-2-one (18): This compound was prepared according to the General Procedure using 4-methylpent-4-enoic acid (57 mg, $0.5 \mathrm{mmol}$ ), cyclohexylamine ( $149 \mathrm{mg}, 1.5 \mathrm{mmol})$ in methanol $(3.5 \mathrm{~mL})$ for $36 \mathrm{~h}$. After purification by column chromatography $\mathrm{SiO}_{2}(50 \% \mathrm{EtOAc} / \mathrm{Hexane}$ to $5 \% \mathrm{MeOH} / \mathrm{EtOAc})$, the title compound was isolated as a brown solid (63 $\mathrm{mg}, 60 \%$ yield).

${ }^{1} \mathrm{H}$ NMR (600 MHz, $\left.\mathrm{CDCl}_{3}\right): \delta$ 4.47-4.36 (m, $\left.1 \mathrm{H}\right), 3.15-3.02(\mathrm{~m}, 2 \mathrm{H}), 2.89$ (br s, $\left.1 \mathrm{H}\right), 2.61-2.50$ (m, 1 H), 2.39-2.30 (m, 1 H), 1.85-1.69 (m, 4 H), 1.66-1.55 (m, 3 H), 1.41-1.25 (m, 7 H), 1.08$0.97(\mathrm{~m}, 1 \mathrm{H}) ;{ }^{13} \mathrm{C} \mathrm{NMR}\left(150 \mathrm{MHz}, \mathrm{CDCl}_{3}\right): \delta 168.9,67.1,52.9,51.9,33.5,29.5,29.2,29.1,27.0$, 25.6, 25.5, 25.5; IR (neat): 3269, 2927, 2850, 1596, 1489, 1286, 1245, 695, $558 \mathrm{~cm}^{-1}$; HRMS (ESI) $\mathrm{m} / \mathrm{z}$ calcd for $\mathrm{C}_{12} \mathrm{H}_{22} \mathrm{NO}_{2}\left[(\mathrm{M}+\mathrm{H})^{+}\right] 212.1651$, found 212.1645.

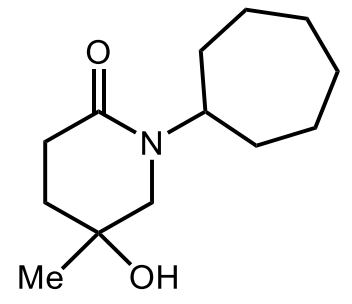

1-(cyclohexylmethyl)-5-hydroxy-5-methylpiperidin-2-one (19): This compound was prepared according to the General Procedure using 4-methylpent-4-enoic acid $(57 \mathrm{mg}, 0.5 \mathrm{mmol}$ ), cycloheptylamine (173 mg, $1.5 \mathrm{mmol})$ in methanol $(3.5 \mathrm{~mL})$ for $36 \mathrm{~h}$. After purification by column 
chromatography $\mathrm{SiO}_{2}(50 \% \mathrm{EtOAc} / \mathrm{Hexane}$ to $5 \% \mathrm{MeOH} / \mathrm{EtOAc})$, the title compound was isolated as a colorless oil (68 $\mathrm{mg}, 60 \%$ yield).

${ }^{1} \mathrm{H}$ NMR (600 MHz, $\left.\mathrm{CDCl}_{3}\right): \delta$ 4.62-4.52 (m, $\left.1 \mathrm{H}\right), 3.15-3.06(\mathrm{~m}, 2 \mathrm{H}), 2.92(\mathrm{br} \mathrm{s}, 1 \mathrm{H}), 2.59-2.48$ (m, $1 \mathrm{H}), 2.35-2.25(\mathrm{~m}, 1 \mathrm{H}), 1.83-1.76(\mathrm{~m}, 1 \mathrm{H}), 1.75-1.57(\mathrm{~m}, 7 \mathrm{H}), 1.55-1.39(\mathrm{~m}, 6 \mathrm{H}), 1.31(\mathrm{~s}$ $3 \mathrm{H}) ;{ }^{13} \mathrm{C}$ NMR $\left(150 \mathrm{MHz}, \mathrm{CDCl}_{3}\right): \delta 168.3,67.0,53.8,53.0,33.6,31.7,31.7,29.0,27.6,27.5$, 26.9, 25.1, 25.0; IR (neat): 3275, 2920, 2854, 1592, 1491, 1458, 1302, 930, $510 \mathrm{~cm}^{-1}$; HRMS (ESI) $\mathrm{m} / \mathrm{z}$ calcd for $\mathrm{C}_{13} \mathrm{H}_{24} \mathrm{NO}_{2}\left[(\mathrm{M}+\mathrm{H})^{+}\right] 226.1807$, found 226.1805 .

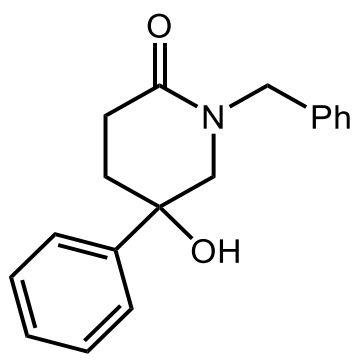

1-benzyl-5-hydroxy-5-phenylpiperidin-2-one (20): This compound was prepared according to the General Procedure using 4-phenylpent-4-enoic acid ( $88 \mathrm{mg}, 0.5 \mathrm{mmol})$, benzylamine ( $161 \mathrm{mg}$, $1.5 \mathrm{mmol})$ for 36 hours. After purification by column chromatography $\mathrm{SiO}_{2}(100 \%$ EtOAc to $5 \%$ $\mathrm{MeOH} / \mathrm{EtOAc}$ ), the title compound was isolated as a colorless oil (101 mg, 70\% yield).

${ }^{1} \mathrm{H}$ NMR $\left(600 \mathrm{MHz}, \mathrm{CDCl}_{3}\right): \delta$ 7.42-7.37 (m, $\left.2 \mathrm{H}\right), 7.32-7.19(\mathrm{~m}, 8 \mathrm{H}), 4.64(\mathrm{~d}, J=14.9 \mathrm{~Hz}, 1 \mathrm{H})$, $4.46(\mathrm{~d}, J=14.9 \mathrm{~Hz}, 1 \mathrm{H}), 3.46$ (d, $J=12.9 \mathrm{~Hz}, 1 \mathrm{H}), 3.27-3.16(\mathrm{~m}, 2 \mathrm{H}), 2.84-2.76(\mathrm{~m}, 1 \mathrm{H}), 2.49-$ $2.42(\mathrm{~m}, 1 \mathrm{H}), 2.36-2.28(\mathrm{~m}, 1 \mathrm{H}), 2.07-1.99(\mathrm{~m}, 1 \mathrm{H}) ;{ }^{13} \mathrm{C} \mathrm{NMR}\left(150 \mathrm{MHz}, \mathrm{CDCl}_{3}\right): \delta 169.4$ 144.5, 136.6, 128.6, 128.4, 128.0, 127.7, 127.4, 124.8, 70.7, 58.6, 50.2, 33.0, 28.6; IR (neat): 3318, $3058,3026,2931,1614,1502,1415,1232,1018,704 \mathrm{~cm}^{-1}$; HRMS (ESI) m/z calcd for $\mathrm{C}_{18} \mathrm{H}_{20} \mathrm{NO}_{2}$ $\left[(\mathrm{M}+\mathrm{H})^{+}\right] 282.1494$, found 282.1494 


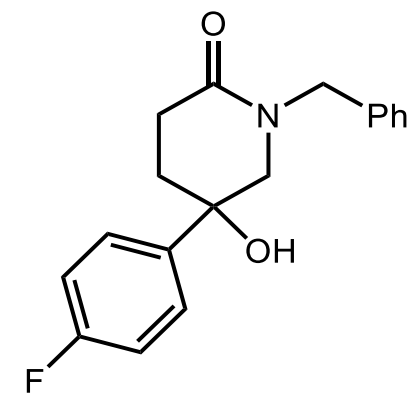

1-benzyl-5-(4-fluorophenyl)-5-hydroxypiperidin-2-one (21): This compound was prepared according to the General Procedure using 4-(4-fluorophenyl)pent-4-enoic acid (97 mg, $0.5 \mathrm{mmol}$ ), benzylamine (161 mg, $1.5 \mathrm{mmol}$ ) for 36 hours. After purification by column chromatography $\mathrm{SiO}_{2}$ (100\% EtOAc to $5 \% \mathrm{MeOH} / \mathrm{EtOAc}$ ), the title compound was isolated as a colorless oil (108 mg, $72 \%$ yield).

${ }^{1} \mathrm{H}$ NMR (600 MHz, $\left.\mathrm{CDCl}_{3}\right): \delta$ 7.38-7.33 (m, $\left.2 \mathrm{H}\right)$, 7.30-7.18 (m, $\left.5 \mathrm{H}\right), 6.98-6.92(\mathrm{~m}, 2 \mathrm{H}), 4.66$ $(\mathrm{d}, J=14.9 \mathrm{~Hz}, 1 \mathrm{H}), 4.40(\mathrm{~d}, J=14.9 \mathrm{~Hz}, 1 \mathrm{H}), 3.59(\mathrm{br} \mathrm{s}, 1 \mathrm{H}), 3.41(\mathrm{~d}, J=12.9 \mathrm{~Hz}, 1 \mathrm{H}), 3.25-$ $3.20(\mathrm{~m}, 1 \mathrm{H}), 2.82-2.74(\mathrm{~m}, 1 \mathrm{H}), 2.47-2.40(\mathrm{~m}, 1 \mathrm{H}), 2.33-2.25(\mathrm{~m}, 1 \mathrm{H}), 2.05-1.99(\mathrm{~m}, 1 \mathrm{H}) ;{ }^{13} \mathrm{C}$ NMR (150 MHz, $\left.\mathrm{CDCl}_{3}\right): \delta 169.4,162.6\left(\mathrm{~d}, J_{\mathrm{C}, \mathrm{F}}=246.0 \mathrm{~Hz}\right), 140.3\left(\mathrm{~d}, J_{\mathrm{C}, \mathrm{F}}=3.5 \mathrm{~Hz}\right), 136.5$, 128.3, 128.0, 127.5, $126.7\left(\mathrm{~d}, J_{\mathrm{C}, \mathrm{F}}=8.0 \mathrm{~Hz}\right), 115.2\left(\mathrm{~d}, J_{\mathrm{C}, \mathrm{F}}=21.3 \mathrm{~Hz}\right), 70.4,58.6,50.2,33.0,28.6$; IR (neat): 3264, 3065, 3033, 2926, 2863, 1608, 1508, 1228, 834, $698 \mathrm{~cm}^{-1}$; HRMS (ESI) m/z calcd for $\mathrm{C}_{18} \mathrm{H}_{19} \mathrm{FNO}_{2}\left[(\mathrm{M}+\mathrm{H})^{+}\right]$300.1400, found 300.1411.<smiles>O=C1CCC(O)(c2ccc(Cl)cc2)CN1Cc1ccccc1</smiles> 
1-benzyl-5-(4-chlorophenyl)-5-hydroxypiperidin-2-one (22): This compound was prepared according to the General Procedure using 4-(4-chlorophenyl)pent-4-enoic acid (105 mg, $0.5 \mathrm{mmol}$ ), benzylamine (161 mg, $1.5 \mathrm{mmol}$ ) for 36 hours. After purification by column chromatography $\mathrm{SiO}_{2}$ $(100 \%$ EtOAc to $5 \% \mathrm{MeOH} / \mathrm{EtOAc})$, the title compound was isolated as a colorless oil (95mg, $60 \%$ yield).

${ }^{1} \mathrm{H}$ NMR (600 MHz, $\left.\mathrm{CDCl}_{3}\right): \delta 7.35-7.15(\mathrm{~m}, 10 \mathrm{H}), 4.64(\mathrm{~d}, J=14.9 \mathrm{~Hz}, 1 \mathrm{H}), 4.42(\mathrm{~d}, J=14.9$ Hz, $1 \mathrm{H}), 3.42(\mathrm{~d}, J=12.9 \mathrm{~Hz}, 1 \mathrm{H}), 3.38$ (br s, $1 \mathrm{H}), 3.21(\mathrm{dd}, J=12.9,1.3 \mathrm{~Hz}, 1 \mathrm{H}), 2.82-2.73$ (m, $1 \mathrm{H}), 2.46-2.39(\mathrm{~m}, 1 \mathrm{H}), 2.30-2.22(\mathrm{~m}, 1 \mathrm{H}), 2.04-1.98(\mathrm{~m}, 1 \mathrm{H}) ;{ }^{13} \mathrm{C} \mathrm{NMR}\left(150 \mathrm{MHz}, \mathrm{CDCl}_{3}\right)$ : $\delta 169.4,143.1,136.4,133.5,128.6,128.5,128.0,127.5,126.4,70.4,58.5,50.2,32.9,28.5 ;$ IR (neat): 3354, 3088, 3060, 2927, 1613, 1488, 1231, 1099, 1012, 824, $488 \mathrm{~cm}^{-1} ; \mathrm{HRMS}(\mathrm{ESI}) \mathrm{m} / \mathrm{z}$ calcd for $\mathrm{C}_{18} \mathrm{H}_{19} \mathrm{ClNO}_{2}\left[(\mathrm{M}+\mathrm{H})^{+}\right] 316.1104$, found 316.1178 .

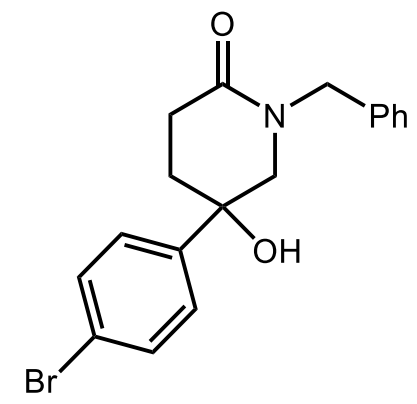

1-benzyl-5-(4-bromophenyl)-5-hydroxypiperidin-2-one (23): This compound was prepared according to the General Procedure using 4-(4-bromophenyl)pent-4-enoic acid (128 mg, 0.5 mmol), benzylamine (161 $\mathrm{mg}, 1.5 \mathrm{mmol})$ for 36 hours. After purification by column chromatography $\mathrm{SiO}_{2}(100 \%$ EtOAc to $5 \% \mathrm{MeOH} / \mathrm{EtOAc})$, the title compound was isolated as a colorless oil (65 mg, 36\% yield).

${ }^{1} \mathrm{H}$ NMR (600 MHz, $\left.\mathrm{CDCl}_{3}\right): \delta$ 7.41-7.35 (m, $\left.2 \mathrm{H}\right), 7.31-7.16(\mathrm{~m}, 7 \mathrm{H}), 4.65(\mathrm{~d}, J=14.6 \mathrm{~Hz}, 1 \mathrm{H})$, $4.39(\mathrm{~d}, J=14.6 \mathrm{~Hz}, 1 \mathrm{H}), 3.54(\mathrm{br} \mathrm{s}, 1 \mathrm{H}), 3.41(\mathrm{~d}, J=12.9 \mathrm{~Hz}, 1 \mathrm{H}), 3.21(\mathrm{~d}, J=12.9 \mathrm{~Hz}, 1 \mathrm{H})$, 
2.82-2.72 (m, $1 \mathrm{H}), 2.47-2.37(\mathrm{~m}, 1 \mathrm{H}), 2.31-2.20(\mathrm{~m}, 1 \mathrm{H}), 2.04-1.97$ (m, $1 \mathrm{H}) ;{ }^{13} \mathrm{C}$ NMR $(150$ $\left.\mathrm{MHz}, \mathrm{CDCl}_{3}\right): \delta 169.4,143.7,136.4,131.5,128.6,128.0,127.5,126.7,121.7,70.4,58.4,50.3$, 32.8, 28.5; IR (neat): 3288, 3060, 3028, 2924, 1613, 1487, 1231, 1007, 821, $703 \mathrm{~cm}^{-1}$; HRMS (ESI) $\mathrm{m} / \mathrm{z}$ calcd for $\mathrm{C}_{18} \mathrm{H}_{19} \mathrm{BrNO}_{2}\left[(\mathrm{M}+\mathrm{H})^{+}\right] 360.0599$, found 360.0613 .

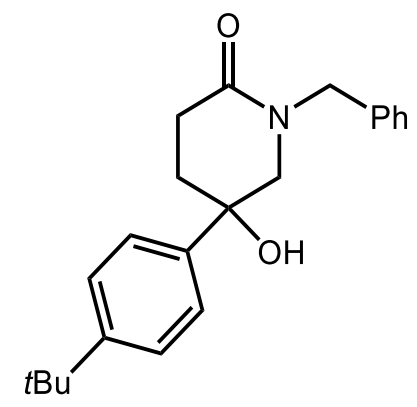

1-benzyl-5-(4-(tert-butyl)phenyl)-5-hydroxypiperidin-2-one (24): This compound was prepared according to the General Procedure using 4-(4-(tert-butyl)phenyl)pent-4-enoic acid (116 $\mathrm{mg}, 0.5 \mathrm{mmol})$, benzylamine (161 mg, $1.5 \mathrm{mmol})$ for 36 hours. After purification by column chromatography $\mathrm{SiO}_{2}(100 \%$ EtOAc to $5 \% \mathrm{MeOH} / \mathrm{EtOAc})$, the title compound was isolated as a colorless oil (132 mg, 76\% yield).

${ }^{1} \mathrm{H}$ NMR (600 MHz, $\left.\mathrm{CDCl}_{3}\right): \delta$ 7.35-7.19 (m, $\left.9 \mathrm{H}\right), 4.68(\mathrm{~d}, J=14.6 \mathrm{~Hz}, 1 \mathrm{H}), 4.40(\mathrm{~d}, J=14.6$ $\mathrm{Hz}, 1 \mathrm{H}), 3.45(\mathrm{~d}, J=12.9 \mathrm{~Hz}, 1 \mathrm{H}), 3.25(\mathrm{dd}, \mathrm{J}=12.9,2.0 \mathrm{~Hz}, 1 \mathrm{H}), 2.84-2.76(\mathrm{~m}, 1 \mathrm{H}), 2.47-$ 2.39 (m, $1 \mathrm{H}), 2.34-2.25(\mathrm{~m}, 1 \mathrm{H}), 2.07-1.98(\mathrm{~m}, 1 \mathrm{H}), 1.27$ (s, $9 \mathrm{H}) ;{ }^{13} \mathrm{C} \mathrm{NMR}\left(150 \mathrm{MHz}, \mathrm{CDCl}_{3}\right)$ : $\delta 169.6,150.4,141.5,136.6,128.5,128.0,127.3,125.2,124.5,70.3,58.6,50.2,34.3,32.8,31.2$ 28.6; IR (neat): 3392, 3060, 3032, 2962, 2934, 2866, 1615, 1495, 1213, 822, $698 \mathrm{~cm}^{-1}$; HRMS (ESI) $\mathrm{m} / \mathrm{z}$ calcd for $\mathrm{C}_{22} \mathrm{H}_{28} \mathrm{NO}_{2}\left[(\mathrm{M}+\mathrm{H})^{+}\right] 338.2120$, found 338.2123 


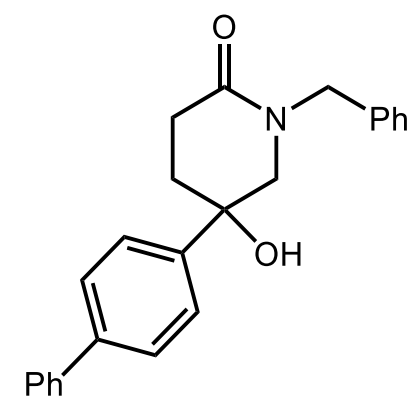

5-([1,1'-biphenyl]-4-yl)-1-benzyl-5-hydroxypiperidin-2-one (25): This compound was prepared according to the General Procedure using 4-([1,1'-biphenyl]-4-yl)pent-4-enoic acid (126 mg, 0.5 mmol), benzylamine (161 $\mathrm{mg}, 1.5 \mathrm{mmol})$ for 36 hours. After purification by column chromatography $\mathrm{SiO}_{2}(100 \%$ EtOAc to $5 \% \mathrm{MeOH} / \mathrm{EtOAc})$, the title compound was isolated as a colorless oil (102 mg, 53\% yield).

${ }^{1} \mathrm{H}$ NMR (600 MHz, $\left.\mathrm{CDCl}_{3}\right): \delta 7.55-7.49(\mathrm{~m}, 4 \mathrm{H}), 7.46(\mathrm{~d}, J=8.5 \mathrm{~Hz}, 2 \mathrm{H}), 7.42(\mathrm{t}, J=7.7 \mathrm{~Hz}$, $2 \mathrm{H}), 7.36-7.26(\mathrm{~m}, 3 \mathrm{H}), 7.26-7.21(\mathrm{~m}, 3 \mathrm{H}), 4.68(\mathrm{~d}, J=14.9 \mathrm{~Hz}, 1 \mathrm{H}), 4.47(\mathrm{~d}, J=14.9 \mathrm{~Hz}, 1$ H), $3.50(\mathrm{~d}, J=12.9 \mathrm{~Hz}, 1 \mathrm{H}), 3.35($ br s, $1 \mathrm{H}), 3.29(\mathrm{~d}, J=12.9 \mathrm{~Hz}, 1 \mathrm{H}), 2.89-2.78(\mathrm{~m}, 1 \mathrm{H})$, 2.53-2.45 (m, $1 \mathrm{H}), 2.40-2.31$ (m, $1 \mathrm{H}), 2.12-2.04(\mathrm{~m}, 1 \mathrm{H}) ;{ }^{13} \mathrm{C} \mathrm{NMR}\left(150 \mathrm{MHz}, \mathrm{CDCl}_{3}\right): \delta 169.4$, $143.4,140.6,140.3,136.6,128.8,128.6,128.1,127.4,127.1,127.0,125.3,70.6,58.6,50.2,33.0$, 28.6; IR (neat): 3254, 3058, 3031, 2955, 2924, 1609, 1485, 1236, 837, $694 \mathrm{~cm}^{-1}$; HRMS (ESI) m/z calcd for $\mathrm{C}_{24} \mathrm{H}_{24} \mathrm{NO}_{2}\left[(\mathrm{M}+\mathrm{H})^{+}\right]$358.1807, found 358.1826.<smiles>O=C1CCC(O)CN1Cc1ccccc1</smiles>

1-benzyl-5-hydroxypiperidin-2-one (26): This compound was prepared according to the General Procedure using KI (83 mg, $100 \mathrm{~mol} \%$ ), 4-pentenoic acid (50 mg, $0.5 \mathrm{mmol})$, benzylamine (161 
$\mathrm{mg}, 1.5 \mathrm{mmol})$. After purification by column chromatography $\mathrm{SiO}_{2}(100 \%$ EtOAc to $5 \%$ $\mathrm{MeOH} / \mathrm{EtOAc}$ ), the title compound was isolated as a colorless oil (53 mg, $52 \%$ yield).

${ }^{1} \mathrm{H}$ NMR (600 MHz, $\left.\mathrm{CDCl}_{3}\right): \delta$ 7.31-7.14 (m, $\left.5 \mathrm{H}\right), 4.56(\mathrm{~d}, J=14.6 \mathrm{~Hz}, 1 \mathrm{H}), 4.44(\mathrm{~d}, J=14.6$ $\mathrm{Hz}, 1 \mathrm{H}), 4.06-3.98(\mathrm{~m}, 1 \mathrm{H}), 3.29(\mathrm{dd}, J=12.5,4.2 \mathrm{~Hz}, 1 \mathrm{H}), 3.09(\mathrm{dd}, J=12.5,4.2 \mathrm{~Hz}, 1 \mathrm{H})$, 3.05 (br s, 1H), 2.66-2.55 (m, $1 \mathrm{H}), 2.39-2.31(\mathrm{~m}, 1 \mathrm{H}), 1.94-1.80(\mathrm{~m}, 2 \mathrm{H}) ;{ }^{13} \mathrm{C} \mathrm{NMR}(150 \mathrm{MHz}$, $\left.\mathrm{CDCl}_{3}\right): \delta 169.5,136.6,128.6,127.9,127.4,63.6,53.6,50.0,28.5,28.1$; IR (neat): 3274, 3027, 2953, 2932, 2898, 1609, 1497, 1274, 965, $732 \mathrm{~cm}^{-1}$; HRMS (ESI) m/z calcd for $\mathrm{C}_{12} \mathrm{H}_{16} \mathrm{NO}_{2}$ $\left[(\mathrm{M}+\mathrm{H})^{+}\right]$206.1181, found 206.1176.

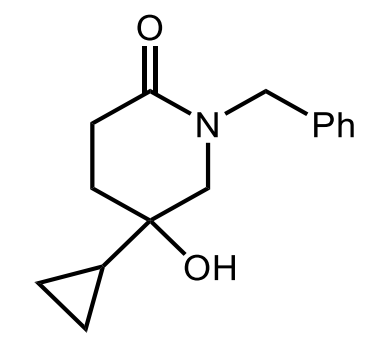

1-benzyl-5-cyclopropyl-5-hydroxypiperidin-2-one (27): This compound was prepared according to the General Procedure using 4-cyclopropylpent-4-enoic acid (70 $\mathrm{mg}, 0.5 \mathrm{mmol}$ ), benzylamine (161 mg, $1.5 \mathrm{mmol}$ ) for 36 hours. After purification by column chromatography $\mathrm{SiO}_{2}$ (100\% EtOAc to $5 \% \mathrm{MeOH} / \mathrm{EtOAc}$ ), the title compound was isolated as a colorless oil (41 mg, $34 \%$ yield).

${ }^{1} \mathrm{H}$ NMR (600 MHz, $\left.\mathrm{CDCl}_{3}\right): \delta$ 7.35-7.19 (m, $\left.5 \mathrm{H}\right), 4.61(\mathrm{~d}, J=14.9 \mathrm{~Hz}, 1 \mathrm{H}), 4.50(\mathrm{~d}, J=14.9$ $\mathrm{Hz}, 1 \mathrm{H}), 3.25(\mathrm{~d}, J=12.9 \mathrm{~Hz}, 1 \mathrm{H}), 3.04(\mathrm{~d}, J=12.9 \mathrm{~Hz}, 1 \mathrm{H}), 2.71-2.62(\mathrm{~m}, 1 \mathrm{H}), 2.51-2.42(\mathrm{~m}$, $1 \mathrm{H}), 1.81-1.71(\mathrm{~m}, 2 \mathrm{H}), 0.92-0.73(\mathrm{~m}, 1 \mathrm{H}), 0.48-0.21(\mathrm{~m}, 4 \mathrm{H}) ;{ }^{13} \mathrm{C} \mathrm{NMR}\left(150 \mathrm{MHz}, \mathrm{CDCl}_{3}\right)$ : $\delta 169.4,136.8,128.6,127.9,127.3,67.6,57.7,50.1,31.5,28.2,19.1,-0.1,-0.1$; IR (neat): 3393, 
3056, 2998, 2926, 2894, 1610, 1496, 1278, 967, $699 \mathrm{~cm}^{-1}$; HRMS (ESI) m/z calcd for $\mathrm{C}_{15} \mathrm{H}_{20} \mathrm{NO}_{2}$ $\left[(\mathrm{M}+\mathrm{H})^{+}\right] 246.1494$, found 246.1505 .<smiles>CC(C)CN1CC(C)(O)c2ccccc2C1=O</smiles>

2-butyl-4-hydroxy-4-methyl-3,4-dihydroisoquinolin-1(2H)-one (28): This compound was prepared according to the General Procedure using 2-(prop-1-en-2-yl)benzoic acid (81 mg, 0.5 mmol), butylamine (110 mg, $1.5 \mathrm{mmol})$ for 36 hours. After purification by column chromatography $\mathrm{SiO}_{2}(100 \%$ EtOAc to $5 \% \mathrm{MeOH} / \mathrm{EtOAc})$, the title compound was isolated as a colorless oil (74 mg, 63\% yield).

${ }^{1} \mathrm{H}$ NMR (600 MHz, $\left.\mathrm{CDCl}_{3}\right):$ 8 7.69-7.60 (m, $\left.1 \mathrm{H}\right)$, 7.46-7.38 (m, $\left.1 \mathrm{H}\right), 7.31-7.21(\mathrm{~m}, 2 \mathrm{H}), 3.82$ $(\mathrm{d}, J=11.9 \mathrm{~Hz}, 1 \mathrm{H}), 3.74(\mathrm{~d}, J=11.9 \mathrm{~Hz}, 1 \mathrm{H}), 3.54-3.18(\mathrm{~m}, 3 \mathrm{H}), 1.64-1.55(\mathrm{~m}, 2 \mathrm{H}), 1.54(\mathrm{~s}$ $3 \mathrm{H}), 1.40-1.31(\mathrm{~m}, 2 \mathrm{H}), 0.89(\mathrm{t}, J=7.3 \mathrm{~Hz}, 3 \mathrm{H}) ;{ }^{13} \mathrm{C} \mathrm{NMR}\left(150 \mathrm{MHz}, \mathrm{CDCl}_{3}\right): \delta$ 159.1, 147.7, 131.1, 130.6, 128.7, 123.4, 120.8, 89.3, 68.1, 46.9, 32.8, 22.6, 20.6, 13.9; IR (neat): 3408, 3002, 2943, 2925, 1736, 1689, 1282, 1127, 1067, 764, $619 \mathrm{~cm}^{-1}$; HRMS (ESI) m/z calcd for $\mathrm{C}_{14} \mathrm{H}_{20} \mathrm{NO}_{2}$ $\left[(\mathrm{M}+\mathrm{H})^{+}\right]$234.1494, found 234.1505.<smiles>C[C@H]1CC(=O)N(Cc2ccccc2)C[C@H]1O</smiles>

1-benzyl-5-hydroxy-4-methylpiperidin-2-one (29): This compound was prepared according to the General Procedure using stoichiometric KI (83 mg, $100 \mathrm{~mol} \%)$, 3-methylpent-4-enoic acid (57 $\mathrm{mg}, 0.5 \mathrm{mmol})$, benzylamine $(160 \mathrm{mg} 1.5 \mathrm{mmol})$. After purification by column 
chromatography $\mathrm{SiO}_{2}(50 \% \mathrm{EtOAc} / \mathrm{Hexane}$ to $100 \% \mathrm{EtOAc})$, the title compound was isolated as a mixture of diastereomers as white solid (48 $\mathrm{mg}, 44 \%$ yield, 9.2:1 dr from crude NMR).

${ }^{1} \mathrm{H} \mathrm{NMR}\left(600 \mathrm{MHz}, \mathrm{DCCl}_{3}\right) \delta$ 7.31-7.25 (m, $\left.2 \mathrm{H}_{\mathrm{maj}}+2 \mathrm{H}_{\mathrm{min}}\right), 7.25-7.21\left(\mathrm{~m}, 1 \mathrm{H}_{\mathrm{maj}}+1 \mathrm{H}_{\mathrm{min}}\right), 7.19$ $\left(\mathrm{d}, J=6.6 \mathrm{~Hz}, 2 \mathrm{H}_{\text {maj }}+2 \mathrm{H}_{\mathrm{min}}\right), 4.63\left(\mathrm{~d}, J=14.7 \mathrm{~Hz}, 1 \mathrm{H}_{\mathrm{maj}}\right), 4.58\left(\mathrm{~d}, J=15.0 \mathrm{~Hz}, 1 \mathrm{H}_{\min }\right), 4.50(\mathrm{~d}$, $\left.J=15.0 \mathrm{~Hz}, 1 \mathrm{H}_{\min }\right), 4.40\left(\mathrm{~d}, J=14.7 \mathrm{~Hz}, 1 \mathrm{H}_{\mathrm{maj}}\right), 3.91-3.87\left(\mathrm{~m}, 1 \mathrm{H}_{\mathrm{min}}\right), 3.63-3.57\left(\mathrm{~m}, 1 \mathrm{H}_{\text {maj }}\right)$, $3.32\left(\mathrm{dd}, J=12.6,4.8 \mathrm{~Hz}, 1 \mathrm{H}_{\text {maj }}+1 \mathrm{H}_{\mathrm{min}}\right), 3.18\left(\mathrm{dd}, J=12.9,3.0 \mathrm{~Hz} 1 \mathrm{H}_{\mathrm{min}}\right), 3.04(\mathrm{dd}, J=12.6$, $\left.7.2 \mathrm{~Hz}, 1 \mathrm{H}_{\text {maj }}\right), 2.66\left(\mathrm{dd}, J=18.0,6.0 \mathrm{~Hz}, 1 \mathrm{H}_{\text {maj }}+1 \mathrm{H}_{\text {min }}\right), 2.10\left(\mathrm{dd}, J=18.0,6.0 \mathrm{~Hz}, 1 \mathrm{H}_{\text {maj }}+1\right.$ $\left.\mathrm{H}_{\min }\right), 2.07-2.00\left(\mathrm{~m}, 1 \mathrm{H}_{\min }\right), 2.00-1.89\left(\mathrm{~m}, 1 \mathrm{H}_{\mathrm{maj}}\right), 1.01\left(\mathrm{~d}, \mathrm{~J}=6.0 \mathrm{~Hz}, 3 \mathrm{H}_{\min }\right) 0.98(\mathrm{~d}, J=6.0 \mathrm{~Hz}$ $\left.3 \mathrm{H}_{\mathrm{maj}}\right) ;{ }^{13} \mathrm{C} \mathrm{NMR}\left(150 \mathrm{MHz}, \mathrm{CDCl}_{3}\right) \delta 169.2,169.0,136.8,136.7,128.6,128.1,127.9,127.5$, 127.4, 69.8, 67.2, 53.3, 51.9, 49.8, 49.7, 37.0, 35.0, 34.4, 32.1, 17.3, 16.5; IR (neat): 3204, 2923.57, 1602, 1500, 1452, 1431, 1350, 1263, 1071, $905 \mathrm{~cm}^{-1}$; HRMS (ESI) $\mathrm{m} / z$ calcd for $\mathrm{C}_{13} \mathrm{H}_{18} \mathrm{NO}_{2}$ $\left[(\mathrm{M}+\mathrm{H})^{+}\right] 220.1338$, found 220.1339 .

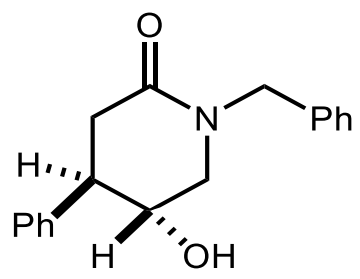

1-benzyl-5-hydroxy-4-phenylpiperidin-2-one (30): This compound was prepared according to the General Procedure using KI ( $83 \mathrm{mg}, 100 \mathrm{~mol} \%$ ), 3-phenylpent-4-enoic acid ( $88 \mathrm{mg}, 0.5 \mathrm{mmol}$ ), benzylamine (161 mg, $1.5 \mathrm{mmol})$. After purification by column chromatography $\mathrm{SiO}_{2}(100 \%$ EtOAc to $5 \% \mathrm{MeOH} / \mathrm{EtOAc}$ ), the title compound was isolated as a colorless oil (66 mg, 47\% yield, $>95: 5 \mathrm{dr}$ ).

${ }^{1} \mathrm{H}$ NMR (600 MHz, $\left.\mathrm{CDCl}_{3}\right):$ 8 7.35-7.28 (m, $\left.4 \mathrm{H}\right)$, 7.28-7.22 (m, $\left.5 \mathrm{H}\right)$, 7.20-7.16 (m, $\left.2 \mathrm{H}\right), 4.66$ $(\mathrm{d}, J=14.6 \mathrm{~Hz}, 1 \mathrm{H}), 4.50(\mathrm{~d}, J=14.6 \mathrm{~Hz}, 1 \mathrm{H}), 4.07-4.00(\mathrm{~m}, 1 \mathrm{H}), 3.37(\mathrm{dd}, J=12.5,5.1 \mathrm{~Hz}, 1$ 
H), $3.13(\mathrm{dd}, J=12.5,8.1 \mathrm{~Hz}, 1 \mathrm{H}), 3.09-3.02(\mathrm{~m}, 1 \mathrm{H}), 2.83(\mathrm{dd}, J=17.8,5.6 \mathrm{~Hz}, 1 \mathrm{H}), 2.63(\mathrm{dd}$, $J=17.8,10.3 \mathrm{~Hz}, 1 \mathrm{H}), 2.16(\mathrm{br} \mathrm{s}, 1 \mathrm{H}) ;{ }^{13} \mathrm{C} \mathrm{NMR}\left(150 \mathrm{MHz}, \mathrm{CDCl}_{3}\right): \delta 168.7,139.5,136.6,129.1$, 128.7, 128.3, 127.6, 127.6, 127.5, 69.1, 51.7, 50.0, 46.5, 36.4; IR (neat): 3288, 2972, 2882, 1607 , $1451,1338,1089,1045,879 \mathrm{~cm}^{-1}$; HRMS (ESI) $\mathrm{m} / z$ calcd for $\mathrm{C}_{18} \mathrm{H}_{20} \mathrm{NO}_{2}\left[(\mathrm{M}+\mathrm{H})^{+}\right] 282.1494$, found 282.1480 .

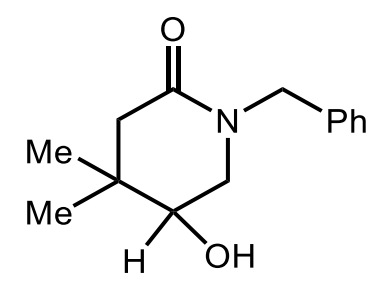

1-benzyl-5-hydroxy-4,4-dimethylpiperidin-2-one (31): This compound was prepared according to the General Procedure using stoichiometric KI (83 mg, $100 \mathrm{~mol} \%)$, 3,3-dimethylpent-4-enoic acid $(57 \mathrm{mg}, 0.5 \mathrm{mmol})$, benzylamine $(160 \mathrm{mg} 1.5 \mathrm{mmol})$. After purification by column chromatography $\mathrm{SiO}_{2}(50 \% \mathrm{EtOAc} / \mathrm{Hexane}$ to $100 \% \mathrm{EtOAc})$, the title compound was isolated as a colorless oil (56 mg, $48 \%$ yield).

${ }^{1} \mathrm{H}$ NMR $\left(600 \mathrm{MHz}, \mathrm{DCCl}_{3}\right) \delta$ 7.28-7.17 (m, $\left.5 \mathrm{H}\right), 4.57(\mathrm{~d}, J=18.0 \mathrm{~Hz}, 1 \mathrm{H}), 4.46(\mathrm{~d}, J=18.0 \mathrm{~Hz}$, $1 \mathrm{H}), 3.58(\mathrm{br} \mathrm{s}, 1 \mathrm{H}), 3.34-3.28(\mathrm{~m}, 1 \mathrm{H}), 3.05(\mathrm{dd}, J=13.2,5.4 \mathrm{~Hz}, 1 \mathrm{H}), 2.45(\mathrm{~d}, J=16.8 \mathrm{~Hz}, 1$ H), 2.14 (d, $J=17.4 \mathrm{~Hz}, 1 \mathrm{H}), 1.95$ (br s, 1H), 0.93 (s, $3 \mathrm{H}), 0.92$ (s, 3H); ${ }^{13} \mathrm{C}$ NMR (150 MHz, $\left.\mathrm{CDCl}_{3}\right) \delta 169.1,136.8,128.6,128.0,127.4,71.3,50.7,49.7,42.3,34.6,25.7,22.3$; IR (neat): $3315,2870,1613,1496,1452,1357,1260,1083,908 \mathrm{~cm}^{-1}$; HRMS (ESI) $\mathrm{m} / z$ calcd for $\mathrm{C}_{14} \mathrm{H}_{20} \mathrm{NO}_{2}$ $\left[(\mathrm{M}+\mathrm{H})^{+}\right]$234.1494, found 234.1502. 


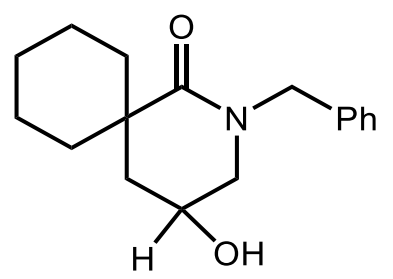

2-benzyl-4-hydroxy-2-azaspiro[5.5] undecan-1-one (32): This compound was prepared according to the General Procedure using stoichiometric KI (83 mg, $100 \mathrm{~mol} \%$, 1allylcyclohexane-1-carboxylic acid ( $84 \mathrm{mg}, 0.5 \mathrm{mmol})$, benzylamine (160 mg $1.5 \mathrm{mmol})$ to afford desired amino lactone compound. Isolated amino lactone compound was treated with $\mathrm{AlMe}_{3}(0.5$ $\mathrm{mL}, 1 \mathrm{M}$ solution in THF, $0.5 \mathrm{mmol}$ ) and stirred at $60{ }^{\circ} \mathrm{C}$ for $16 \mathrm{~h}$. The reaction was cooled to the room temperature, diluted with $2 \mathrm{~mL}$ of EtOAc and quenched with $2 \mathrm{~mL}$ of water. Organic layer was separated, and the aqueous layer was extracted with EtOAc $(2 \times 2 \mathrm{~mL})$. Combined organic layer was dried over anhydrous $\mathrm{Na}_{2} \mathrm{SO}_{4}$ and evaporated under reduced pressure. After purification by column chromatography $\mathrm{SiO}_{2}(50 \%$ EtOAc/Hexane to $100 \%$ EtOAc), the title compound was isolated as a colorless oil ( $85 \mathrm{mg}, 62 \%$ yield).

${ }^{1} \mathrm{H}$ NMR $\left(600 \mathrm{MHz}, \mathrm{DCCl}_{3}\right) \delta$ 7.30-7.25 (m, $\left.2 \mathrm{H}\right), 7.25-7.21(\mathrm{~m}, 1 \mathrm{H}), 7.16(\mathrm{~d}, J=6.6 \mathrm{~Hz}, 2 \mathrm{H})$, $4.66(\mathrm{~d}, J=14.6 \mathrm{~Hz}, 1 \mathrm{H}), 4.37(\mathrm{~d}, J=14.6 \mathrm{~Hz}, 1 \mathrm{H}), 4.07-3.98(\mathrm{~m}, 1 \mathrm{H}), 3.34-3.28(\mathrm{~m}, 1 \mathrm{H}), 3.02$ $(\mathrm{dd}, J=12.0,9.0 \mathrm{~Hz}, 1 \mathrm{H}), 2.34-2.27(\mathrm{~m}, 1 \mathrm{H}), 2.12-2.03(\mathrm{~m}, 2 \mathrm{H}), 1.84-1.75(\mathrm{~m}, 1 \mathrm{H}), 1.71-1.50$ $(\mathrm{m}, 5 \mathrm{H}), 1.50-1.40(\mathrm{~m}, 2 \mathrm{H}), 1.35-1.26(\mathrm{~m}, 2 \mathrm{H}) ;{ }^{13} \mathrm{C} \mathrm{NMR}\left(150 \mathrm{MHz}, \mathrm{CDCl}_{3}\right) \delta 175.5,137.2$, 128.6, 127.9, 127.4, 62.7, 53.4, 50.6, 41.8, 38.2, 36.1, 33.7, 25.5, 21.0; IR (neat): 3409.78, 2922.36, 2858.31, 1606.76, 1494.84, 1452.13, 1365.39, 1168.53, 1501.12, $737.30 \mathrm{~cm}^{-1}$; HRMS (ESI) $\mathrm{m} / z$ calcd for $\mathrm{C}_{17} \mathrm{H}_{24} \mathrm{NO}_{2}\left[(\mathrm{M}+\mathrm{H})^{+}\right]$274.1807, found 274.1796. 


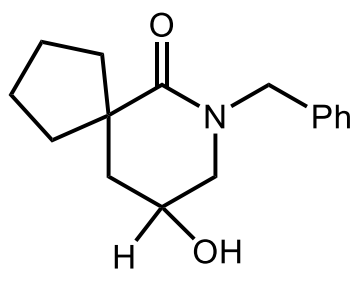

7-benzyl-9-hydroxy-7-azaspiro[4.5]decan-6-one (33): This compound was prepared according to the General Procedure using stoichiometric KI (83 mg, $100 \mathrm{~mol} \%)$, 1-allylcyclopentane-1carboxylic acid (77 $\mathrm{mg}, 0.5 \mathrm{mmol})$, benzylamine (160 $\mathrm{mg} 1.5 \mathrm{mmol})$ to afford desired amino lactone compound. Isolated amino lactone compound was treated with $\mathrm{AlMe}_{3}(0.5 \mathrm{~mL}, 1 \mathrm{M}$ solution in THF, $0.5 \mathrm{mmol}$ ) and stirred at $60{ }^{\circ} \mathrm{C}$ for $16 \mathrm{~h}$. The reaction was cooled to the room temperature, diluted with $2 \mathrm{~mL}$ of EtOAc and quenched with $2 \mathrm{~mL}$ of water. Organic layer was separated, and the aqueous layer was extracted with EtOAc $(2 \times 2 \mathrm{~mL})$. Combined organic layer was dried over anhydrous $\mathrm{Na}_{2} \mathrm{SO}_{4}$ and evaporated under reduced pressure. After purification by column chromatography $\mathrm{SiO}_{2}(50 \%$ EtOAc/Hexane to $100 \%$ EtOAc), the title compound was isolated as a colorless oil (75 $\mathrm{mg}, 58 \%$ yield).

${ }^{1} \mathrm{H}$ NMR $\left(600 \mathrm{MHz}, \mathrm{DCCl}_{3}\right) \delta$ 7.31-7.25 (m, $\left.2 \mathrm{H}\right), 7.25-7.20(\mathrm{~m}, 1 \mathrm{H}), 7.16(\mathrm{~d}, J=9.0 \mathrm{~Hz}, 2 \mathrm{H})$, $4.65(\mathrm{~d}, J=15.0 \mathrm{~Hz}, 1 \mathrm{H}), 4.32(\mathrm{~d}, J=15.0 \mathrm{~Hz}, 1 \mathrm{H}), 4.04-3.95(\mathrm{~m}, 1 \mathrm{H}), 3.37-3.28(\mathrm{~m}, 1 \mathrm{H}), 3.05$ $(\mathrm{dd}, J=12.0,9.0 \mathrm{~Hz}, 1 \mathrm{H}), 2.35-2.26(\mathrm{~m}, 1 \mathrm{H}), 1.97-1.88(\mathrm{~m}, 2 \mathrm{H}), 1.86-1.75(\mathrm{~m}, 2 \mathrm{H}), 1.75-1.68$ (m, $1 \mathrm{H}), 1.66-1.53(\mathrm{~m}, 3 \mathrm{H}), 1.48-1.38(\mathrm{~m}, 1 \mathrm{H}) ;{ }^{13} \mathrm{C} \mathrm{NMR}\left(150 \mathrm{MHz}, \mathrm{CDCl}_{3}\right) \delta$ 176.0, 137.1, 128.6, 127.8, 127.4, 63.2, 53.7, 50.5, 48.1, 43.7, 40.2, 39.5, 25.9, 25.7; IR (neat): 3390.53, 2928.11, 2859.40, 1610.43, 1494.88, 1426.41, 1235.52, 1057.83, $738.03 \mathrm{~cm}^{-1}$; HRMS (ESI) $\mathrm{m} / z$ calcd for $\mathrm{C}_{16} \mathrm{H}_{22} \mathrm{NO}_{2}\left[(\mathrm{M}+\mathrm{H})^{+}\right] 260.1651$, found 260.1639 . 


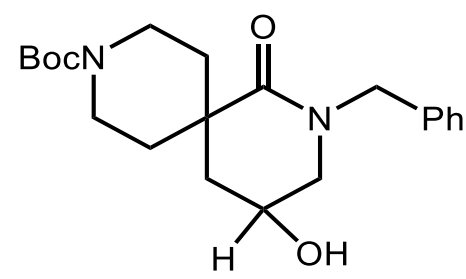

tert-butyl 2-benzyl-4-hydroxy-1-oxo-2,9-diazaspiro[5.5] undecane-9-carboxylate (34): This compound was prepared according to the General Procedure using stoichiometric KI (83 mg, 100 mol \%), 4-allyl-1-(tert-butoxycarbonyl)piperidine-4-carboxylic acid (135 mg, $0.5 \mathrm{mmol}$ ), benzylamine (160 mg $1.5 \mathrm{mmol}$ ) to afford desired amino lactone compound. Isolated amino lactone compound was treated with $\mathrm{AlMe}_{3}(0.5 \mathrm{~mL}, 1 \mathrm{M}$ solution in THF, $0.5 \mathrm{mmol})$ and stirred at $60{ }^{\circ} \mathrm{C}$ for $16 \mathrm{~h}$. The reaction was cooled to the room temperature, diluted with $2 \mathrm{~mL}$ of EtOAc and quenched with $2 \mathrm{~mL}$ of water. Organic layer was separated, and the aqueous layer was extracted with EtOAc $(2 \times 2 \mathrm{~mL})$. Combined organic layer was dried over anhydrous $\mathrm{Na}_{2} \mathrm{SO}_{4}$ and evaporated under reduced pressure. After purification by column chromatography $\mathrm{SiO}_{2}(50 \% \mathrm{EtOAc} / \mathrm{Hexane}$ to $100 \%$ EtOAc), the title compound was isolated as a colorless oil (101 $\mathrm{mg}, 54 \%$ yield).

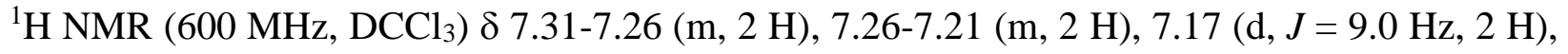
$4.64(\mathrm{~d}, J=12.0 \mathrm{~Hz}, 1 \mathrm{H}), 4.41(\mathrm{~d}, J=12.0 \mathrm{~Hz}, 1 \mathrm{H}), 4.13-4.03(\mathrm{~m}, 1 \mathrm{H}), 4.00-3.86(\mathrm{~m}, 1 \mathrm{H}), 3.86-$ $3.73(\mathrm{~m}, 1 \mathrm{H}), 3.35(\mathrm{dd}, J=12.0,3.0 \mathrm{~Hz}, 1 \mathrm{H}), 3.07(\mathrm{dd}, J=12.0,9.0 \mathrm{~Hz}, 2 \mathrm{H}), 2.24-2.17(\mathrm{~m}, 2$ $\mathrm{H}), 2.03-1.94(\mathrm{~m}, 1 \mathrm{H}), 1.77-1.61(\mathrm{~m}, 2 \mathrm{H}), 1.55-1.45(\mathrm{~m}, 2 \mathrm{H}), 1.43(\mathrm{~s}, 9 \mathrm{H}) ;{ }^{13} \mathrm{C} \mathrm{NMR}(150 \mathrm{MHz}$, $\left.\mathrm{CDCl}_{3}\right) \delta 173.9,154.8,136.9,128.7,127.9,127.5,79.6,62.3,53.4,50.6,39.6,39.0,35.3,33.9$ 29.7, 28.4; IR (neat): 3398.72, 2924.94, 2862.57, 1666.79, 1685.25, 1419.97, 1364.03, 1244.13, 1152.92, 1055.23,731.27 $\mathrm{cm}^{-1}$; HRMS (ESI) $\mathrm{m} / z$ calcd for $\mathrm{C}_{21} \mathrm{H}_{31} \mathrm{~N}_{2} \mathrm{O}_{4}\left[(\mathrm{M}+\mathrm{H})^{+}\right] 375.2284$, found 375.2289. 


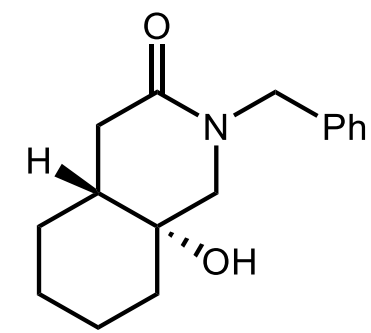

2-benzyl-8a-hydroxyoctahydroisoquinolin-3(2H)-one (35): This compound was prepared according to the General Procedure using 2-(2-methylenecyclohexyl)acetic acid (77 mg, 0.5 mmol), benzylamine (161 mg, $1.5 \mathrm{mmol})$. After purification by column chromatography $\mathrm{SiO}_{2}(100 \%$ EtOAc to $5 \% \mathrm{MeOH} / \mathrm{EtOAc}$ ), the title compound was isolated as a colorless oil ( $85 \mathrm{mg}, 60 \%$ yield, $>95: 5 \mathrm{dr})$.

${ }^{1} \mathrm{H}$ NMR $\left(600 \mathrm{MHz}, \mathrm{CDCl}_{3}\right): \delta$ 7.29-7.24 (m, $\left.2 \mathrm{H}\right), 7.22-7.17(\mathrm{~m}, 3 \mathrm{H}), 4.56(\mathrm{~d}, J=14.9 \mathrm{~Hz}, 1 \mathrm{H})$, $4.49(\mathrm{~d}, J=14.9 \mathrm{~Hz}, 1 \mathrm{H}), 3.08(\mathrm{~d}, J=12.8 \mathrm{~Hz}, 1 \mathrm{H}), 2.98(\mathrm{~d}, J=12.8 \mathrm{~Hz}, 1 \mathrm{H}), 2.35-2.25(\mathrm{~m}, 3$ H), 1.73-1.64 (m, $2 \mathrm{H}), 1.63-1.49(\mathrm{~m}, 3 \mathrm{H}), 1.48-1.41(\mathrm{~m}, 1 \mathrm{H}), 1.31-1.13(\mathrm{~m}, 3 \mathrm{H}) ;{ }^{13} \mathrm{C}$ NMR $(150$ $\left.\mathrm{MHz}, \mathrm{CDCl}_{3}\right): \delta 169.7,136.8,128.5,127.8,127.2,67.4,59.4,49.7,38.3,35.5,34.9,27.1,24.9$, 20.5; IR (neat): 3292, 3025, 2925, 2856, 1612, 1493, 1276, 957, $734 \mathrm{~cm}^{-1} ;$ HRMS (ESI) $\mathrm{m} / z$ calcd for $\mathrm{C}_{16} \mathrm{H}_{22} \mathrm{NO}_{2}\left[(\mathrm{M}+\mathrm{H})^{+}\right]$260.1651, found 260.1656.

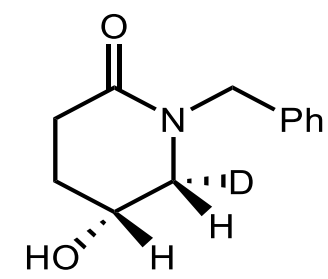

1-benzyl-5-hydroxypiperidin-2-one (36): This compound was prepared according to the General Procedure using KI (42 mg, $100 \mathrm{~mol} \%$ ), (E)-pent-4-enoic-5-d acid ${ }^{6}(25 \mathrm{mg}, 0.5 \mathrm{mmol}$ ), benzylamine $(81 \mathrm{mg}, 1.5 \mathrm{mmol})$. After purification by column chromatography $\mathrm{SiO}_{2}(100 \%$ EtOAc to $5 \% \mathrm{MeOH} / \mathrm{EtOAc}$ ), the title compound was isolated as a colorless oil (15 mg, 29\% yield). 
${ }^{1} \mathrm{H}$ NMR $\left(600 \mathrm{MHz}, \mathrm{CDCl}_{3}\right): \delta$ 7.36-7.31 (m, $\left.2 \mathrm{H}\right), 7.31-7.23(\mathrm{~m}, 3 \mathrm{H}), 4.62(\mathrm{~d}, J=14.7 \mathrm{~Hz}, 1 \mathrm{H})$, $4.55(\mathrm{~d}, J=14.7 \mathrm{~Hz}, 1 \mathrm{H}), 4.15-4.08(\mathrm{~m}, 1 \mathrm{H}), 3.15(\mathrm{~d}, J=4.8 \mathrm{~Hz}, 1 \mathrm{H}), 2.72-2.66(\mathrm{~m}, 1 \mathrm{H}), 2.49-$ 2.41 (m, $1 \mathrm{H}), 2.36$ (br s, 1H), 2.03-1.88 (m, $\left.2 \mathrm{H}) ;{ }^{13} \mathrm{C} \mathrm{NMR} \mathrm{(150} \mathrm{MHz,} \mathrm{CDCl}_{3}\right): \delta 169.4,136.7$, 128.6, 128.0, 127.4, 63.9, 53.2 (t, $J_{\mathrm{C}, \mathrm{D}}=20.9 \mathrm{~Hz}$ ), 50.0, 28.7, 28.1; IR (neat): 3274, 3027, 2953, 2932, 2898, 1609, 1497, 1274, 965, $732 \mathrm{~cm}^{-1}$; HRMS (ESI) $\mathrm{m} / \mathrm{z}$ calcd for $\mathrm{C}_{12} \mathrm{H}_{15} \mathrm{DNO}_{2}\left[(\mathrm{M}+\mathrm{H})^{+}\right]$ 207.1244, found 207.1238.

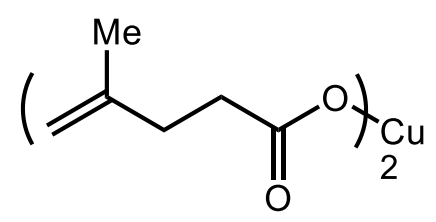

bis((4-methylpent-4-enoyl)oxy)copper (38): To a $8 \mathrm{~mL}$ vial was added $\mathrm{Cu}(\mathrm{OH})_{2}(98 \mathrm{mg}, 1.0$ mmol) and 4-methylpent-4-enoic acid (228 mg, $2.0 \mathrm{mmol})$, followed by $\mathrm{MeOH}(4 \mathrm{~mL})$. The reaction mixture was then heated to $60^{\circ} \mathrm{C}$ for 6 hours. After cooled to room temperature, $\mathrm{MeOH}$ was removed under reduced pressure and the resulting dark green solid was triturated with EtOAc. The desired product was obtained as dark green solid after filtration (226 mg, 78\% yield).

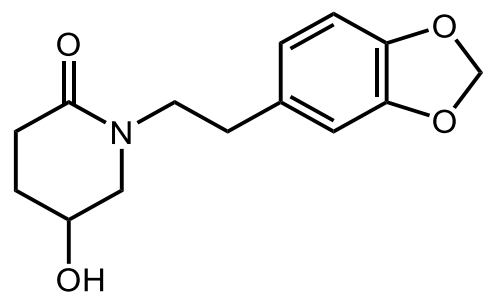

1-(2-(benzo[d][1,3]dioxol-5-yl)ethyl)-5-hydroxypiperidin-2-one (44a): This compound was prepared according to the General Procedure A using stoichiometric KI ( $83 \mathrm{mg}, 100 \mathrm{~mol} \%$ pent4-enoic acid (50 mg, $0.5 \mathrm{mmol})$, 2-(1,3-Benzodioxol-5-yl)ethanamine (248 mg $1.5 \mathrm{mmol})$. Reaction time was $48 \mathrm{~h}$. After purification by column chromatography $\mathrm{SiO}_{2}(50 \% \mathrm{EtOAc} / \mathrm{Hexane}$ to $5 \% \mathrm{MeOH} / \mathrm{EtOAc}$ ), the title compound was isolated as a white solid (69 $\mathrm{mg}, 52 \%$ yield). 
${ }^{1} \mathrm{H} \mathrm{NMR}\left(600 \mathrm{MHz}, \mathrm{DCCl}_{3}\right) \delta$ 6.77-6.71 (m, $\left.2 \mathrm{H}\right), 6.69-6.65(\mathrm{~m}, 1 \mathrm{H}), 5.94(\mathrm{~s}, 2 \mathrm{H}), 4.11-4.06(\mathrm{~m}$, $1 \mathrm{H}), 3.58-3.45(\mathrm{~m}, 2 \mathrm{H}), 3.32(\mathrm{dd}, J=12.0,3.0 \mathrm{~Hz}, 1 \mathrm{H}), 3.10(\mathrm{dd}, J=12.0,3.0 \mathrm{~Hz}, 1 \mathrm{H}), 2.80(\mathrm{t}$, $J=9.0 \mathrm{~Hz}, 2 \mathrm{H}), 2.64-2.56(\mathrm{~m}, 1 \mathrm{H}), 2.43-2.34(\mathrm{~m}, 1 \mathrm{H}), 2.10(\mathrm{br} \mathrm{s}, 1 \mathrm{H}), 2.00-1.92(\mathrm{~m}, 1 \mathrm{H}), 1.90-$ $1.84(\mathrm{~m}, 1 \mathrm{H}) ;{ }^{13} \mathrm{C}$ NMR $\left(150 \mathrm{MHz}, \mathrm{CDCl}_{3}\right) \delta 168.9,147.7,146.1,132.8,121.7,109.2,108.3$, 100.9, 64.2, 55.1, 49.4, 33.2, 28.8, 28.1; IR (neat): 3293, 3062, 3021, 2930, 1612, 1503, 1314, 1220, 1125, 1031, $734 \mathrm{~cm}^{-1}$; HRMS (ESI) $\mathrm{m} / z$ calcd for $\mathrm{C}_{14} \mathrm{H}_{18} \mathrm{NO}_{4}\left[(\mathrm{M}+\mathrm{H})^{+}\right]$264.1236, found 264.1219.

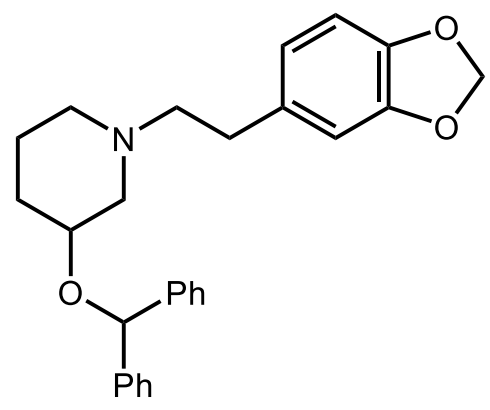

3-(benzhydryloxy)-1-(2-(benzo[d][1,3]dioxol-5-yl)ethyl)piperidine (44): To a $8 \mathrm{ml}$ vial equipped with magnetic stir bar was added $37(53 \mathrm{mg}, 0.2 \mathrm{mmol})$ and anhydrous THF (1 mL). Then $\mathrm{AlCl}_{3}(40 \mathrm{mg}, 0.3 \mathrm{mmol})$ was added, followed by $\mathrm{LiAlH}_{4}(12 \mathrm{mg}, 0.3 \mathrm{mmol})$. The reaction mixture was stirred at room temperature for $16 \mathrm{~h}$. Then the reaction was quenched with aqueous $\mathrm{NaOH}$ solution $(0.5 \mathrm{~mL}, 1 \mathrm{M})$, and the organic layer was separated. The aqueous layer was extracted with EtOAc $(2 \times 2 \mathrm{~mL})$, and the combined organic layers was dried over anhydrous $\mathrm{Na}_{2} \mathrm{SO}_{4}$, concentrated under reduced pressure to afford crude product as a yellow oil, which was directly used in next step without further purification. Then to a $8 \mathrm{~mL}$ vial with crude products was added benzhydryl chloride $(0.4 \mathrm{~mL})$, followed by $\mathrm{Ag}_{2} \mathrm{CO}_{3}(110 \mathrm{mg}, 0.4 \mathrm{mmol})$. The reaction mixture was heated to $100{ }^{\circ} \mathrm{C}$ for 2 hours. After cooling to room temperature, the reaction mixture was diluted with EtOAc $(5 \mathrm{~mL})$, and $2 \mathrm{~mL}$ water was added to the mixture. The organic layer was 
separated, and the aqueous layer was extracted with EtOAc $(2 \mathrm{~mL} \times 2)$. The combined organic layer was dried over $\mathrm{Na}_{2} \mathrm{SO}_{4}$, concentrated under reduced pressure to afford crude product as brown oil. The crude product was purified by column chromatography $\mathrm{SiO}_{2}(20 \%$ EtOAc in Hexane to $50 \%$ EtOAc in hexane) to afford 38 as yellow oil. (48 $\mathrm{mg}, 58 \%$ yield). Spectral data for this compound was previously reported and matched with the current data. ${ }^{7}$

${ }^{1} \mathrm{H}$ NMR $\left(600 \mathrm{MHz}, \mathrm{DCCl}_{3}\right) \delta$ 7.39-7.32 (m, $\left.8 \mathrm{H}\right), 7.29-7.24(\mathrm{~m}, 2 \mathrm{H}), 6.75(\mathrm{~d}, J=7.8 \mathrm{~Hz}, 1 \mathrm{H})$, $6.70(\mathrm{~d}, J=1.8 \mathrm{~Hz}, 1 \mathrm{H}), 6.65(\mathrm{dd}, J=7.8,1.8 \mathrm{~Hz}, 1 \mathrm{H}), 5.94(\mathrm{~s}, 2 \mathrm{H}), 5.58(\mathrm{~s}, 1 \mathrm{H}), 3.63-3.54(\mathrm{~m}$, $1 \mathrm{H}), 3.16-3.07$ (m, $1 \mathrm{H}), 2.87-2.76(\mathrm{~m}, 1 \mathrm{H}), 2.75-2.68$ (m, $2 \mathrm{H}), 2.64-2.54(\mathrm{~m}, 2 \mathrm{H}), 2.15-2.01$ (m, $3 \mathrm{H}), 1.81-1.73(\mathrm{~m}, 1 \mathrm{H}), 1.56-1.45(\mathrm{~m}, 1 \mathrm{H}), 1.42-1.32(\mathrm{~m}, 1 \mathrm{H}) ;{ }^{13} \mathrm{C} \mathrm{NMR}\left(150 \mathrm{MHz}, \mathrm{CDCl}_{3}\right)$ $\delta 147.5,145.7,142.7,142.6,134.2,128.4,128.3,127.4,127.3,127.1,127.1,121.4,109.1,108.2$, $100.8,81.0,77.2,76.8,73.0,60.8,58.6,53.3,33.2,30.7,23.5 ;$ IR (neat): 3063, 3028, 2937, $2800,1735,1488,1441,1242,1039,697 \mathrm{~cm}^{-1}$ 


\section{References:}

1. Nicolai, S.; Piemontesi, C.; Waser, J. A Palladium-Catalyzed Aminoalkynylation Strategy towards Bicyclic Heterocycles: Synthesis of ( \pm )-Trachelanthamidine. Angew. Chem. Int. Ed. 2011, $50,4680-4683$.

2. Gao, Y.; Li, X.; Chen, W.; Tang, G.; Zhao, Y. Copper-Catalyzed Phosphonation-Annulation Approaches to the Synthesis of $\beta$-Phosphonotetrahydrofurans Involving $\mathrm{C}-\mathrm{P}$ and $\mathrm{C}-\mathrm{O}$ Bonds Formation. J. Org. Chem. 2015, 80, 11398-11406.

3. Nishikawa, Y.; Kimura, S.; Kato, Y.; Yamazaki, N.; Hara, O. Pd(II)-Catalyzed Allylic C-H Amination for the Preparation of 1,2- and 1,3-Cyclic Ureas. Org. Lett. 2015, 17, 888-891

4. Ferreira, J. A.; Solano, D.M.; Oakdale, J. S.; Kurth, M. J. Novel Trinitrogen-Containing Triheterocycles via the Intramolecular Nitrile Oxide Cycloaddition Reaction. Synthesis 2011, 20, $3241-3246$.

5. Hemric, B. N.; Shen, K.; Wang, Q. Copper-Catalyzed Amino Lactonization and Amino Oxygenation of Alkenes Using O-Benzoylhydroxylamines. J. Am. Chem. Soc. 2016, 138, 58135816.

6. Conway, J. H.; and Rovis, T. Regiodivergent Iridium(III)-Catalyzed Diamination ofAlkenyl Amides with Secondary Amines: Complementary Access to $\gamma$ - or $\delta$-Lactams. J. Am. Chem. Soc. 2018, $140,135-138$.

7. Cossy, J.; Dumas, C.; Gomez Pardo, D. A Short and Efficient Synthesis of Zamifenacin a Muscarinic M3 Receptor Antagonist. Biorg. Med. Chem. Lett. 1997, 7, 1343-1344. 


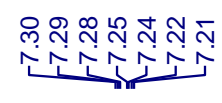<smiles>CC1(O)CCC(=O)N(Cc2ccccc2)C1</smiles>
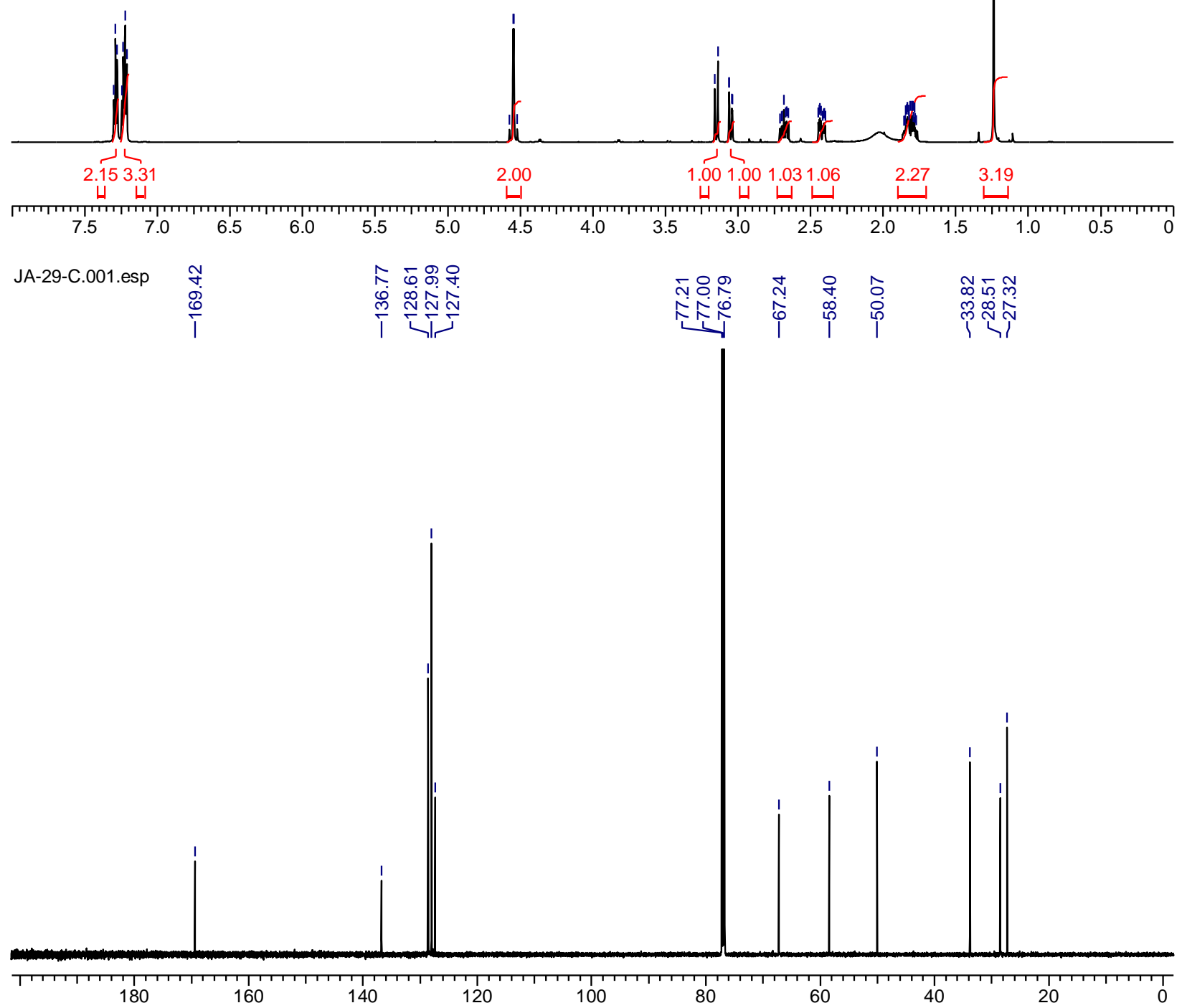


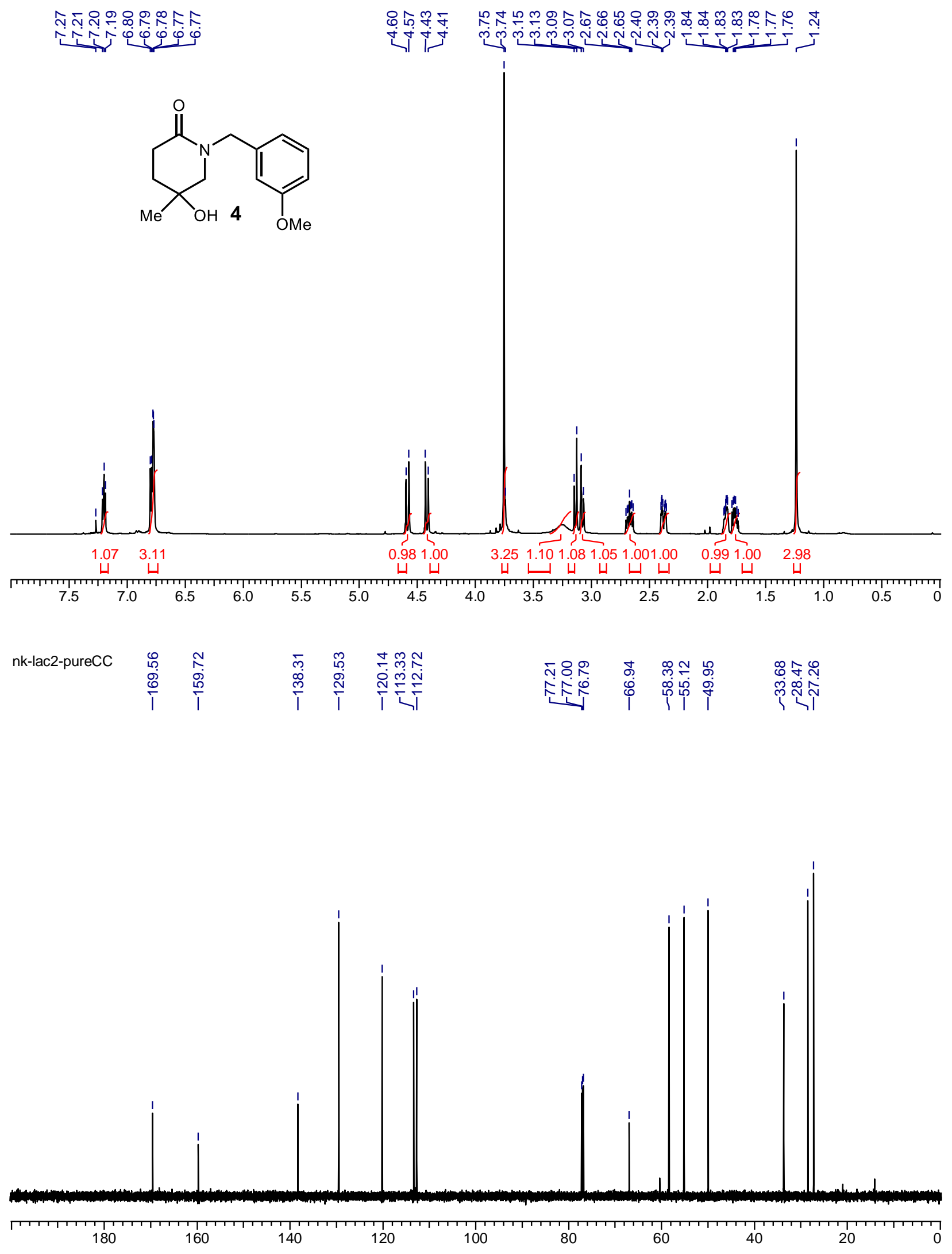


ำรำำำำ

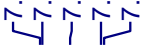

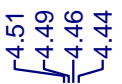

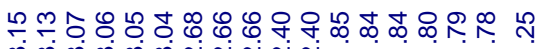

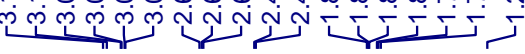<smiles>CC1(O)CCC(=O)N(Cc2ccc(Br)cc2)C1</smiles>

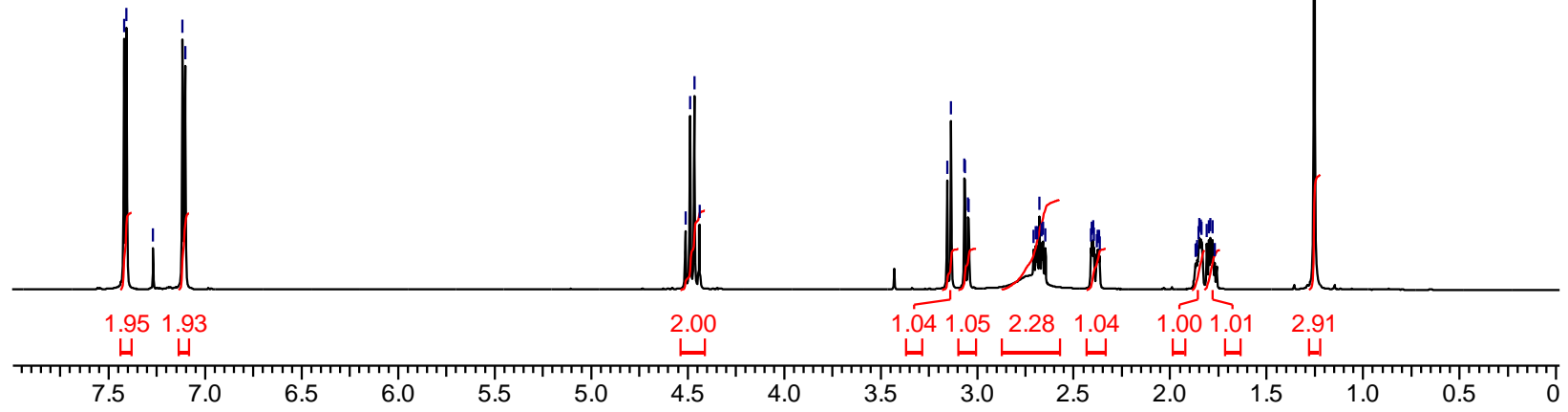

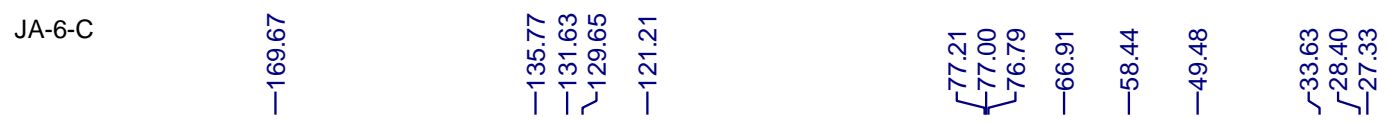

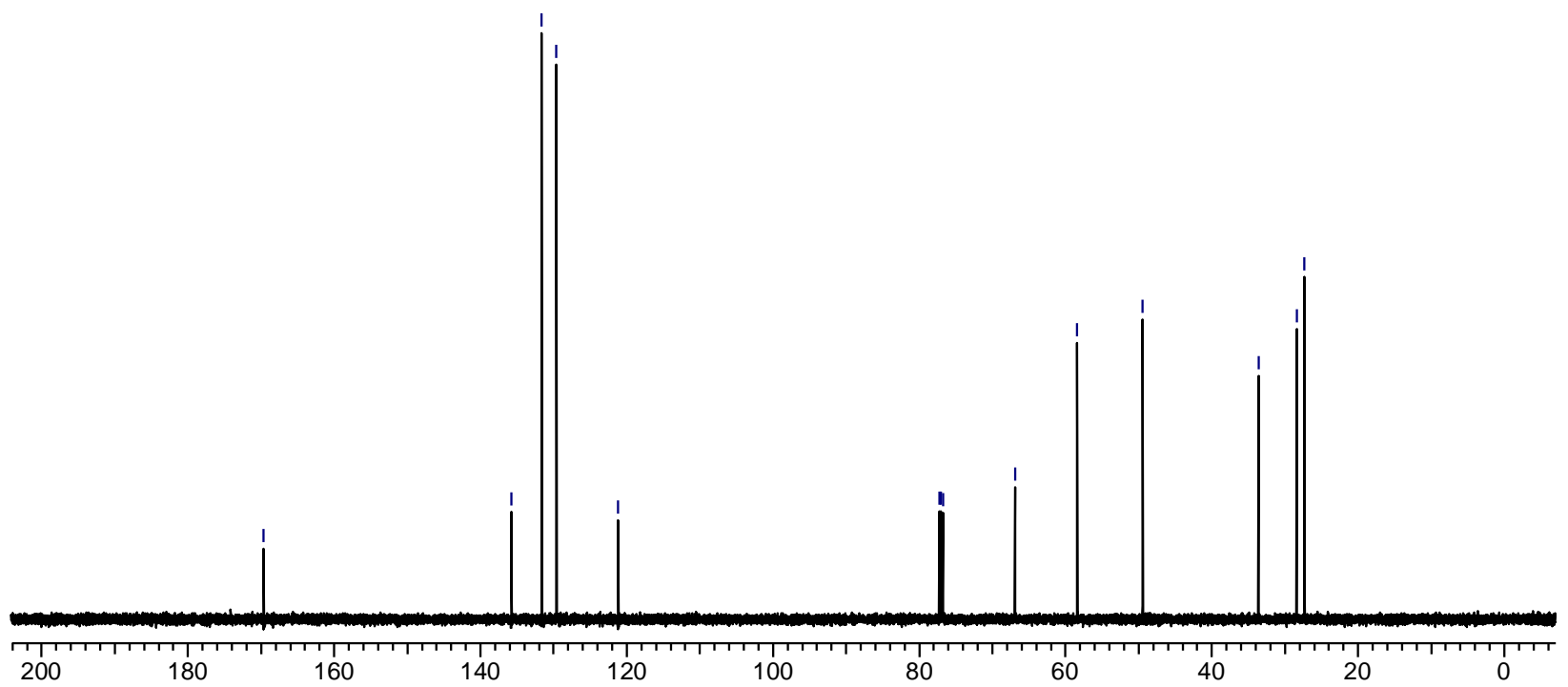




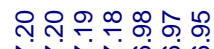

$$
\begin{aligned}
& \text { 近尔 }
\end{aligned}
$$

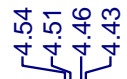

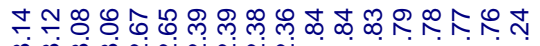<smiles>CC1(O)CCC(=O)N(Cc2ccc(F)cc2)C1</smiles>
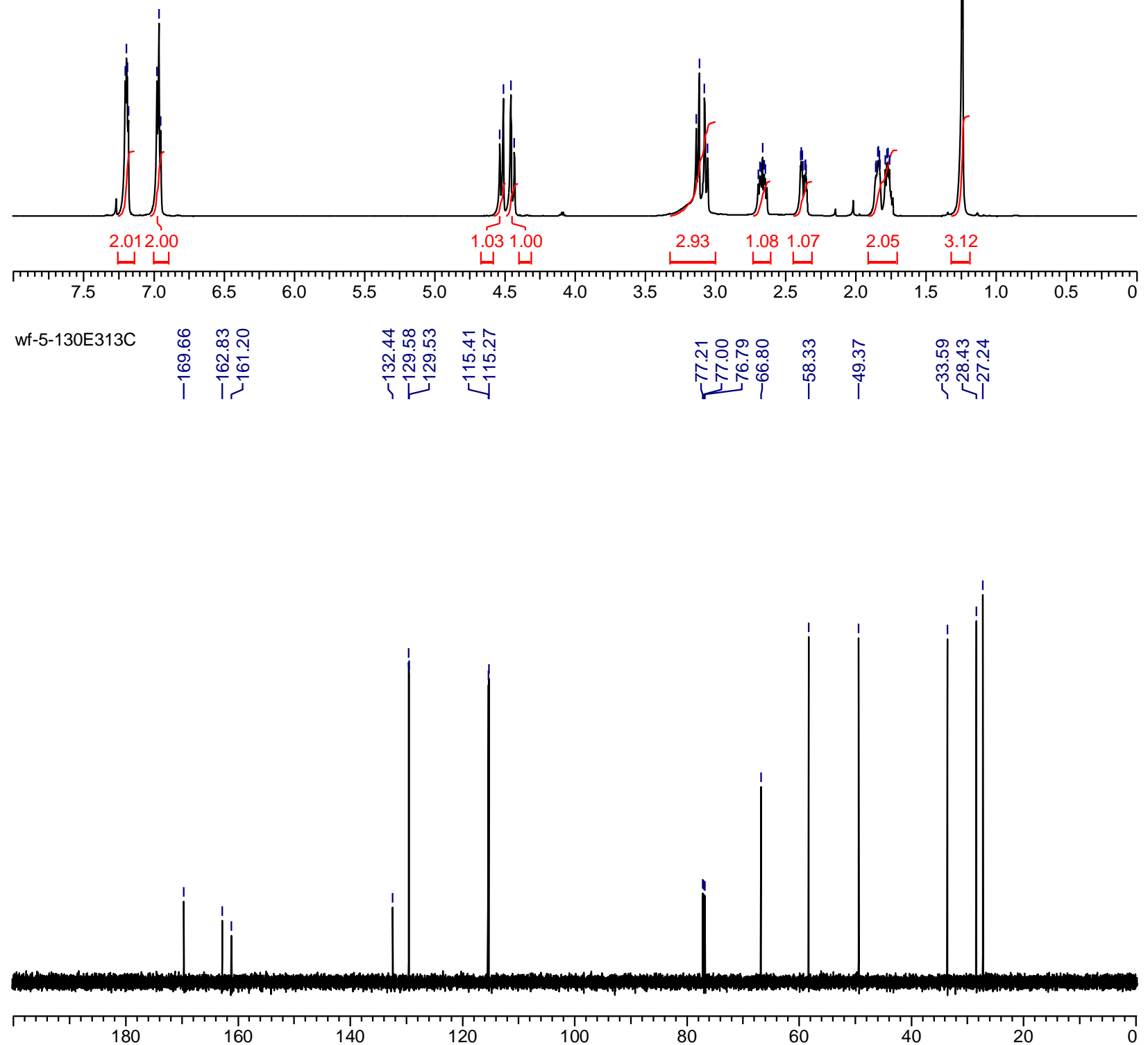


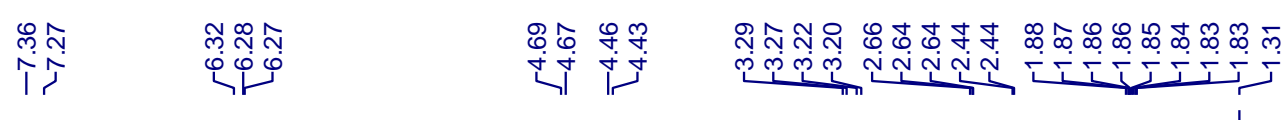<smiles>[3H]C1(O)CCC(=O)N(Cc2ccoc2)C1</smiles>

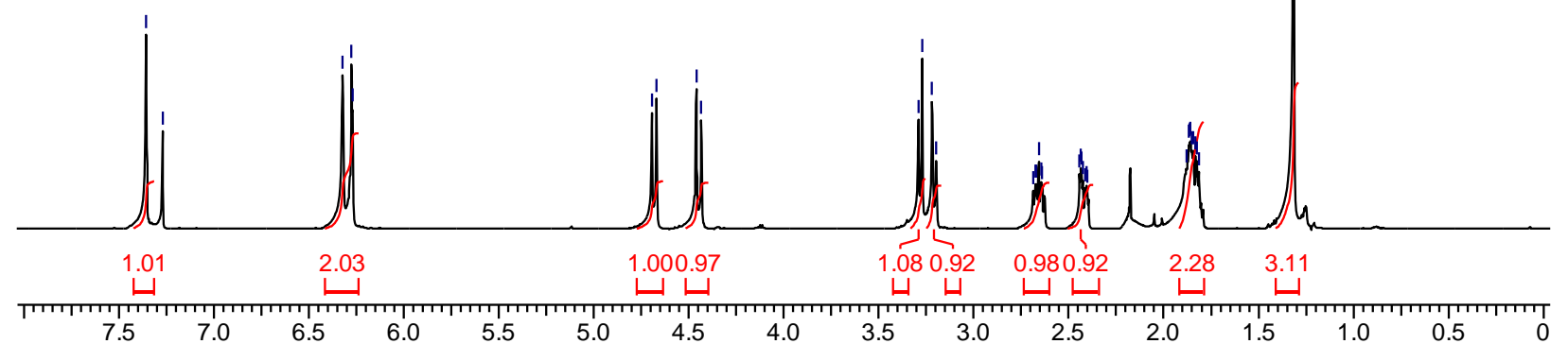

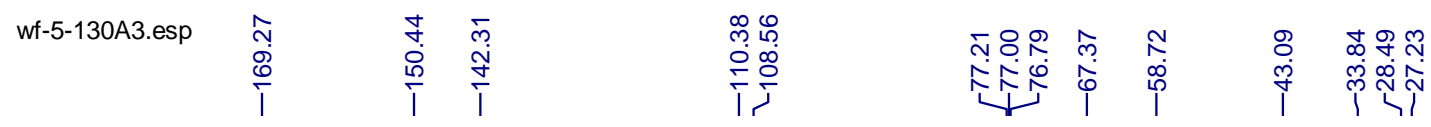

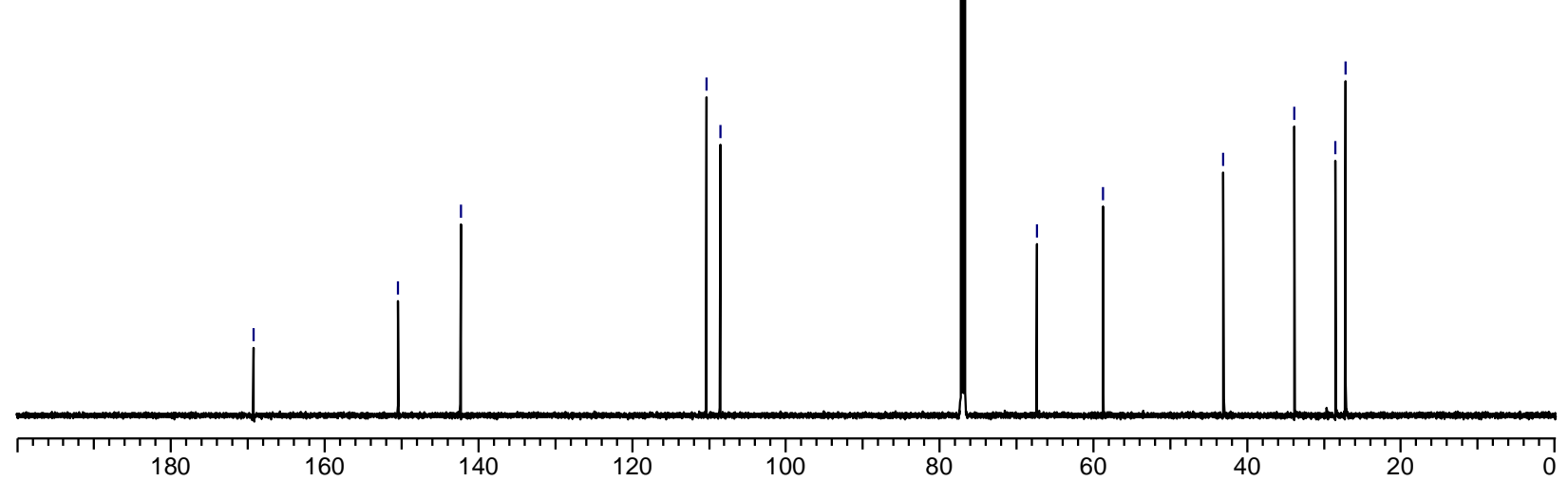




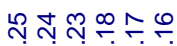

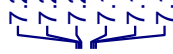

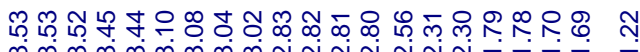

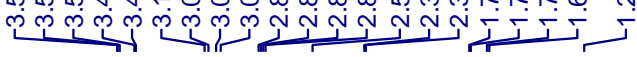<smiles>CC1(O)CCC(=O)N(CCc2ccccc2)C1</smiles>
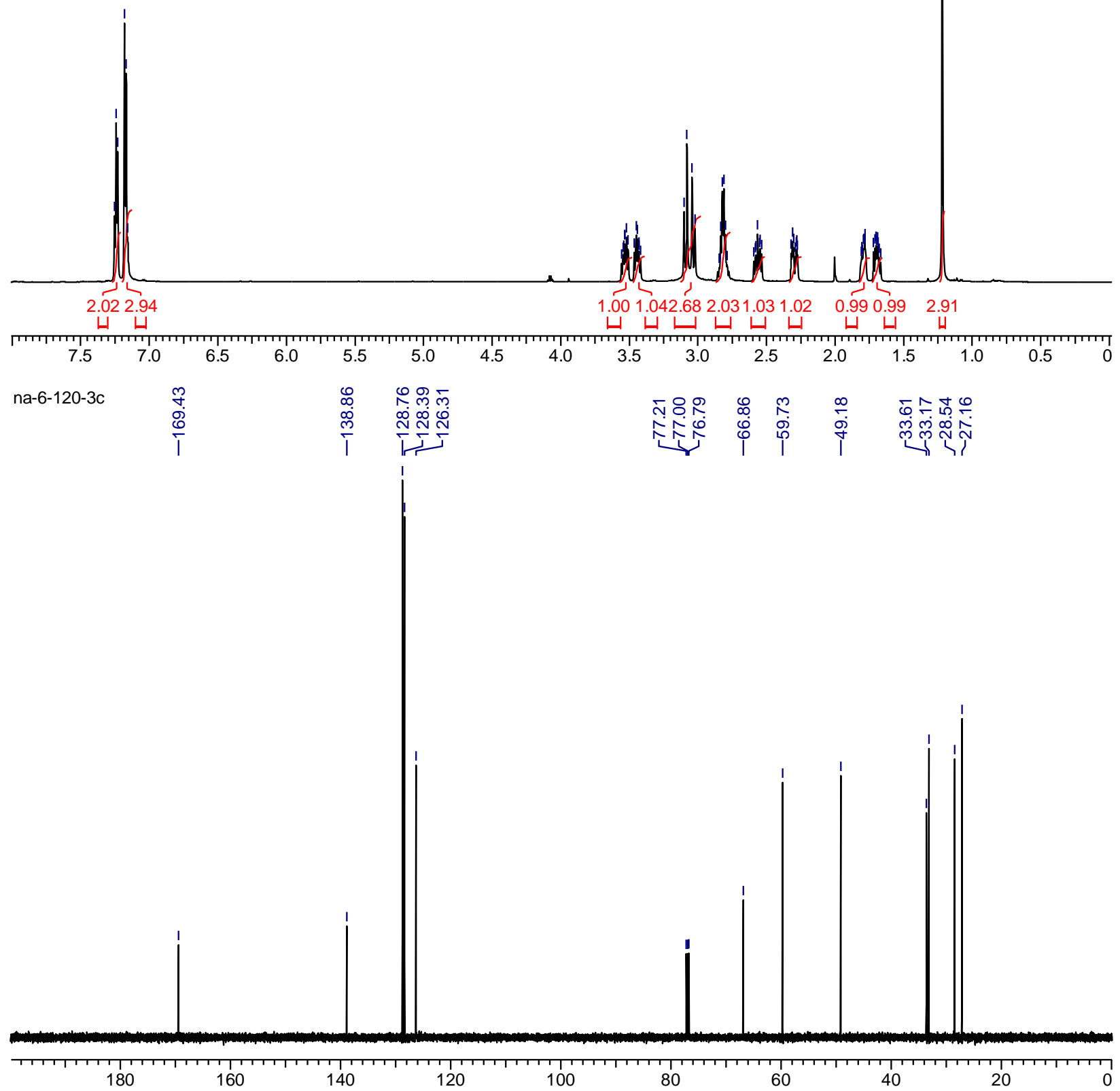
กิ่

选拄<smiles>CC1(O)CCC(=O)N(CCc2ccc(Cl)cc2)C1</smiles>

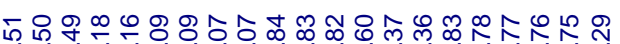

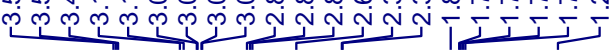

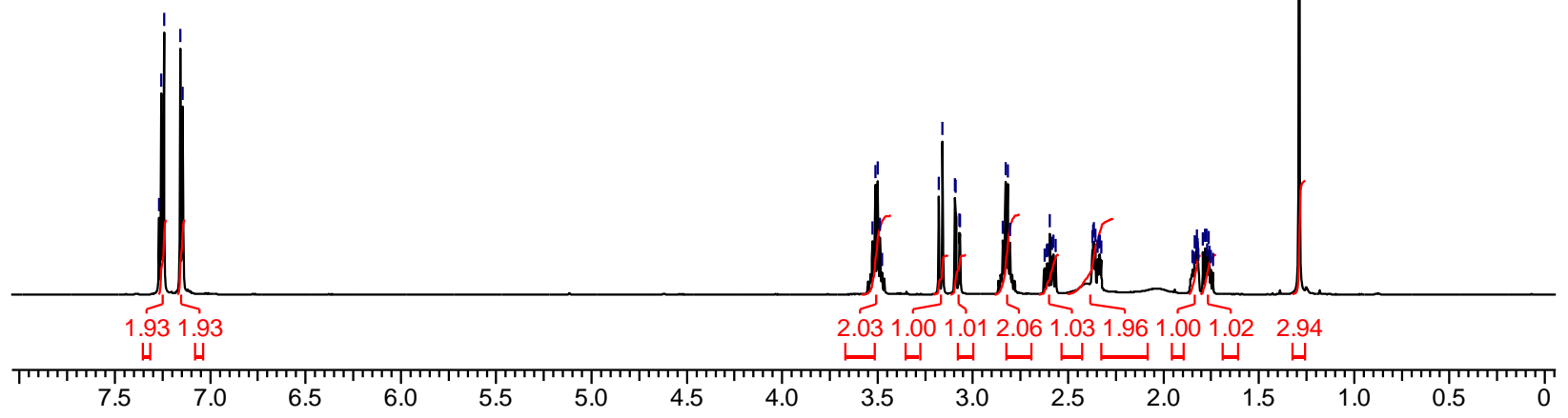

JA-9-C

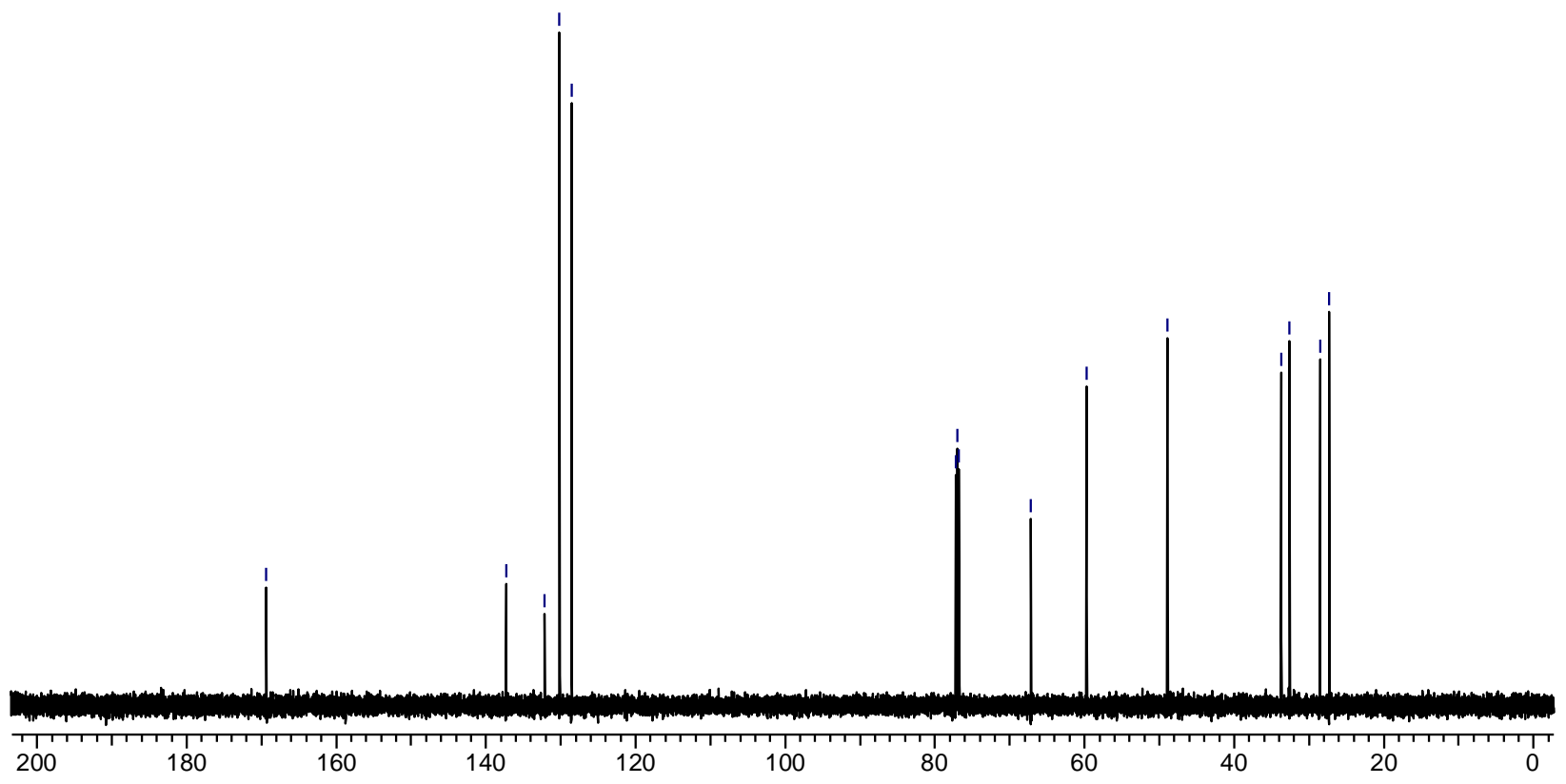




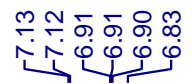<smiles>CC1(O)CCC(=O)N(CCc2cccs2)C1</smiles>

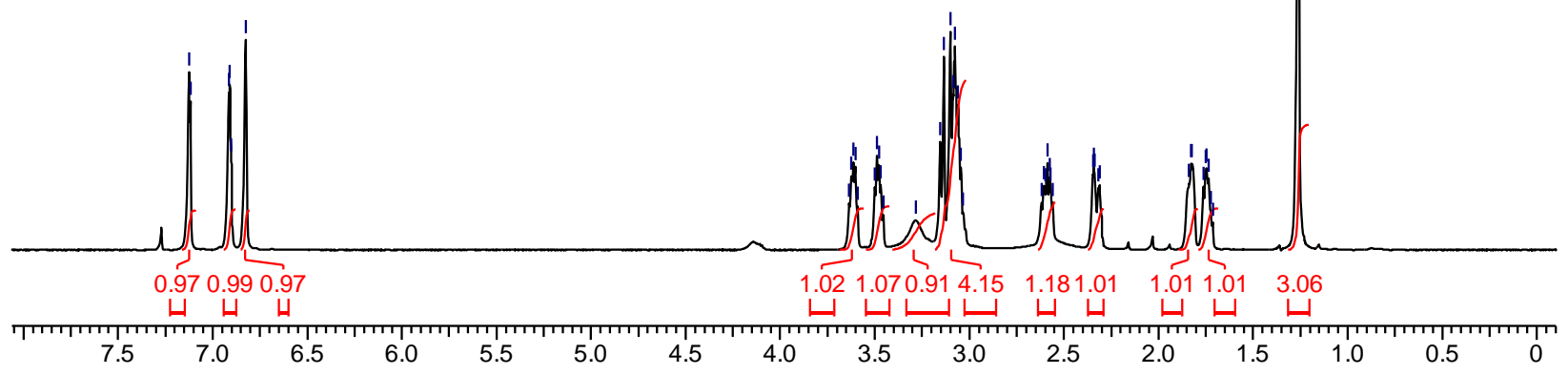

wf-5-130B313C

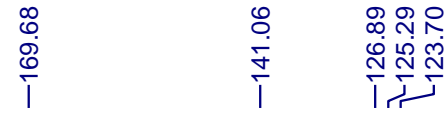

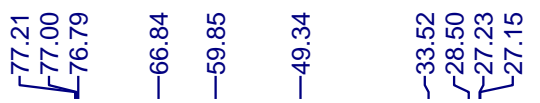

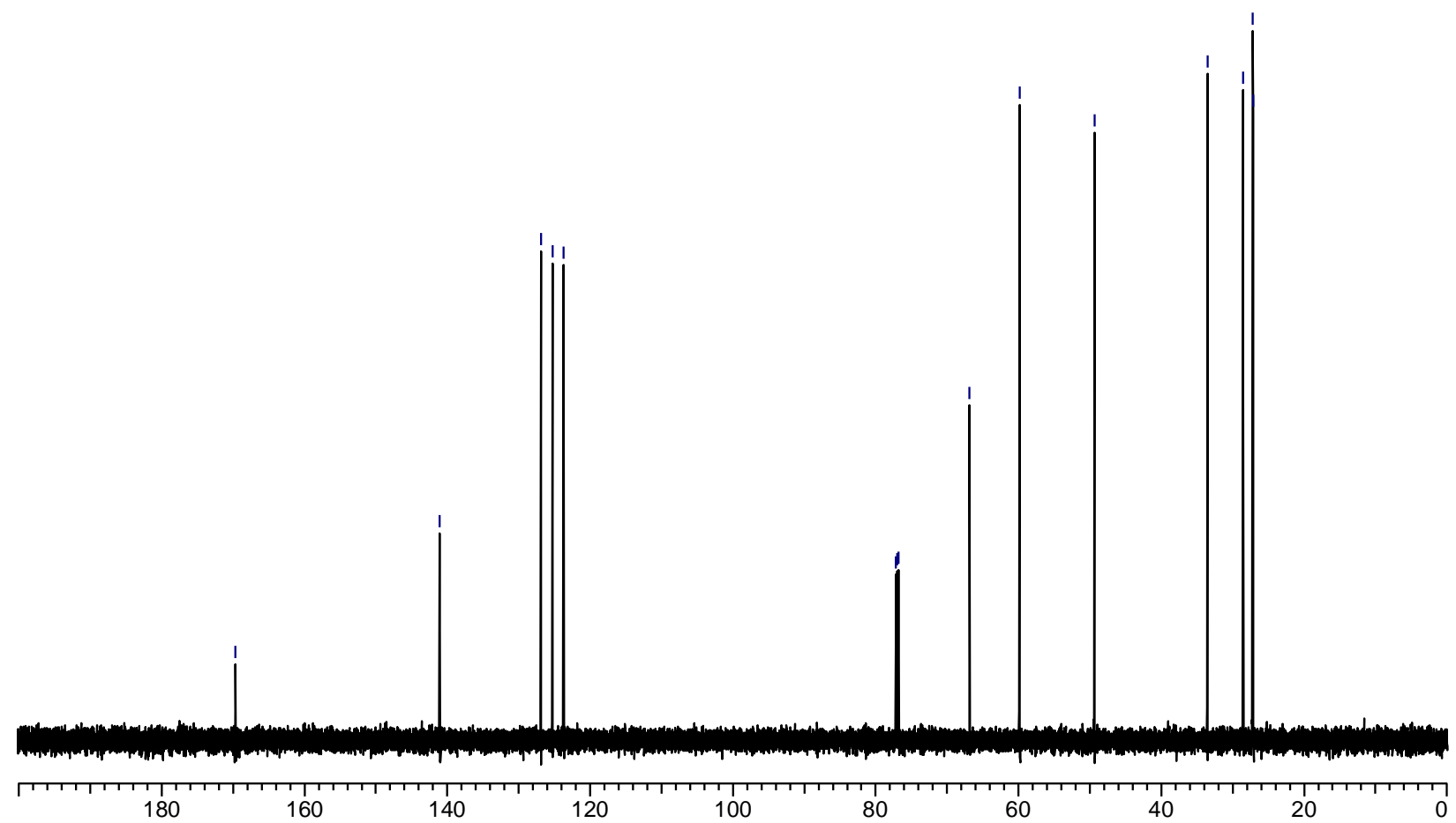


<smiles></smiles>

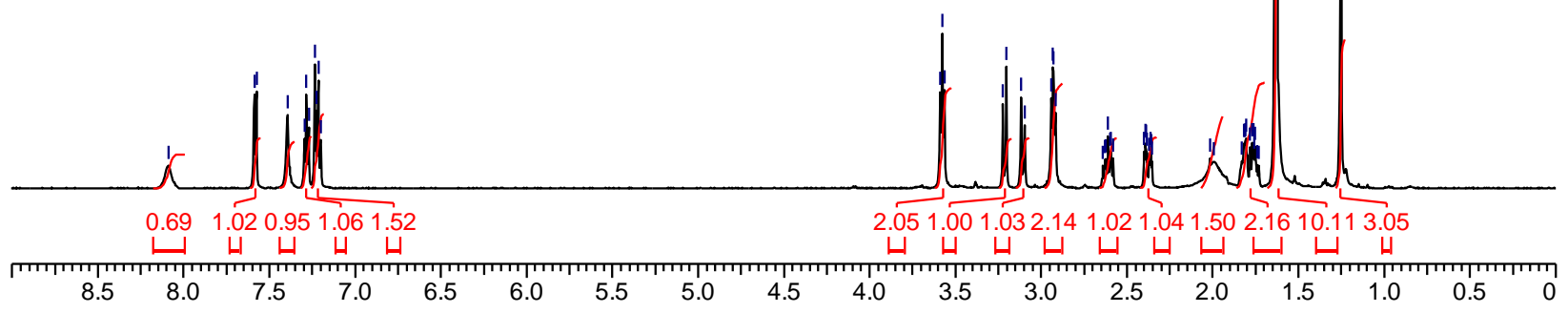

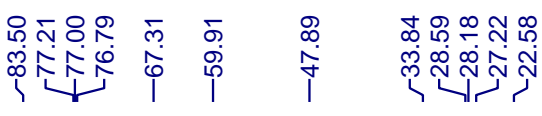

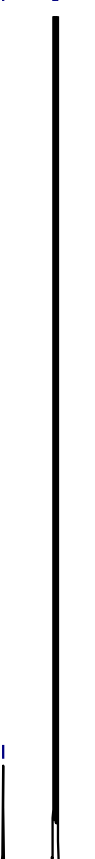




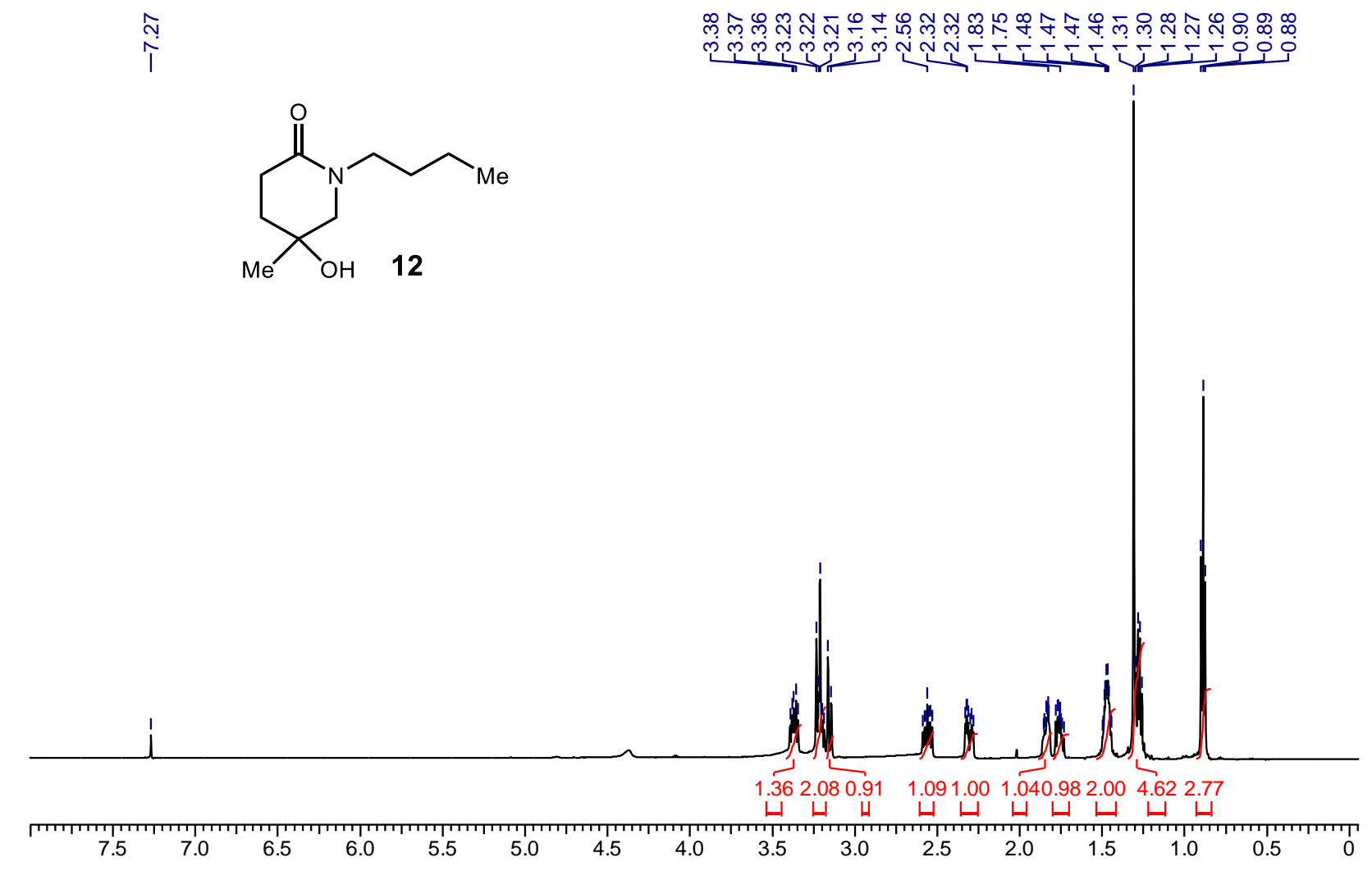

ஜ
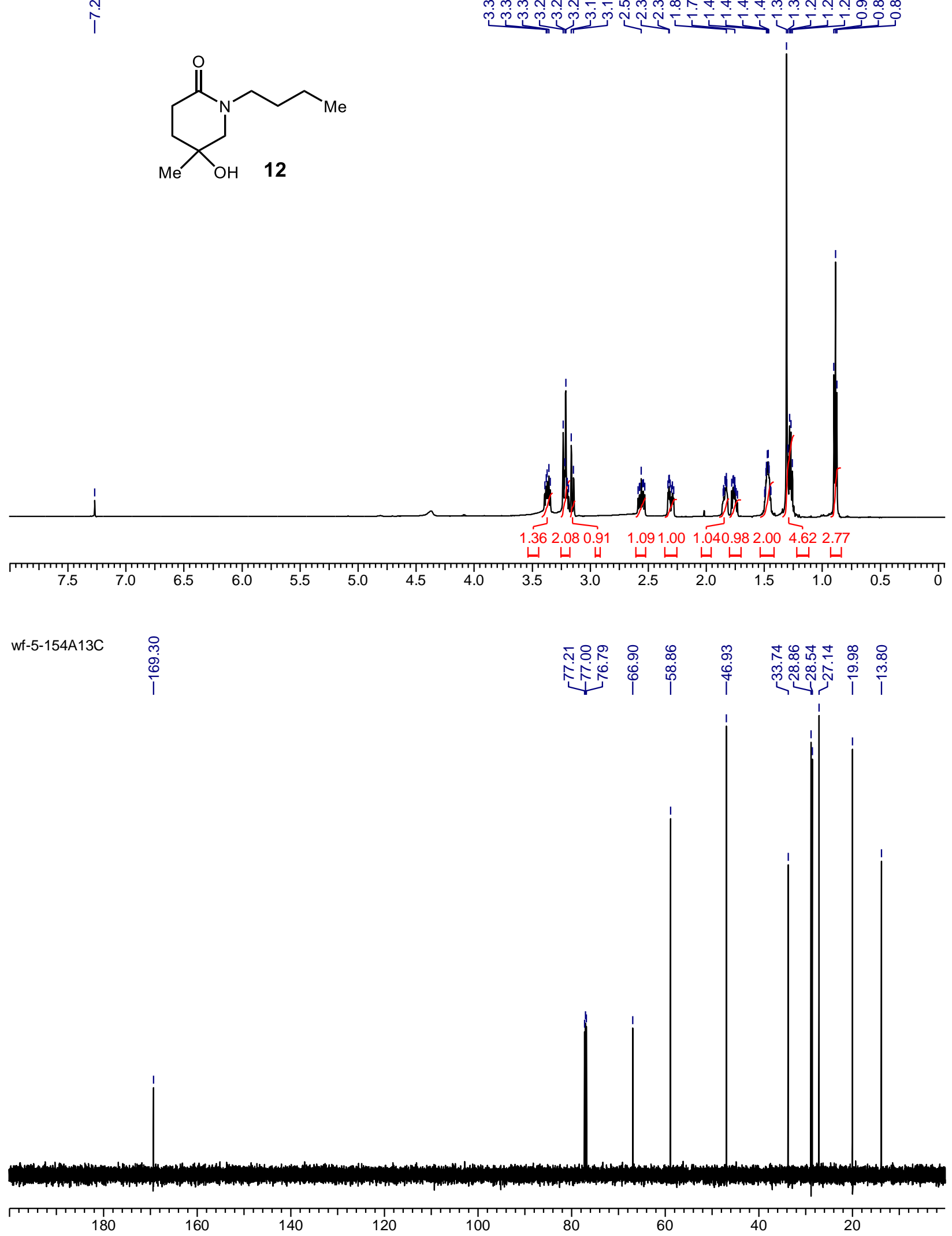
<smiles>CC(C)CCN1CC(C)(O)CCC1=O</smiles>
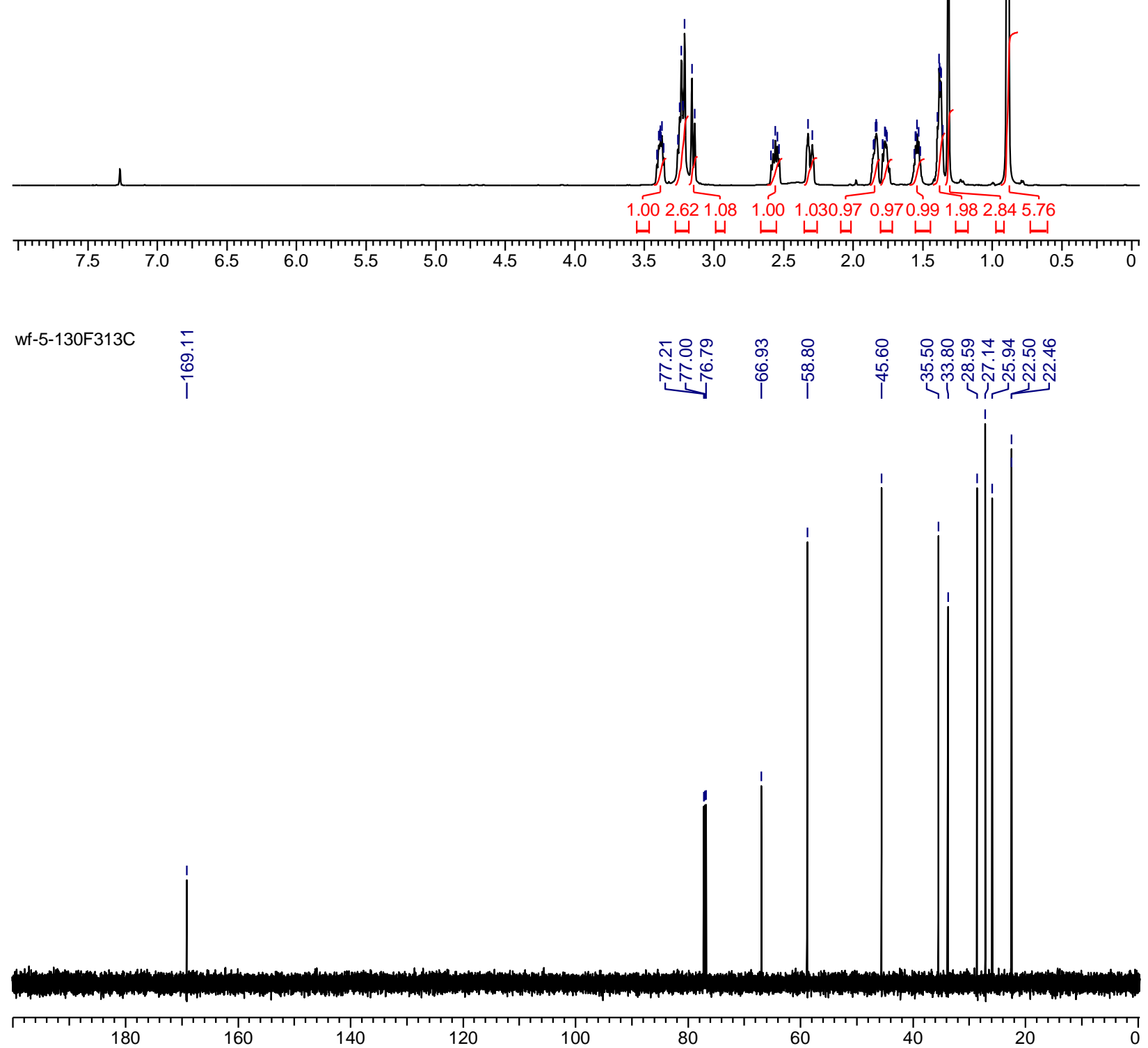
$\stackrel{\hat{N}}{\hat{\imath}}$

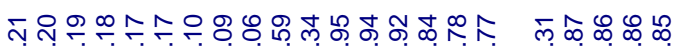

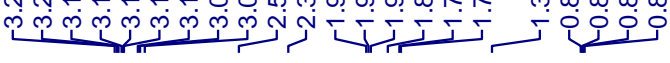<smiles>CC(C)CN1CC(C)(O)CCC1=O</smiles>

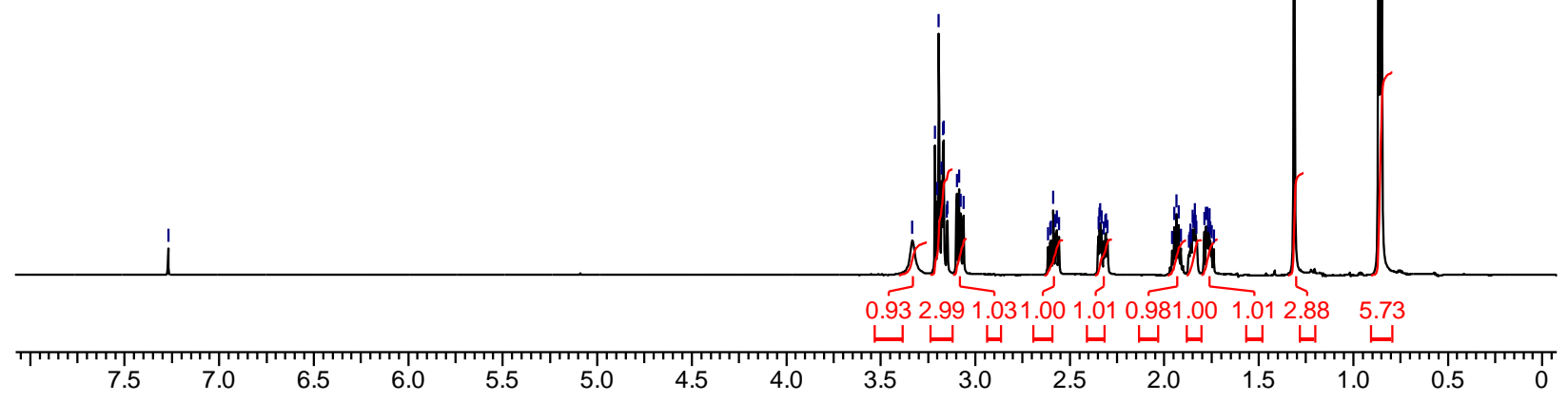

JA-13-C.001.esp

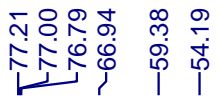

우웅

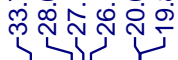

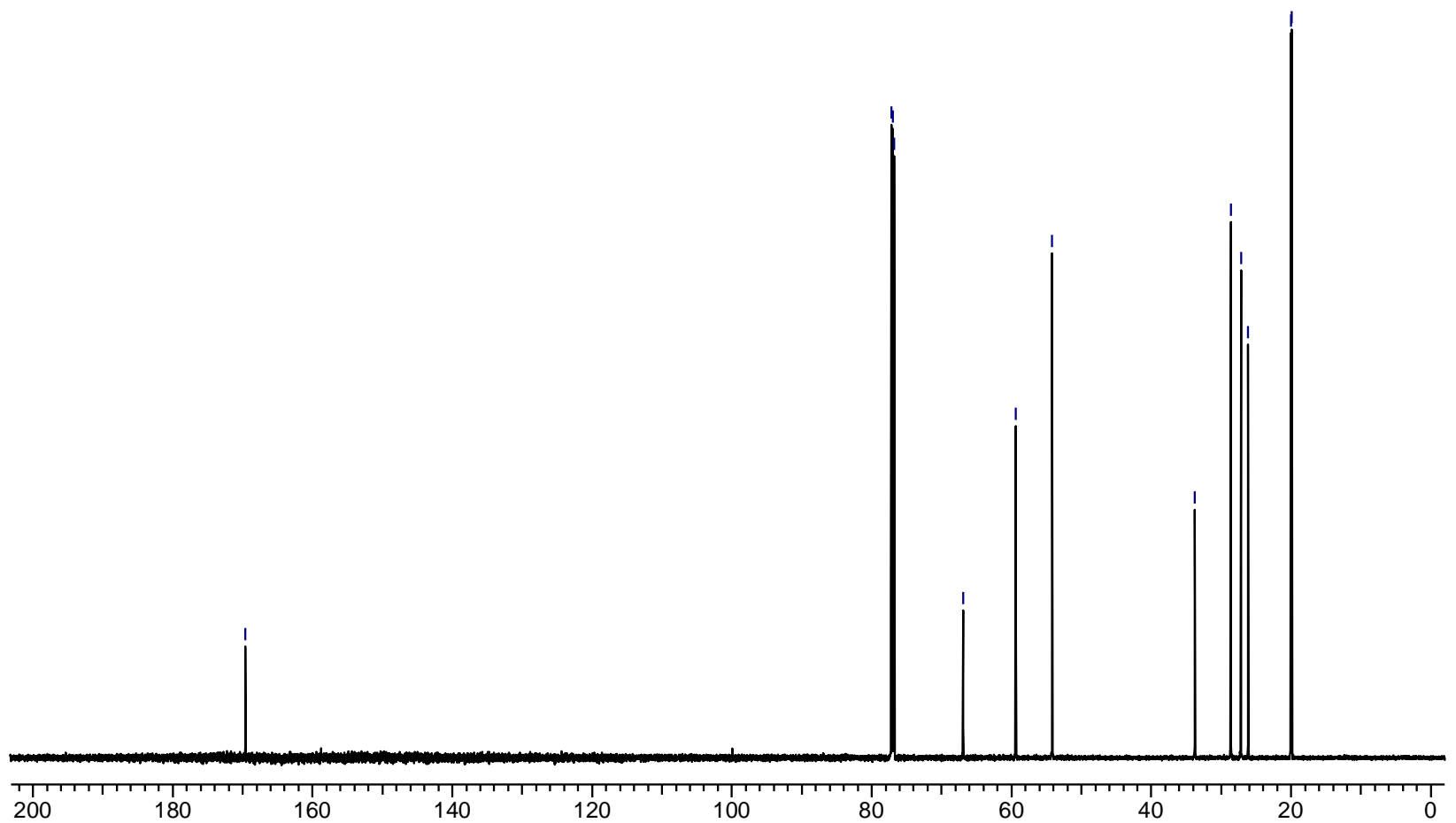




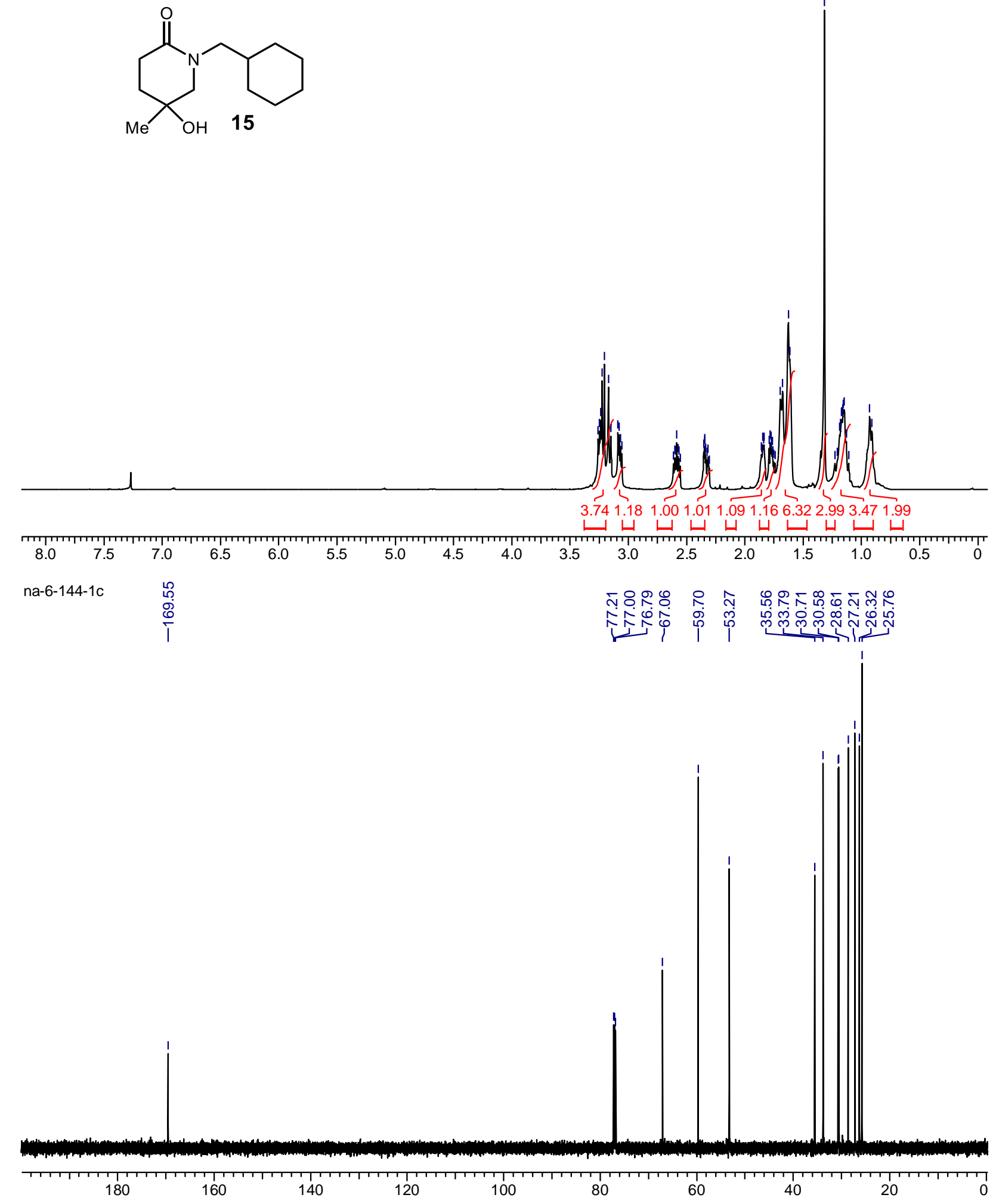


$\stackrel{\hat{N}}{\uparrow}$

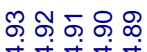

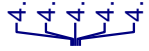

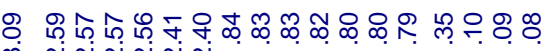

ن<smiles>CC(C)N1CC(C)(O)CCC1=O</smiles>

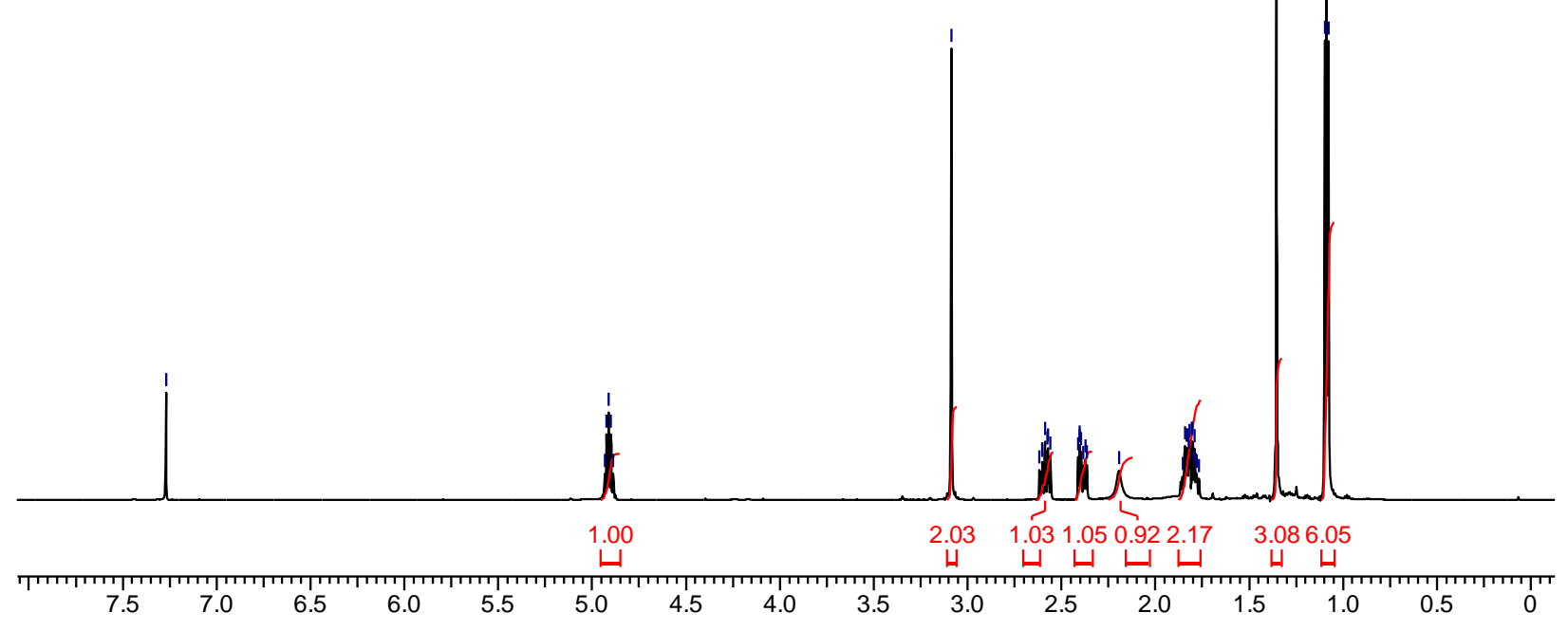

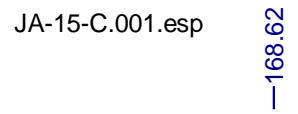

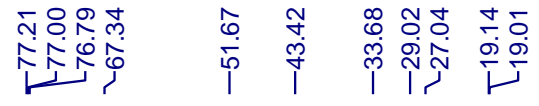

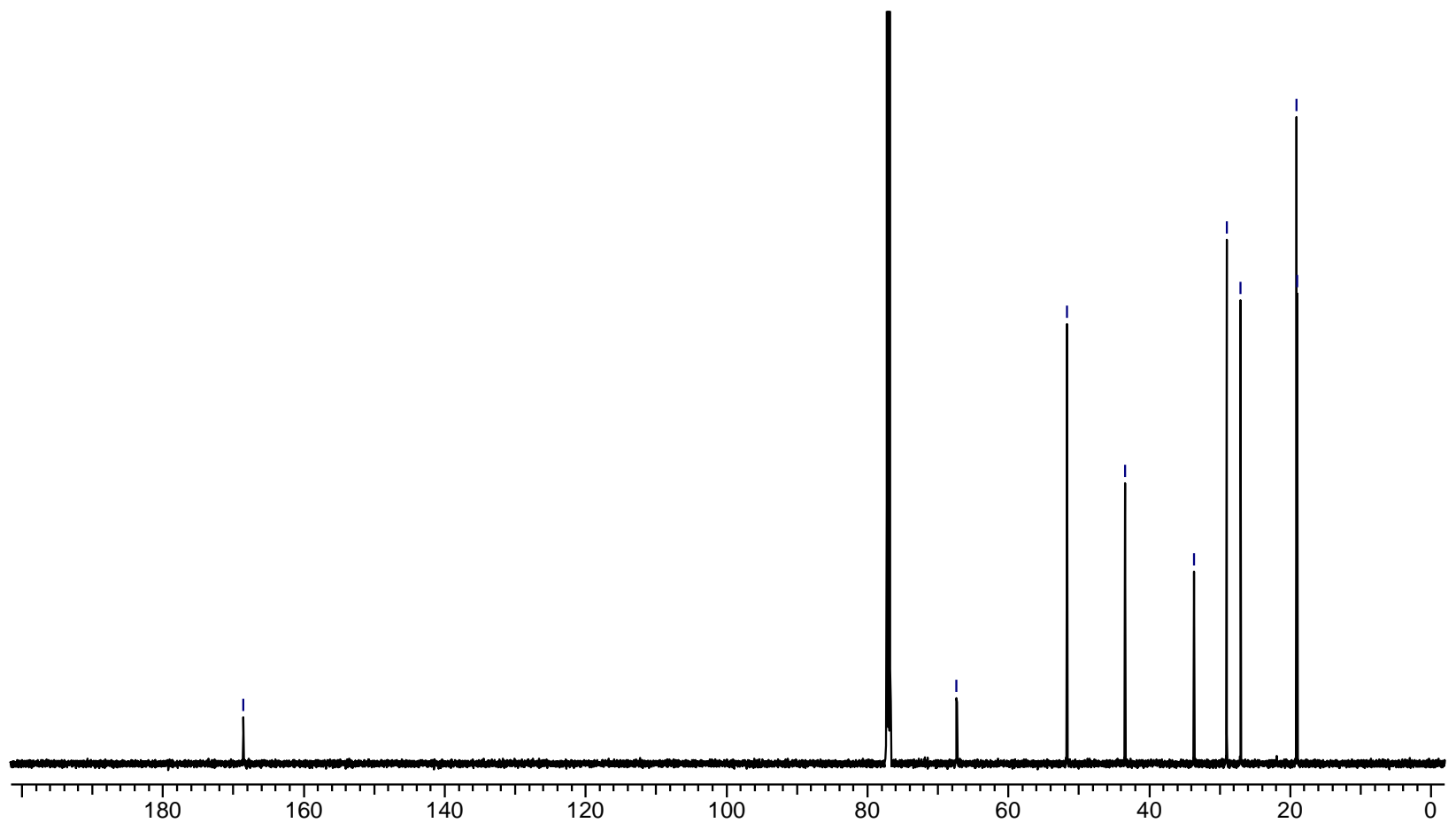


<smiles>CC1(O)CCC(=O)N(C2CCCC2)C1</smiles>

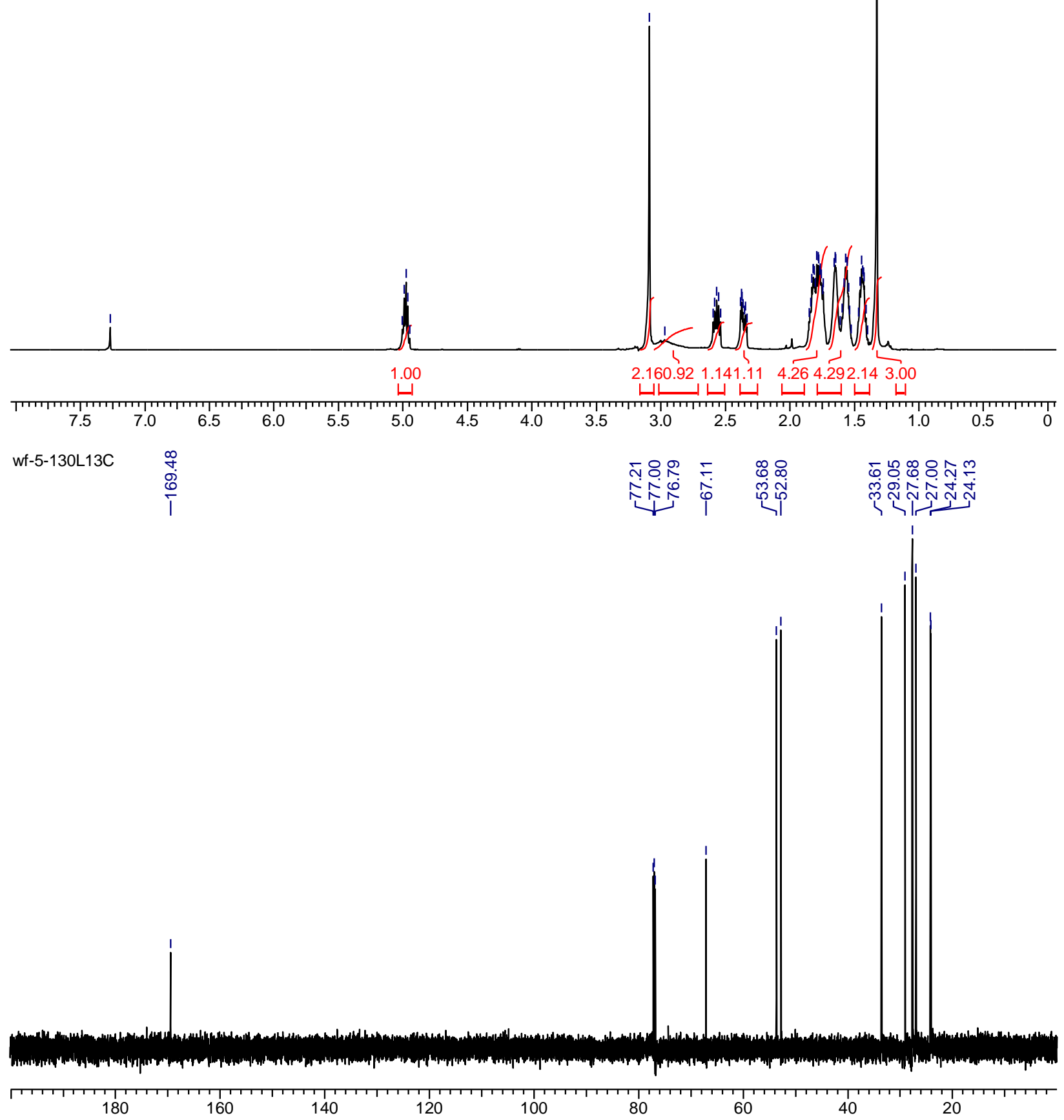



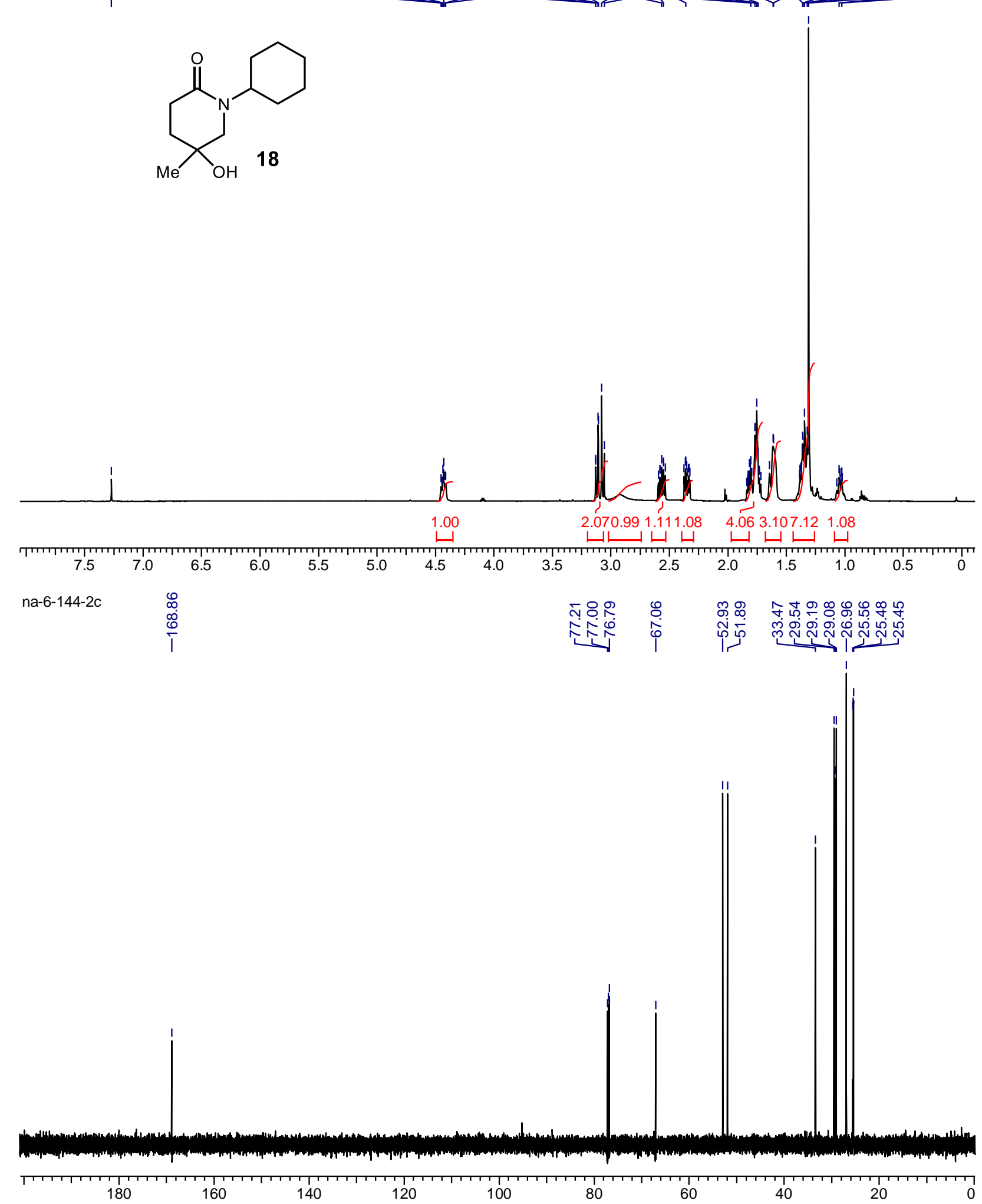
$\stackrel{\hat{N}}{\hat{i}}$<smiles>CC1(O)CCC(=O)N(C2CCCCCC2)C1</smiles>
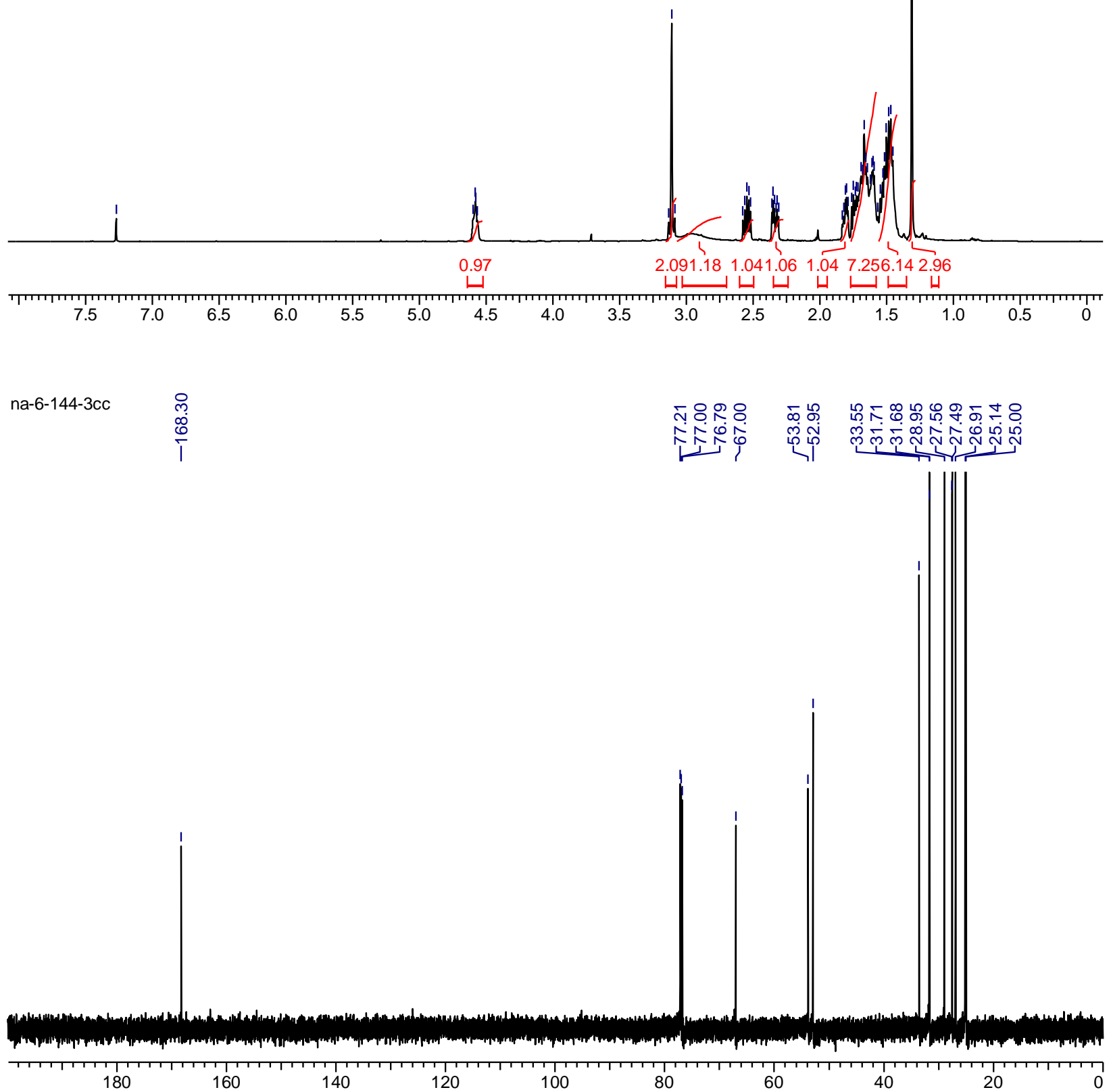
<smiles>O=C1CCC(O)(c2ccccc2)CN1Cc1ccccc1</smiles>
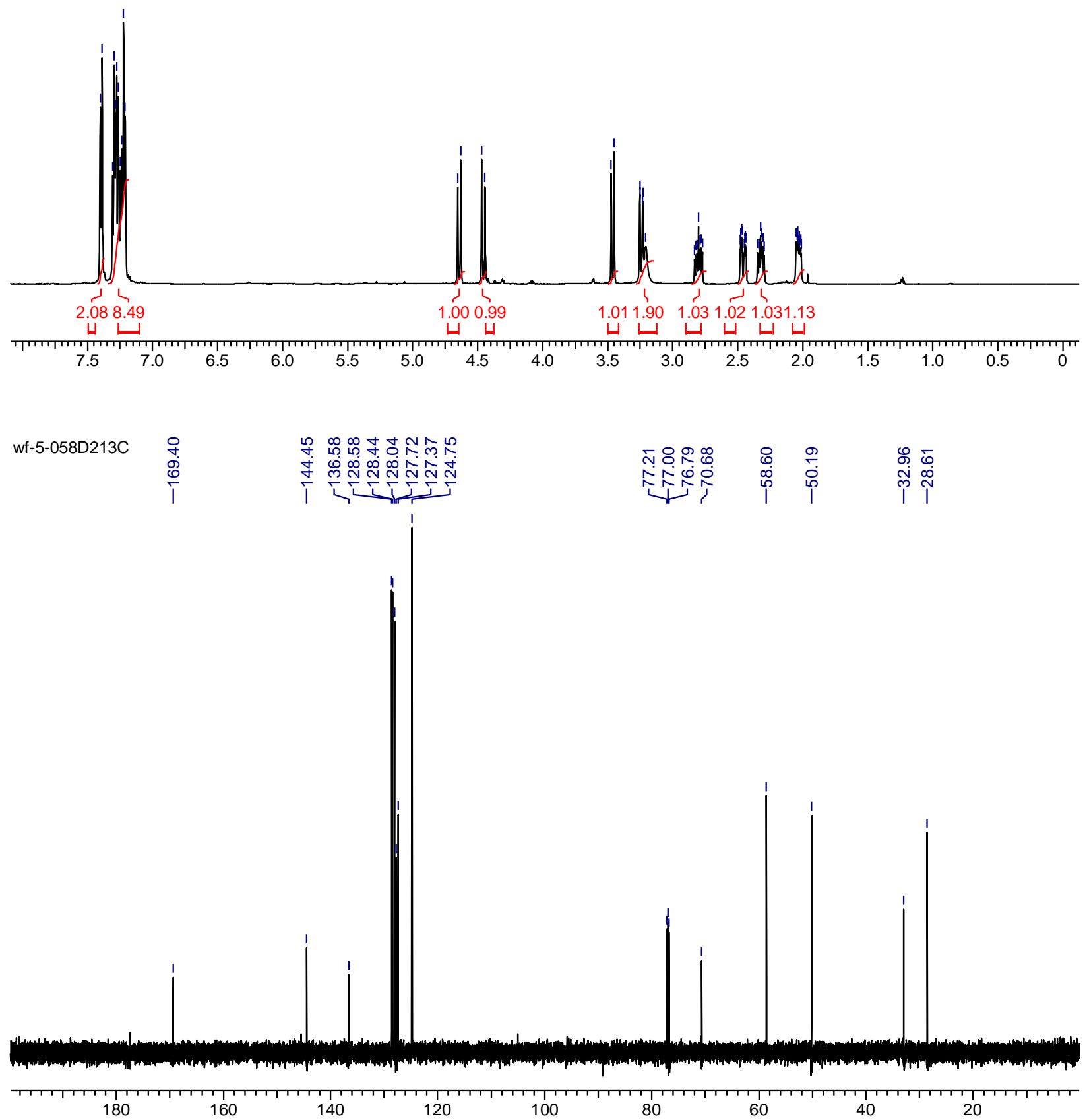
<smiles>O=C1CCC(O)(c2ccc(F)cc2)CN1Cc1ccccc1</smiles>

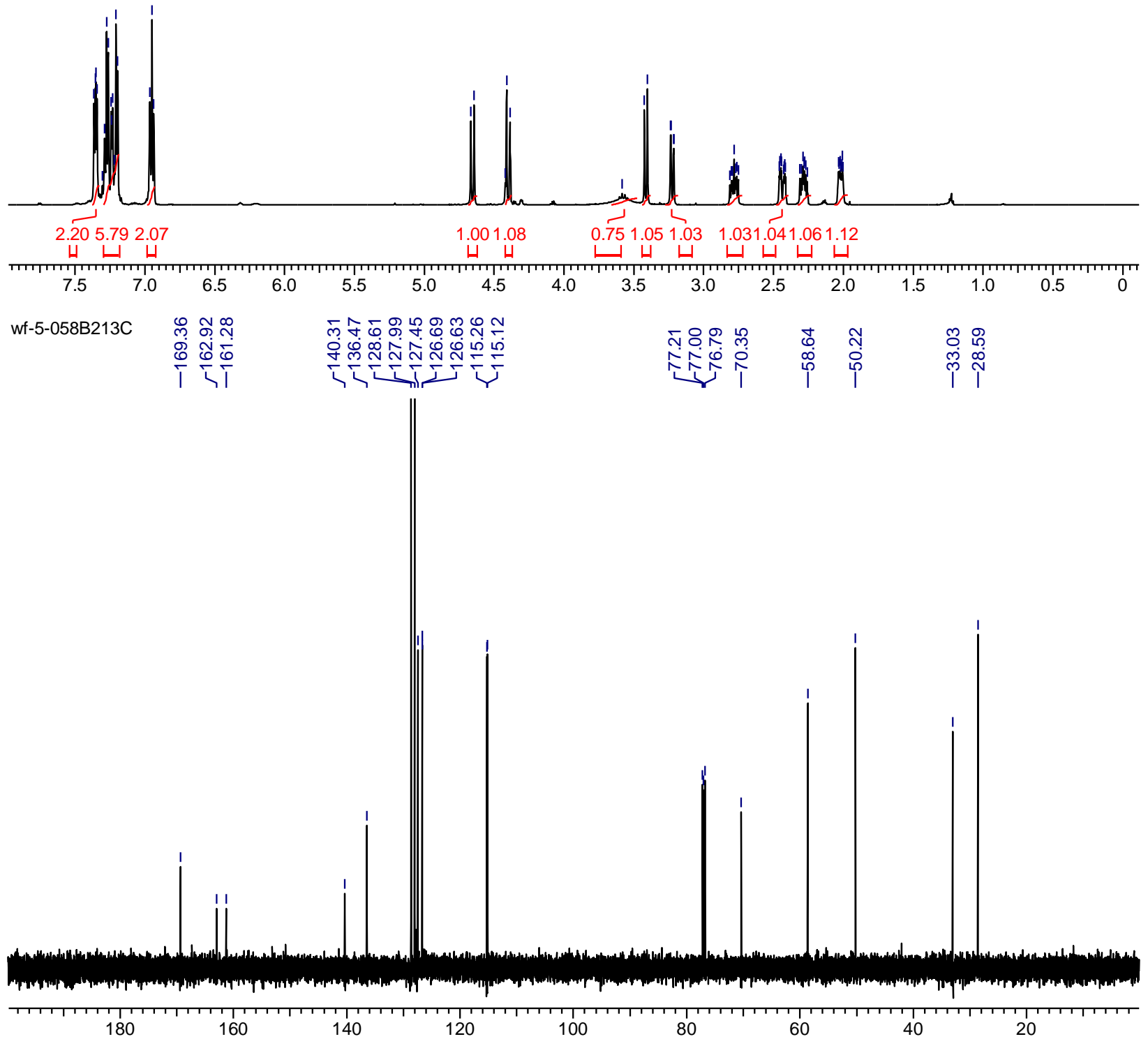


<smiles>O=C1CCC(O)(c2ccc(Cl)cc2)CN1Cc1ccccc1</smiles>

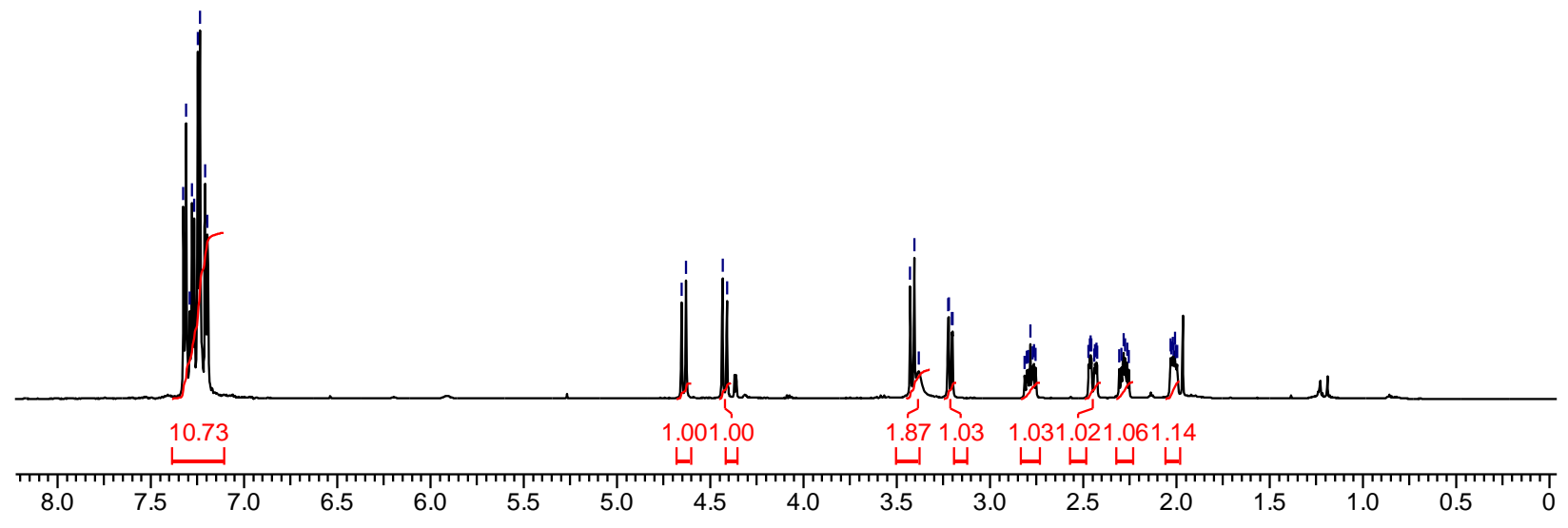

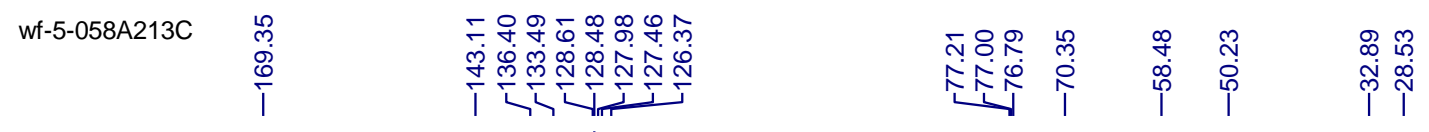

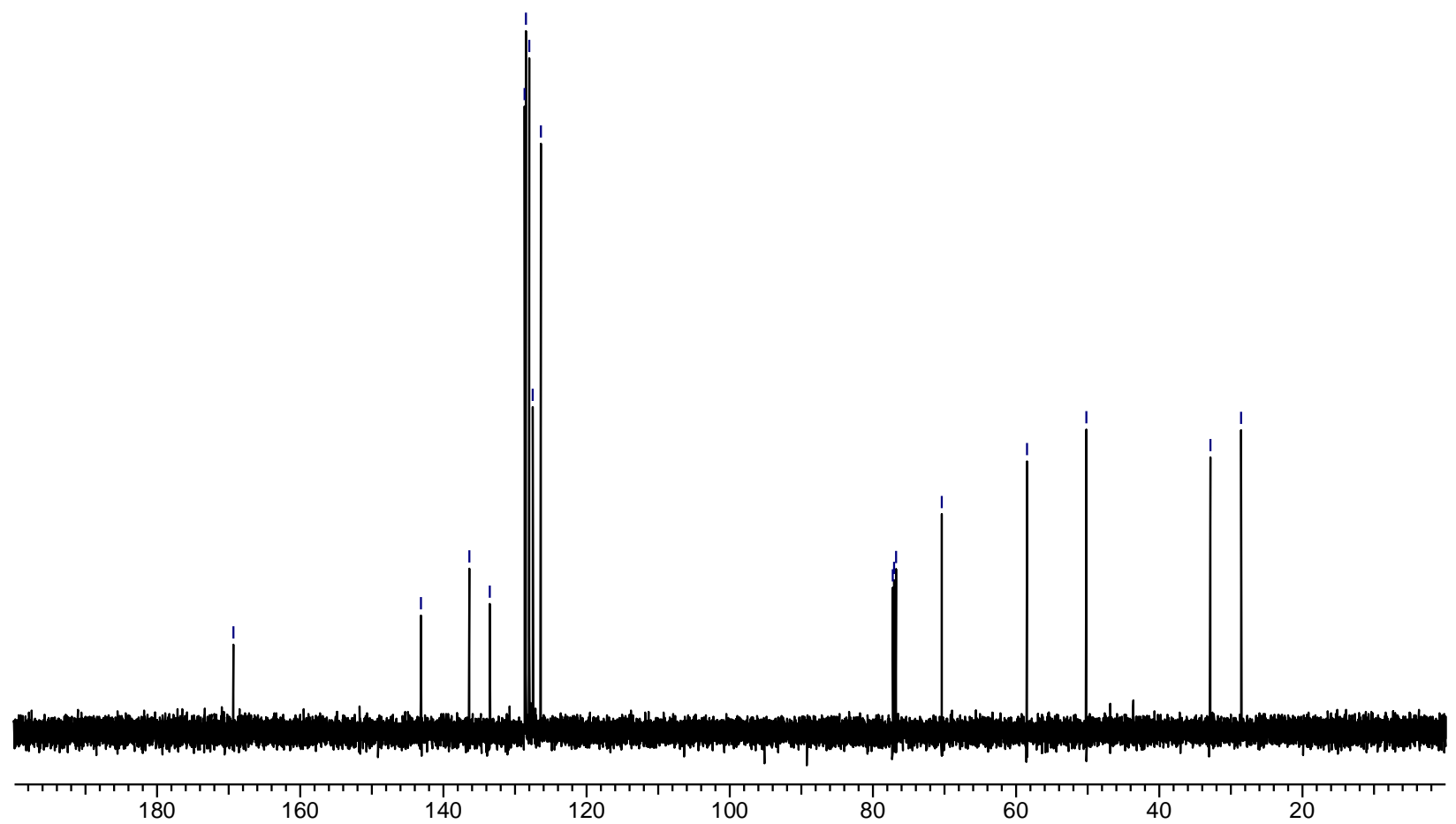




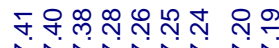 \\ Nisinis}

๒

落<smiles>O=C1CCC(O)(c2ccc(Br)cc2)CN1Cc1ccccc1</smiles>

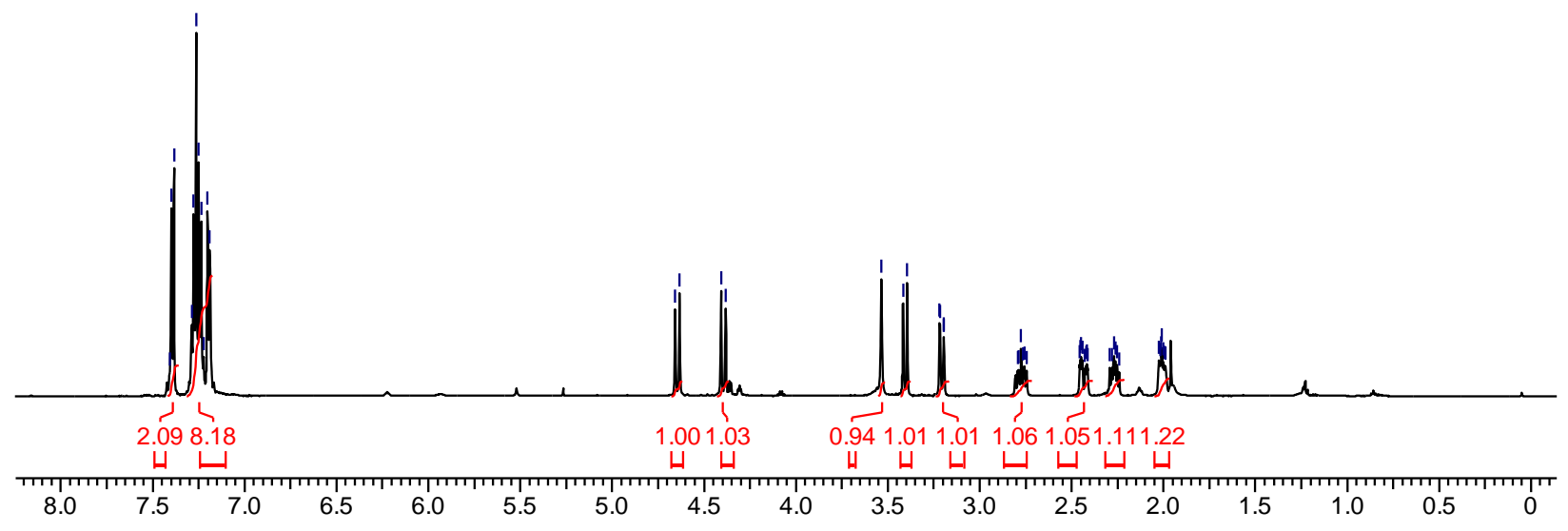

wf-5-130113C.001.es嘃

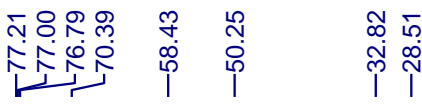

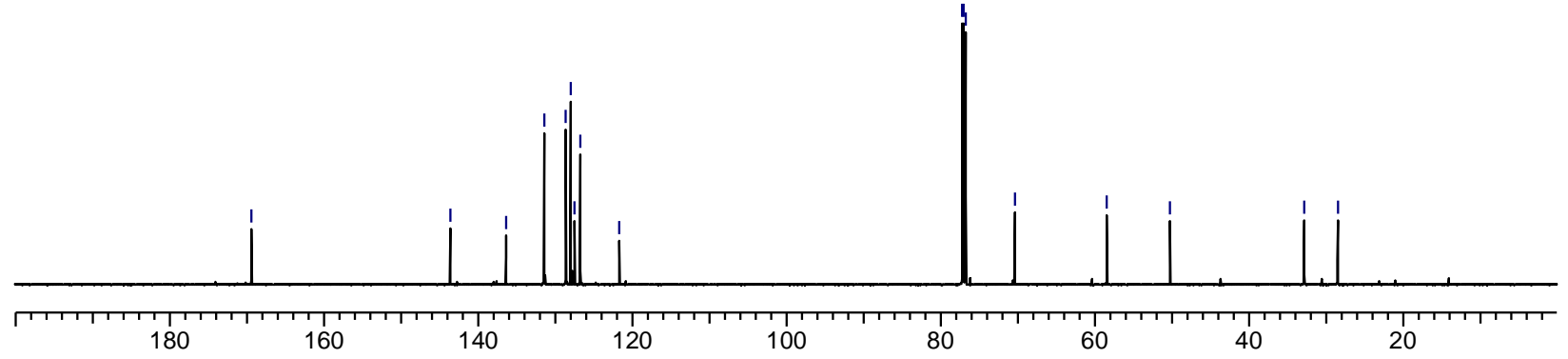




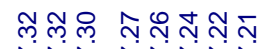

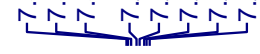

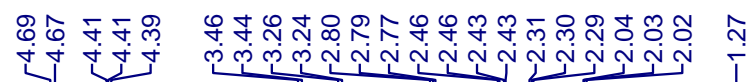<smiles>CC(C)(C)c1ccc(C2(O)CCC(=O)N(Cc3ccccc3)C2)cc1</smiles>
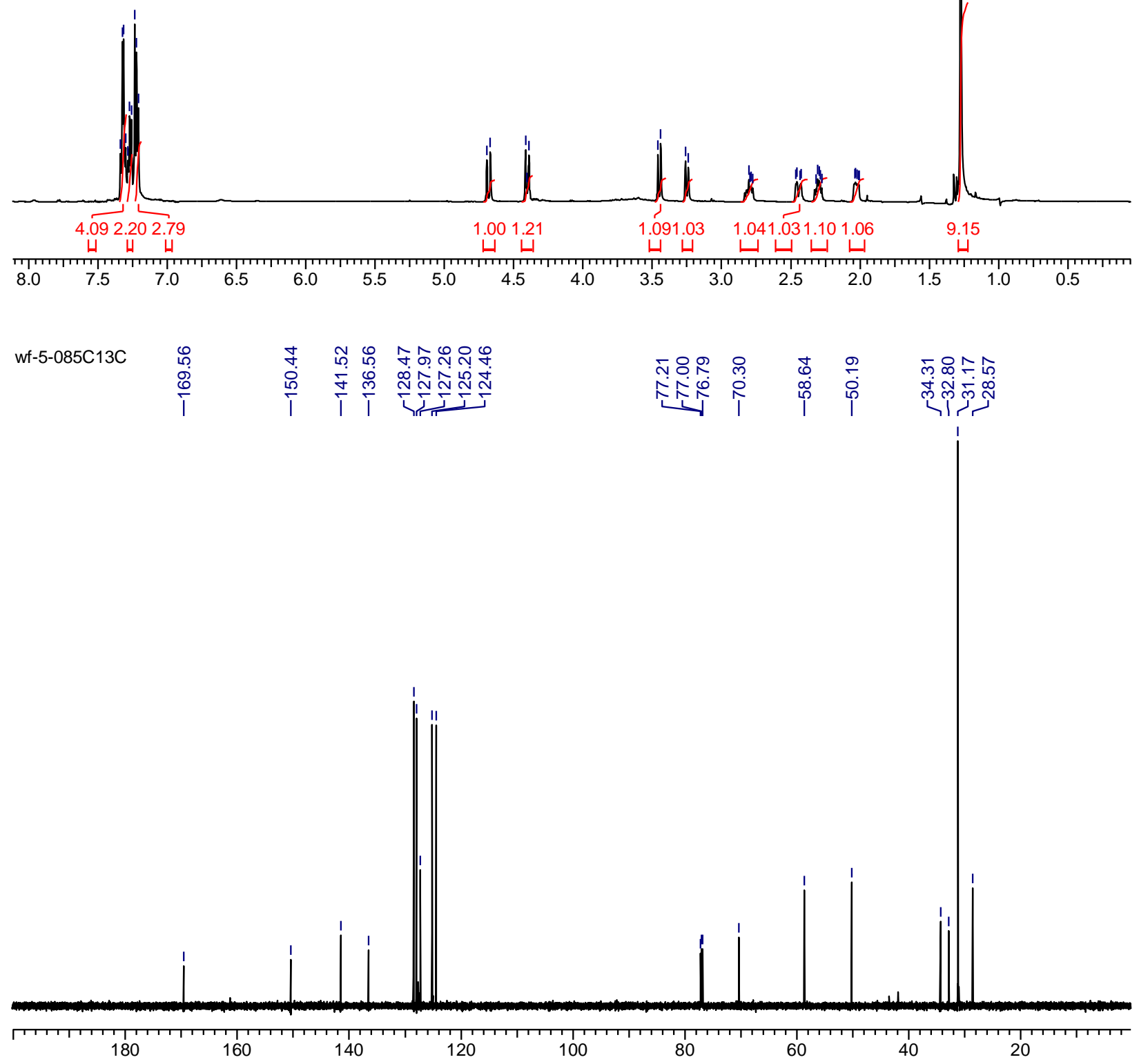
<smiles>O=C1CCC(O)(c2ccc(-c3ccccc3)cc2)CN1Cc1ccccc1</smiles>
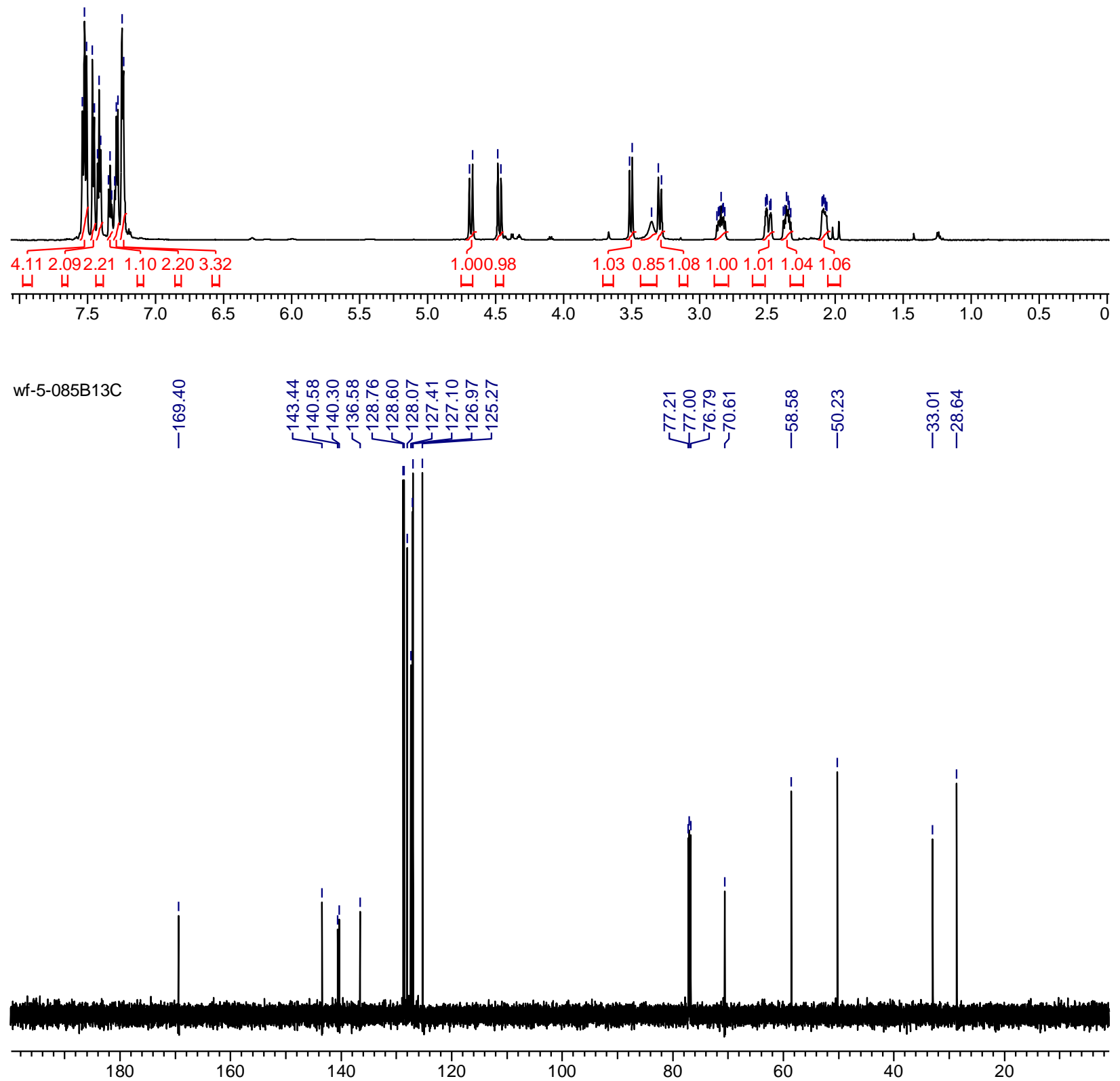
<smiles>O=C1CCC(O)CN1Cc1ccccc1</smiles>
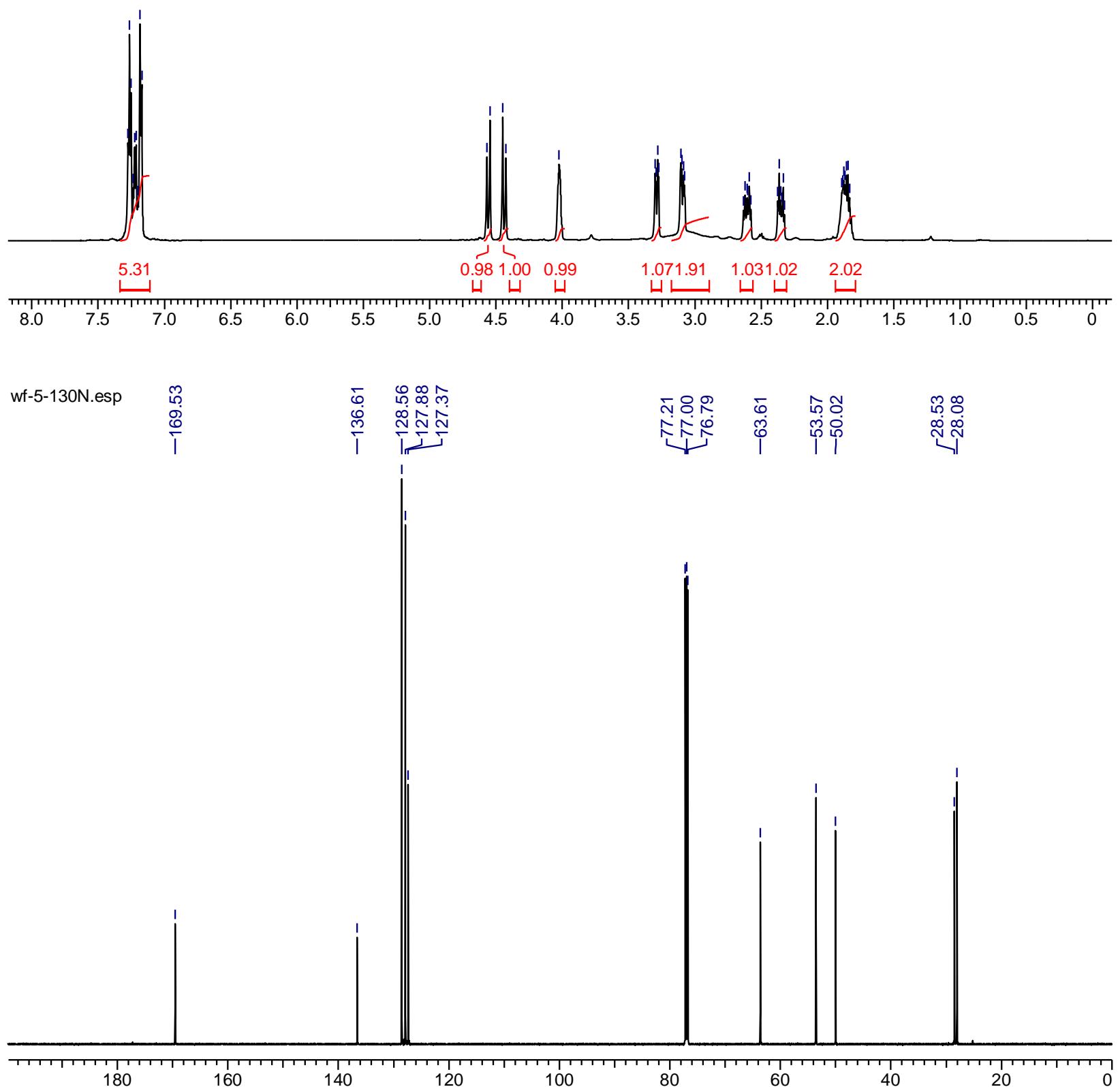
<smiles>O=C1CCC(O)(C2CC2)CN1Cc1ccccc1</smiles>
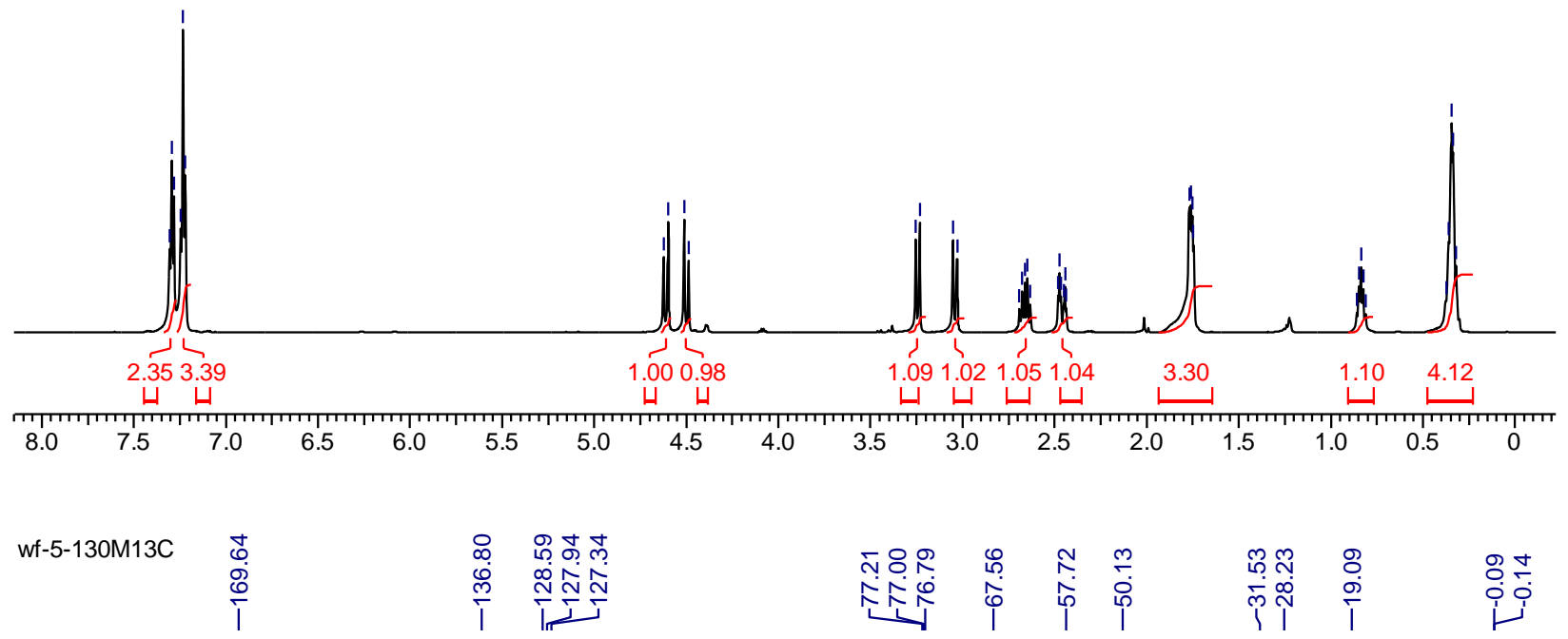

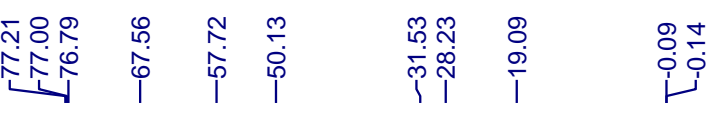

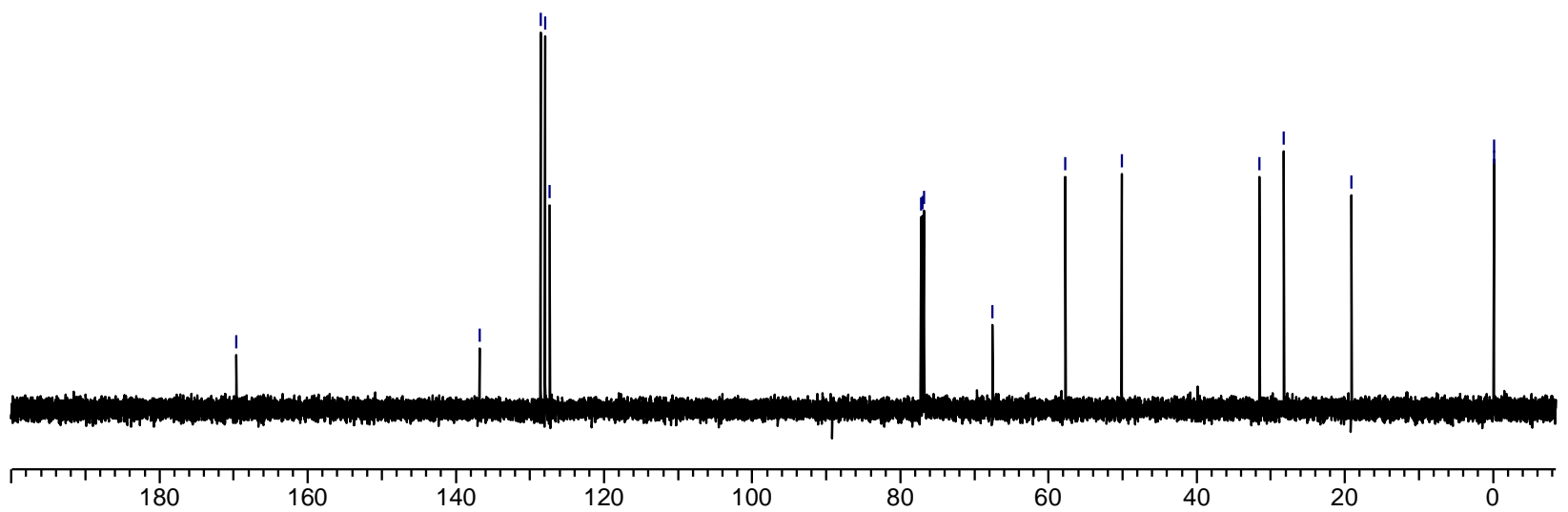


<smiles>CCCCN1CC(C)(O)c2ccccc2C1=O</smiles>
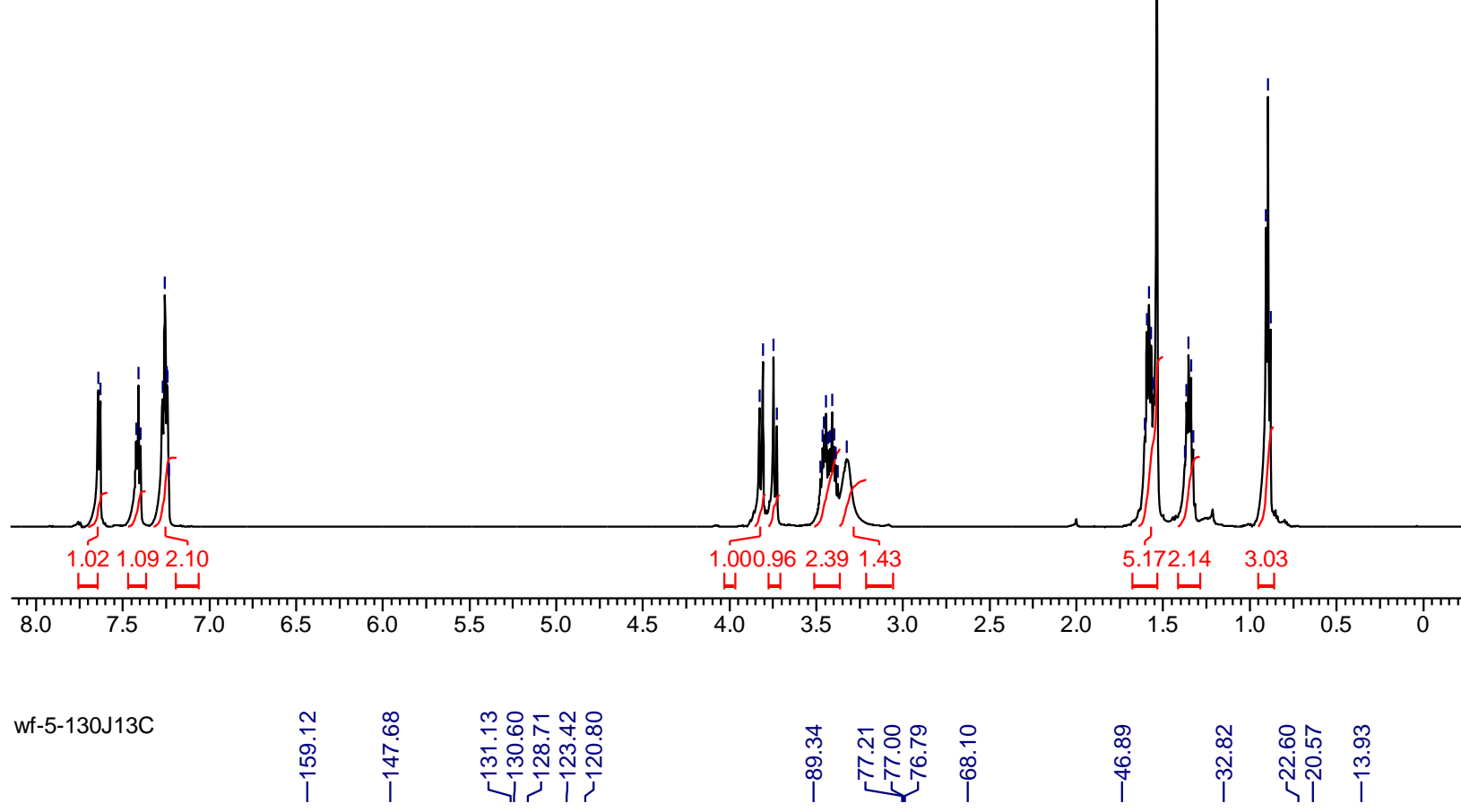

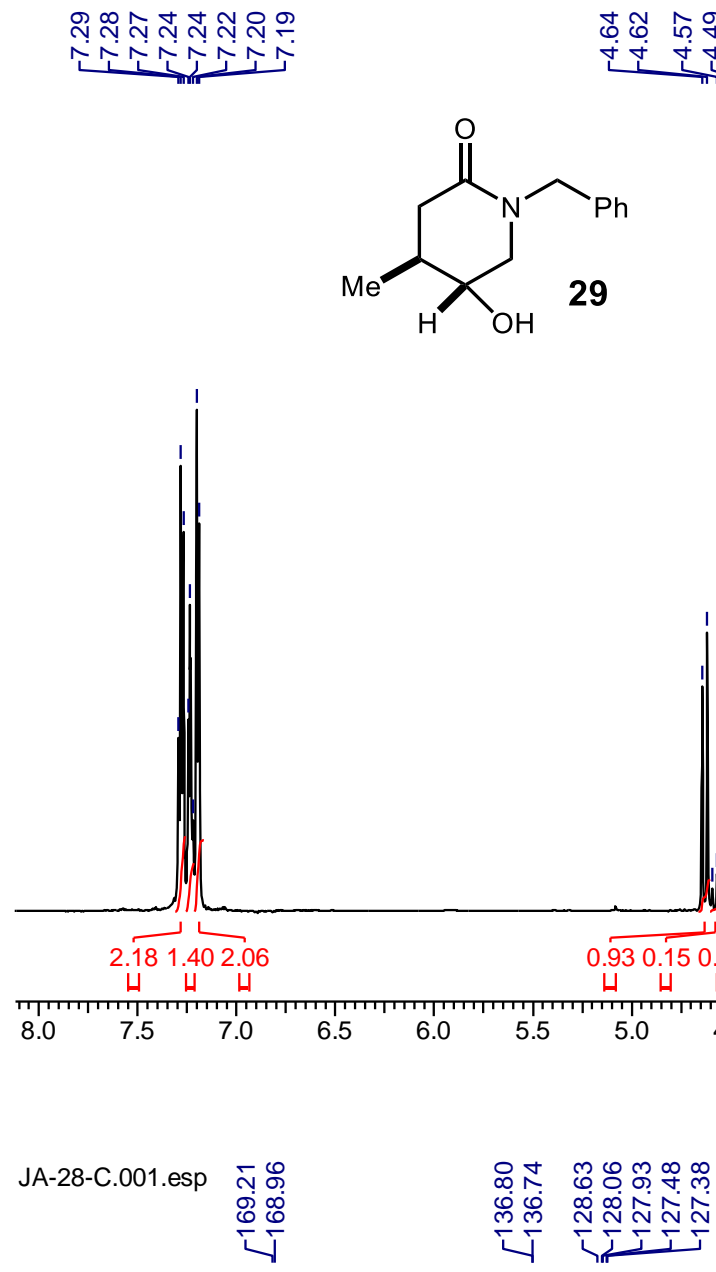

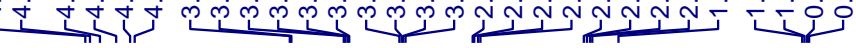
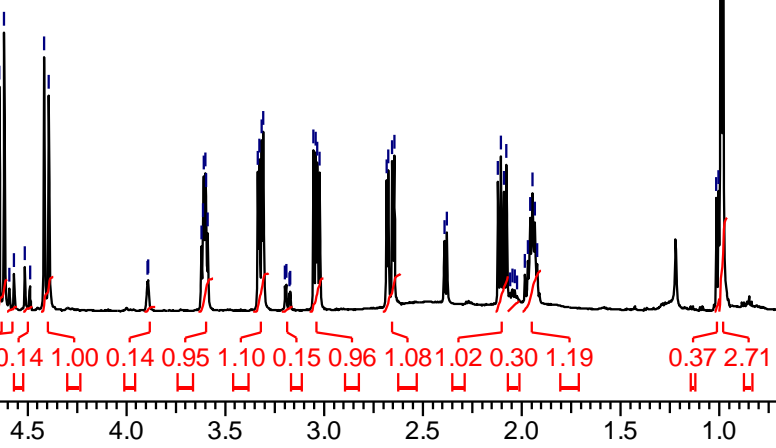

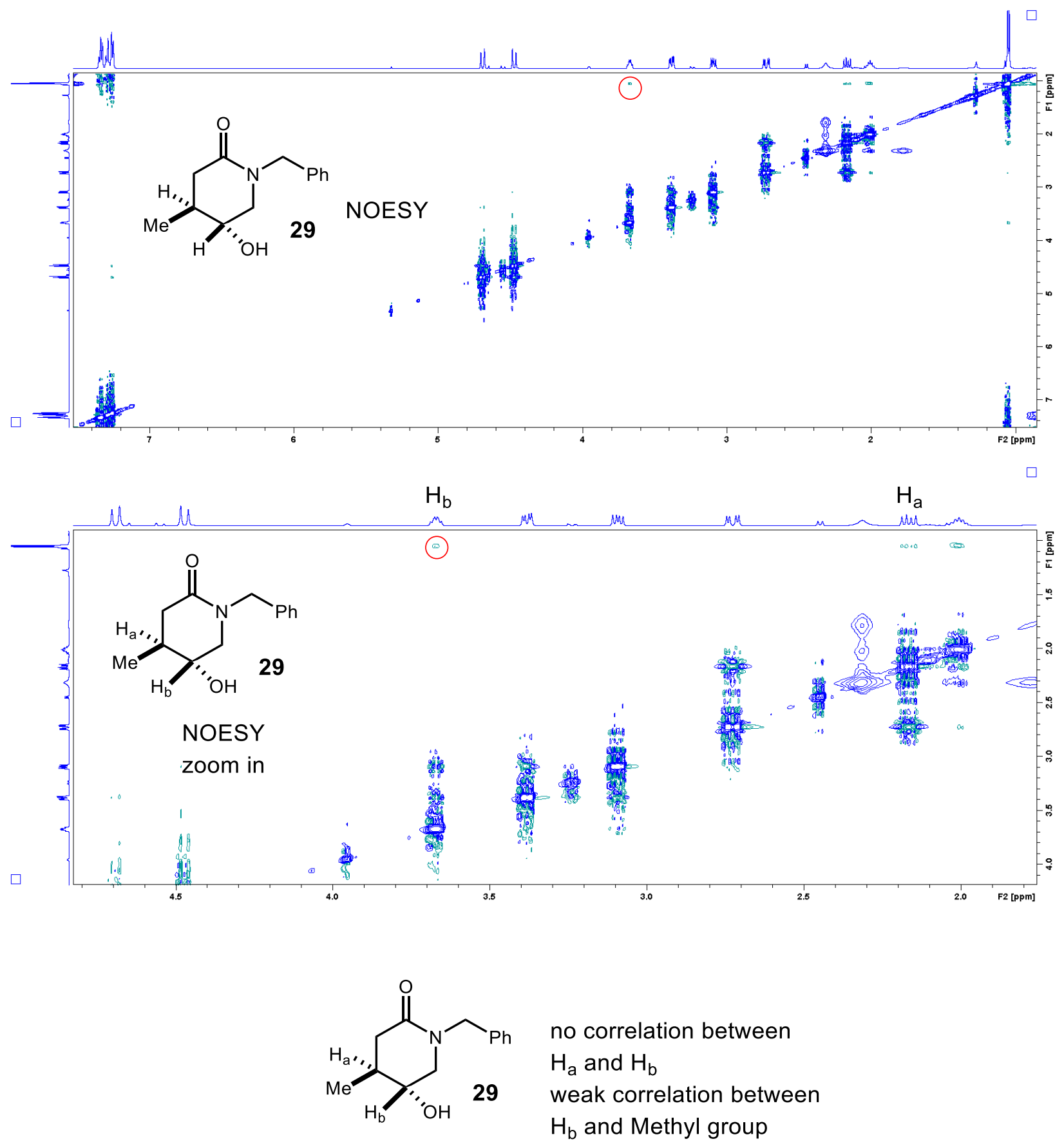
<smiles>O=C1CC(c2ccccc2)C(O)CN1Cc1ccccc1</smiles>

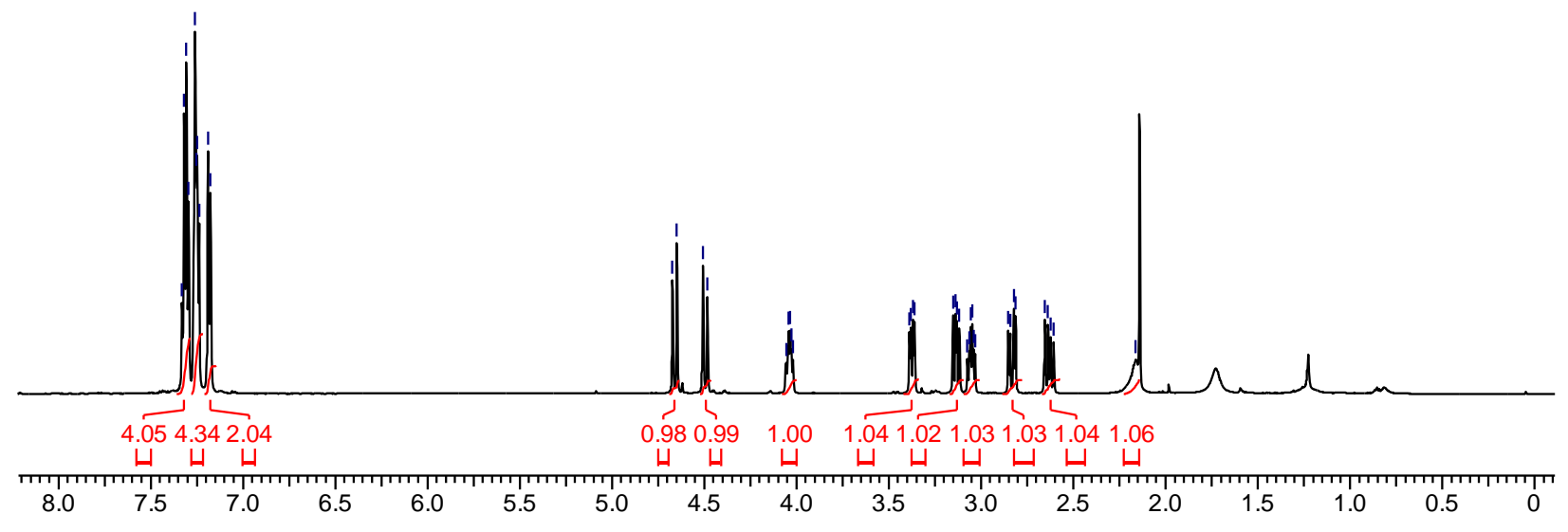

$\begin{array}{lll}\text { wf-5-1300.esp } & 0 & 0 \\ & 0 & 1\end{array}$
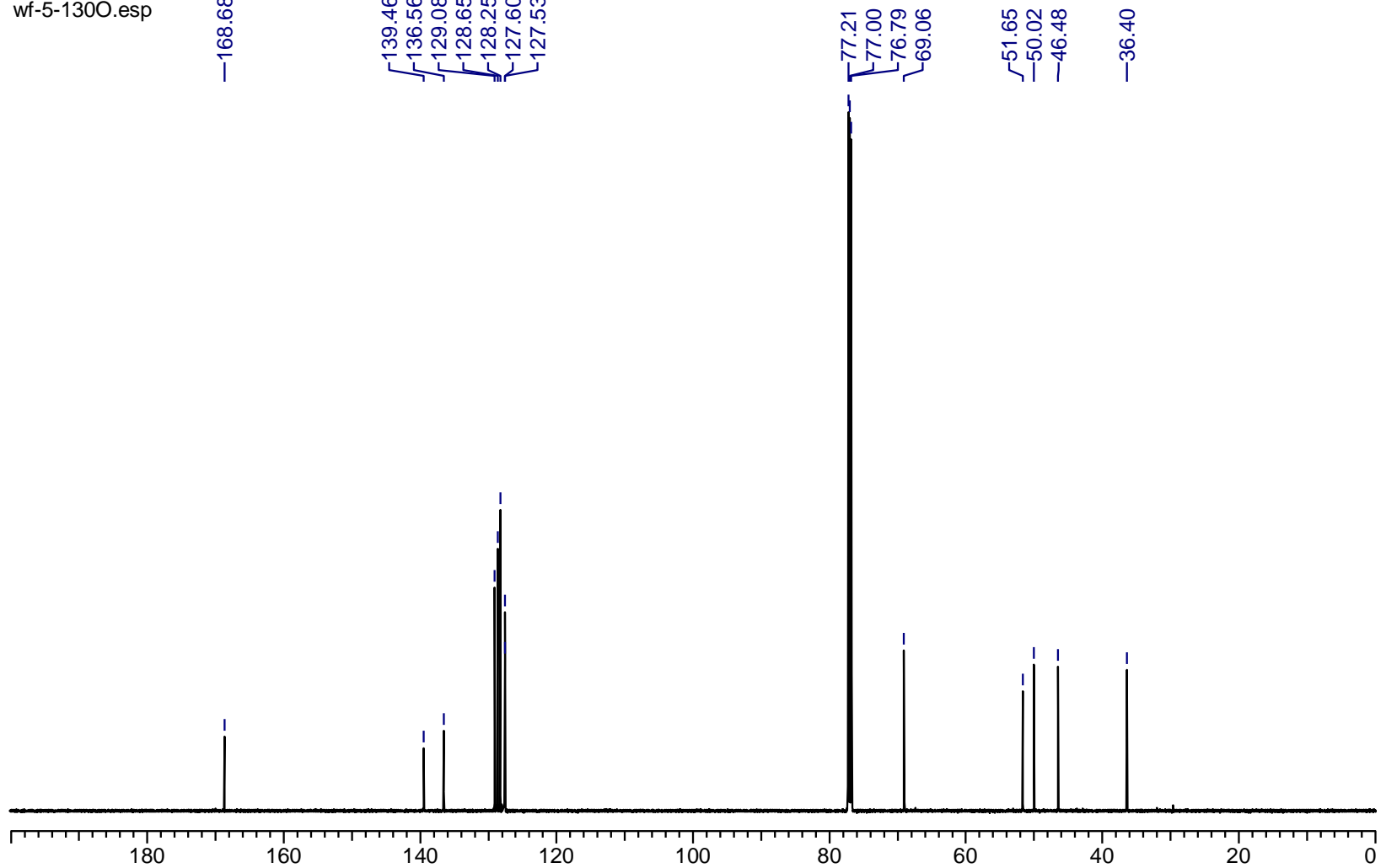

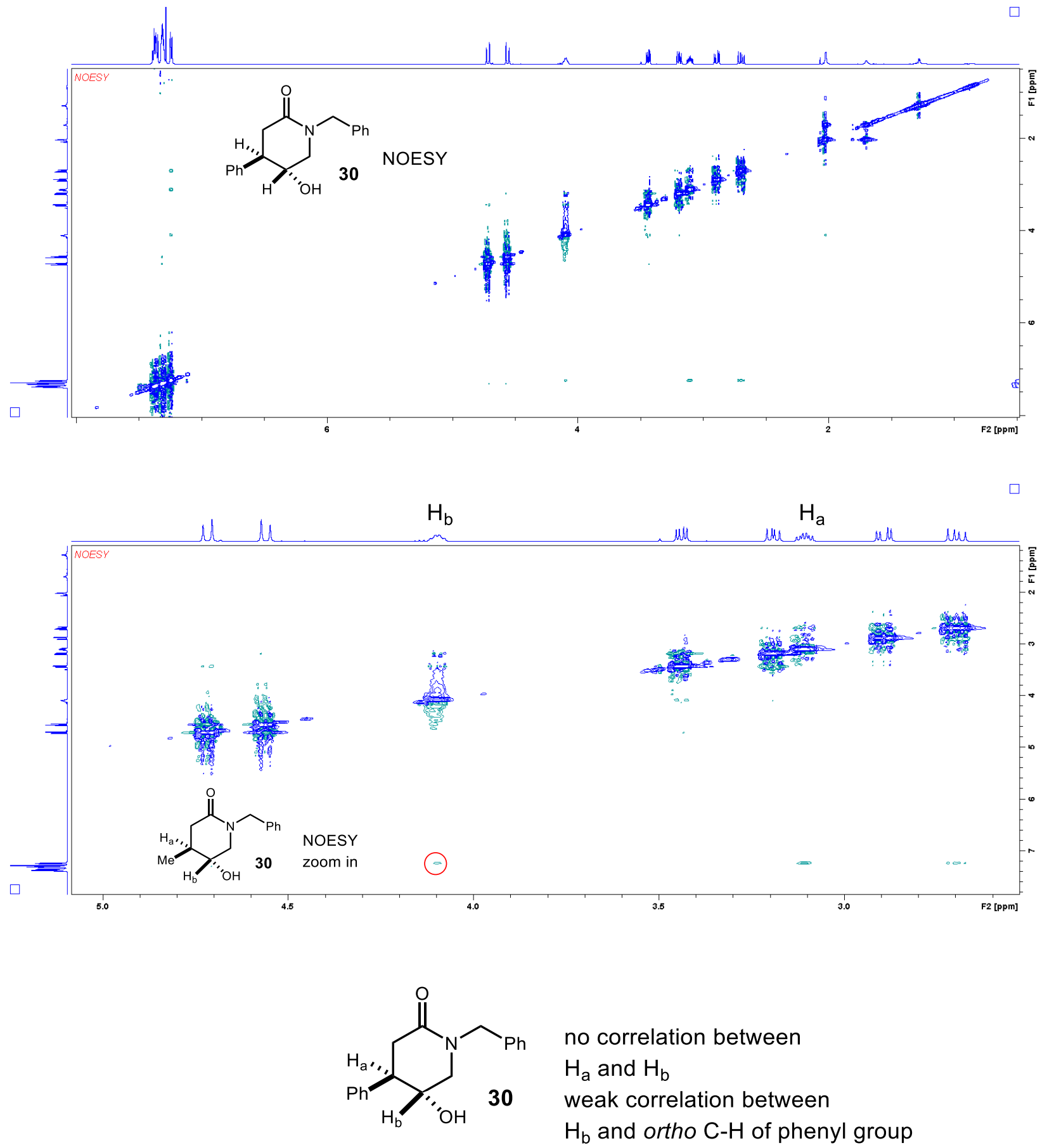
<smiles>CC1CC(=O)N(Cc2ccccc2)CC1O</smiles>

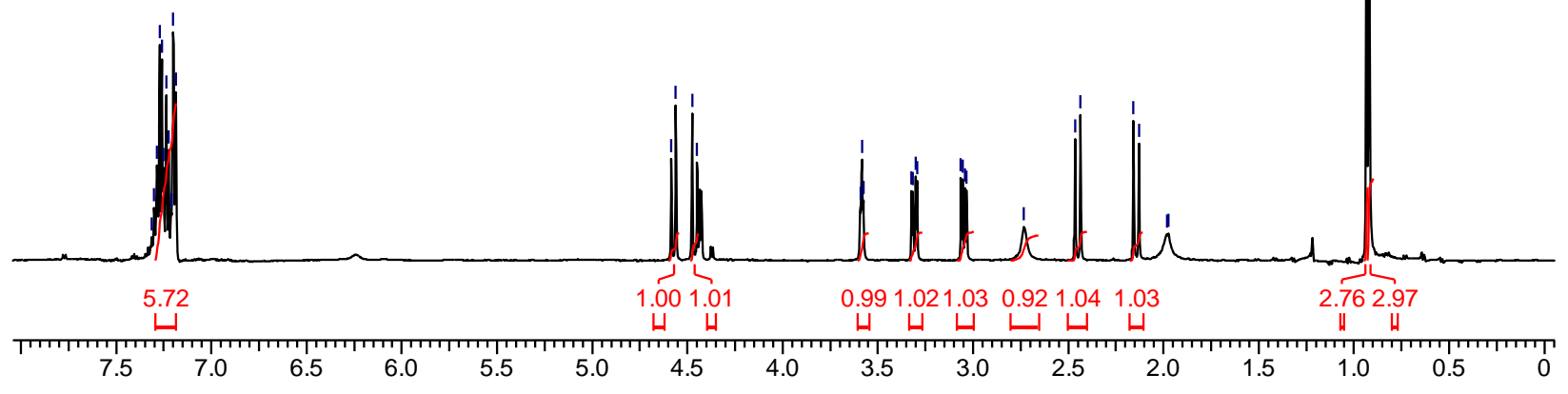

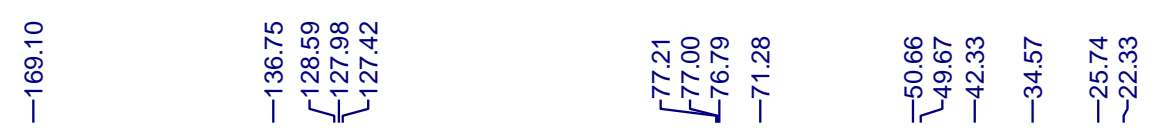

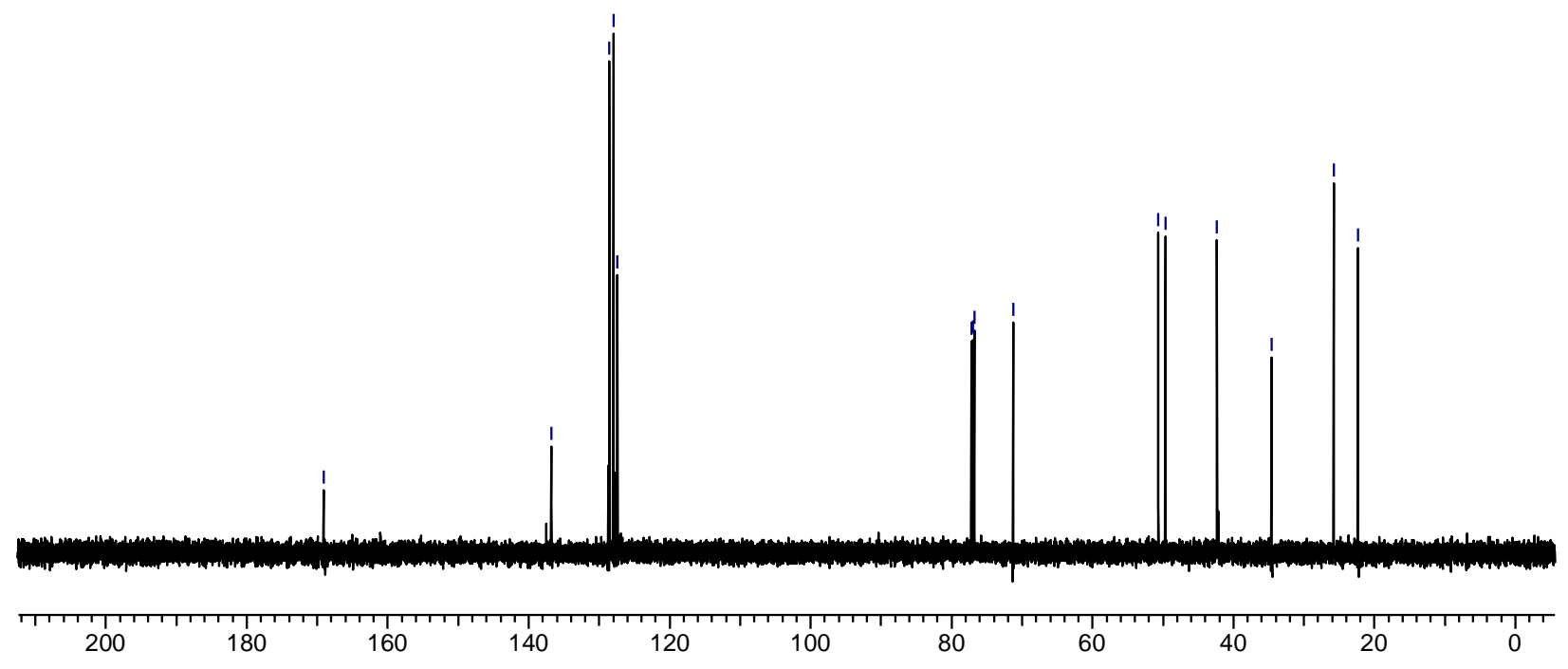


<smiles>O=C1N(Cc2ccccc2)CC(O)CC12CCCCC2</smiles>

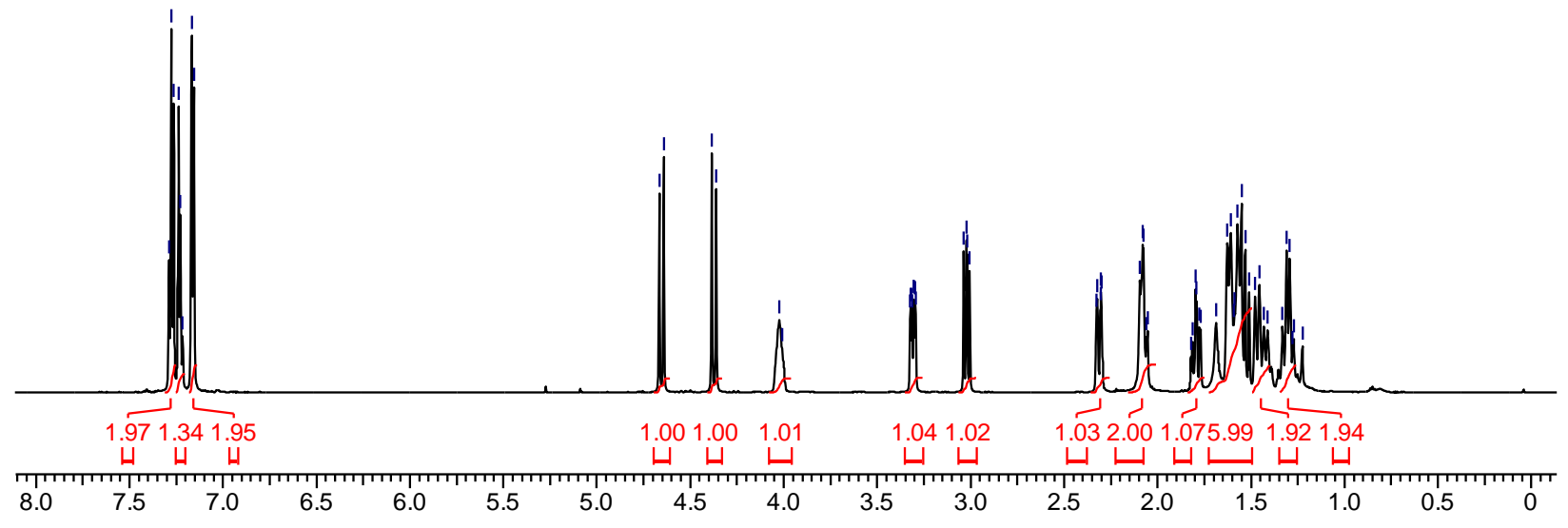

JA-31-C.001.esp是

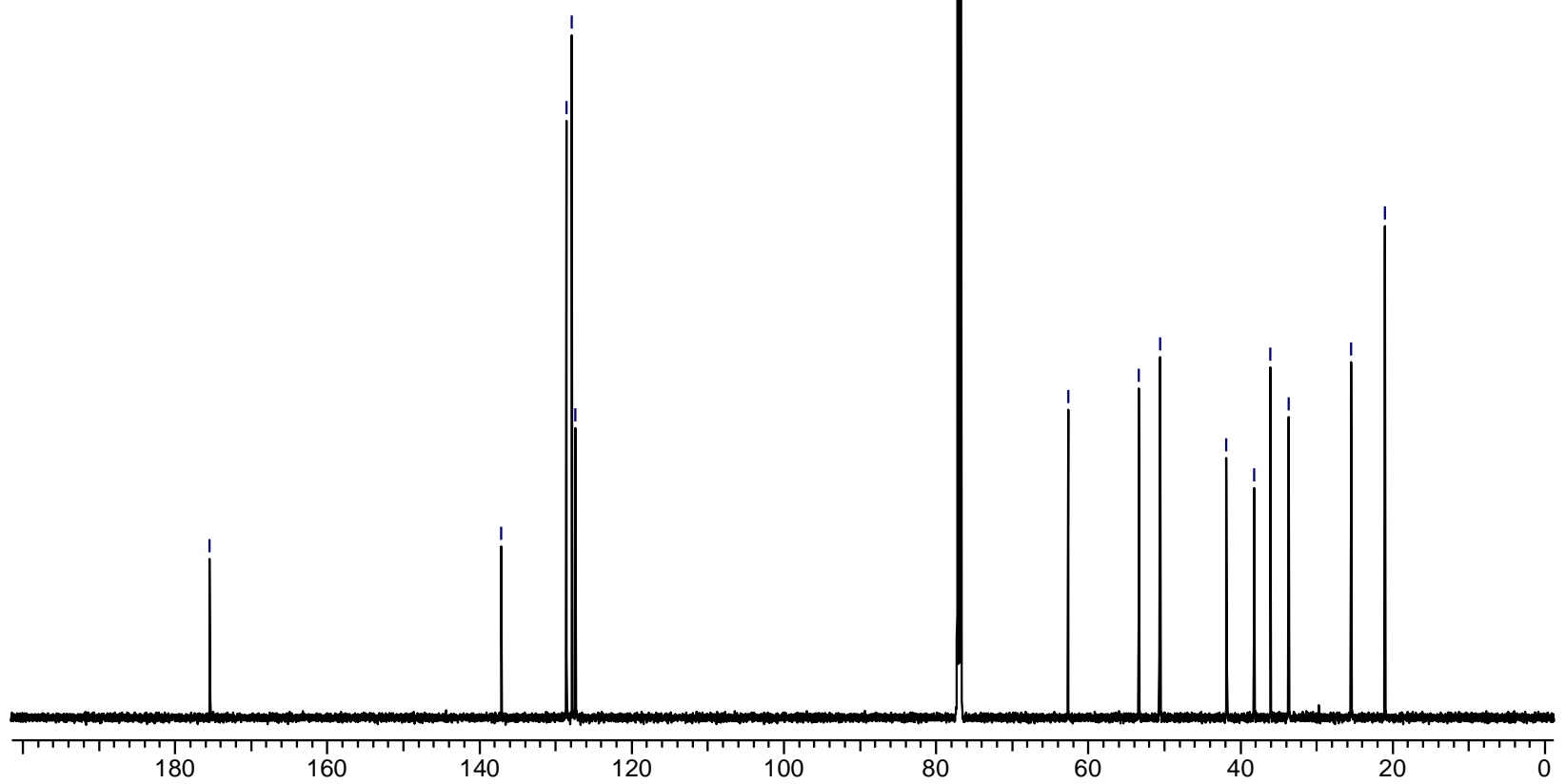



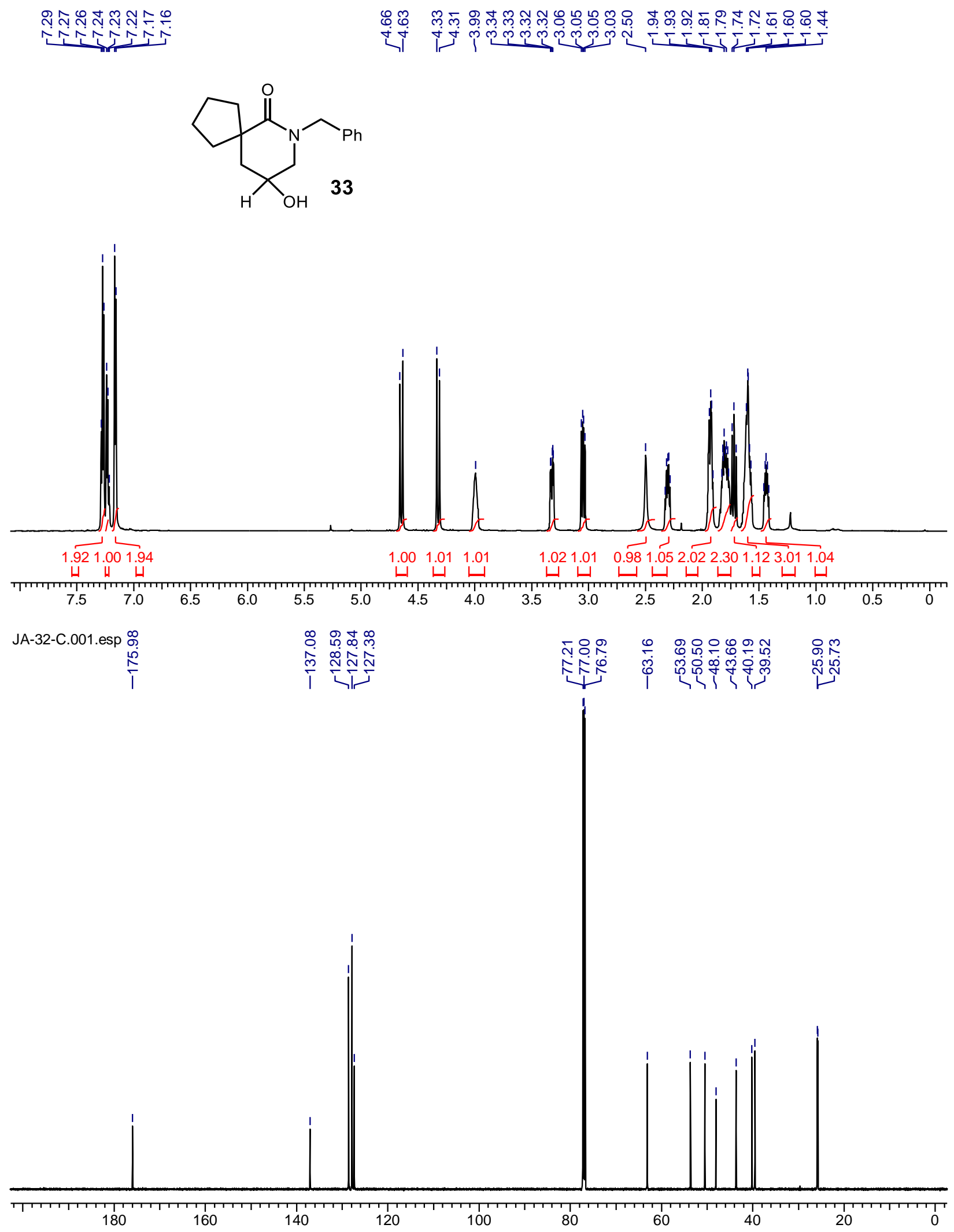
ำ

손서섯<smiles>CC(C)(C)OC(=O)N1CCC2(CC1)CC(O)CN(Cc1ccccc1)C2=O</smiles>

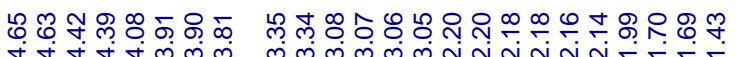

ن पंن

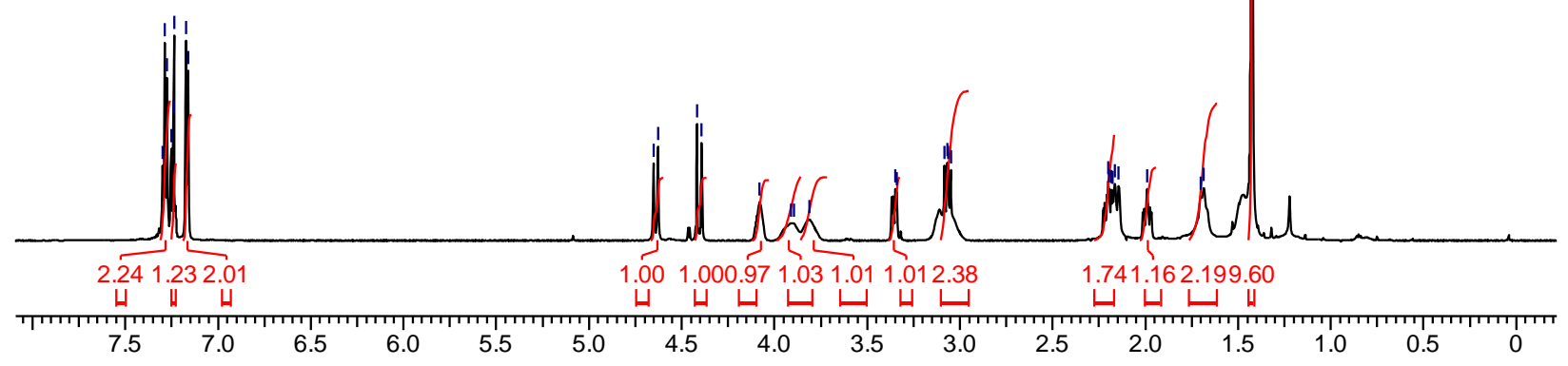

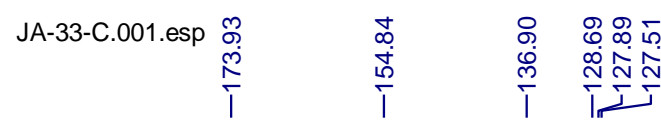

诲

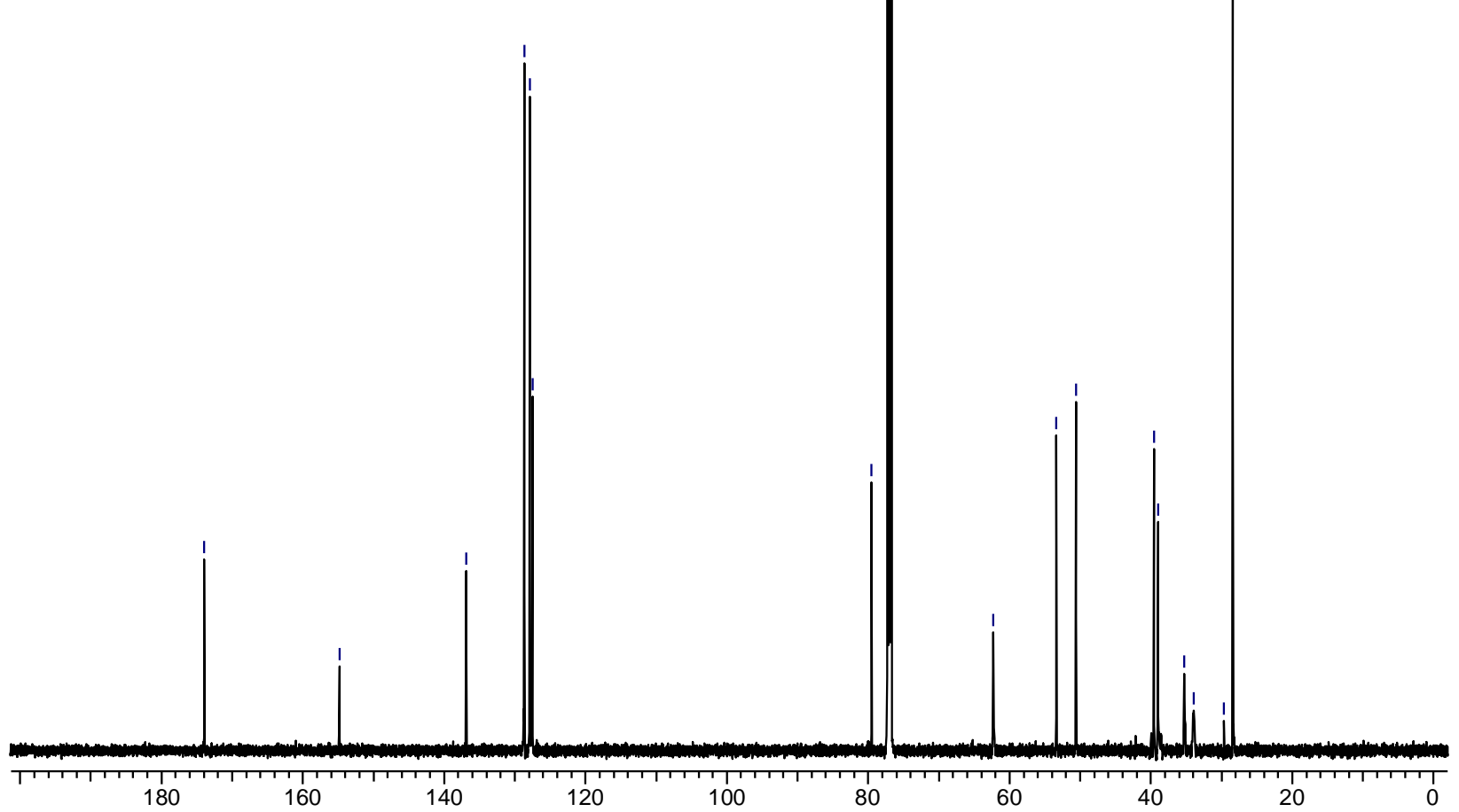




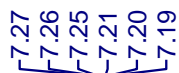<smiles>O=C1C[C@@H]2CCCC[C@]2(O)CN1Cc1ccccc1</smiles>
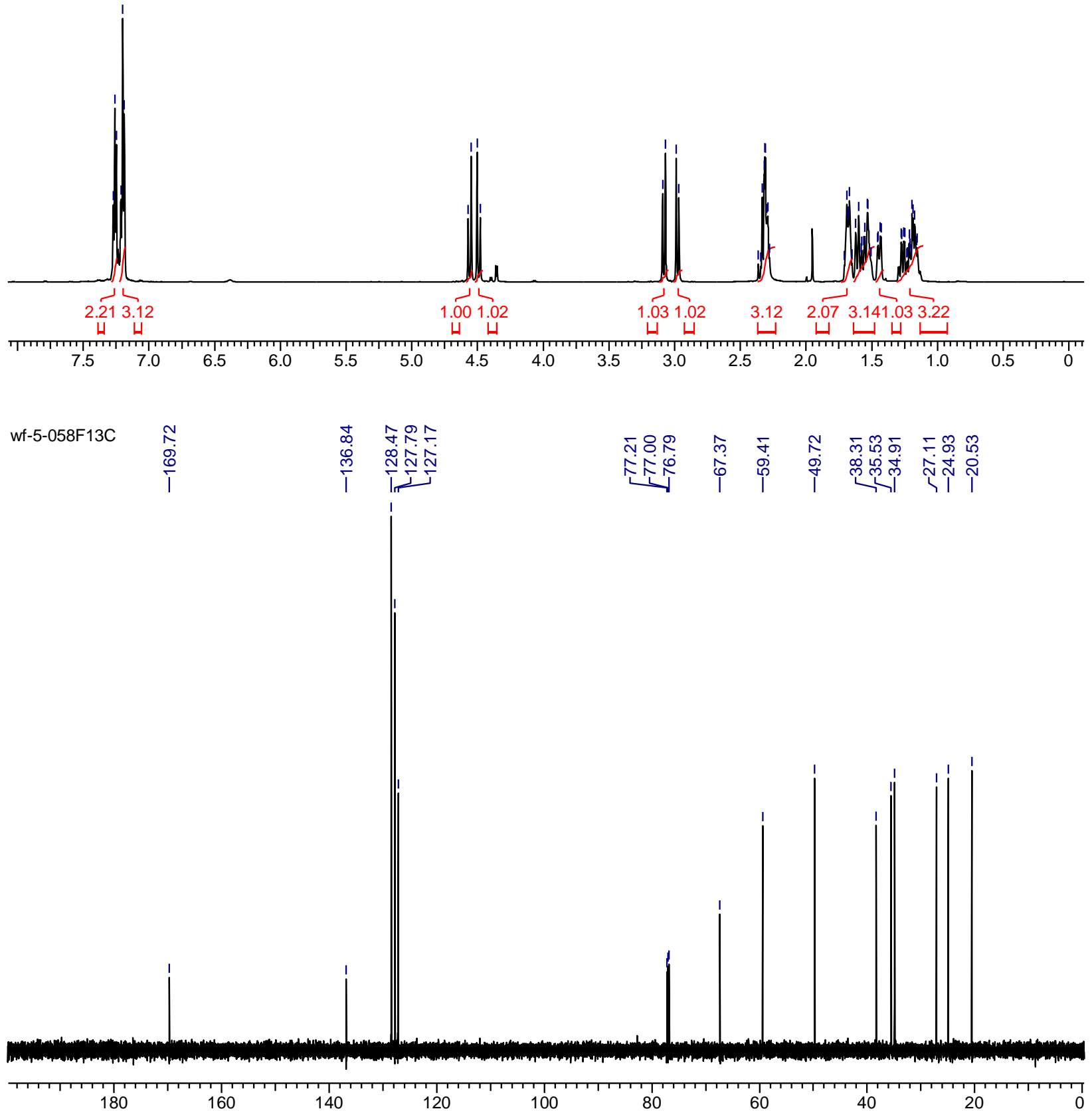

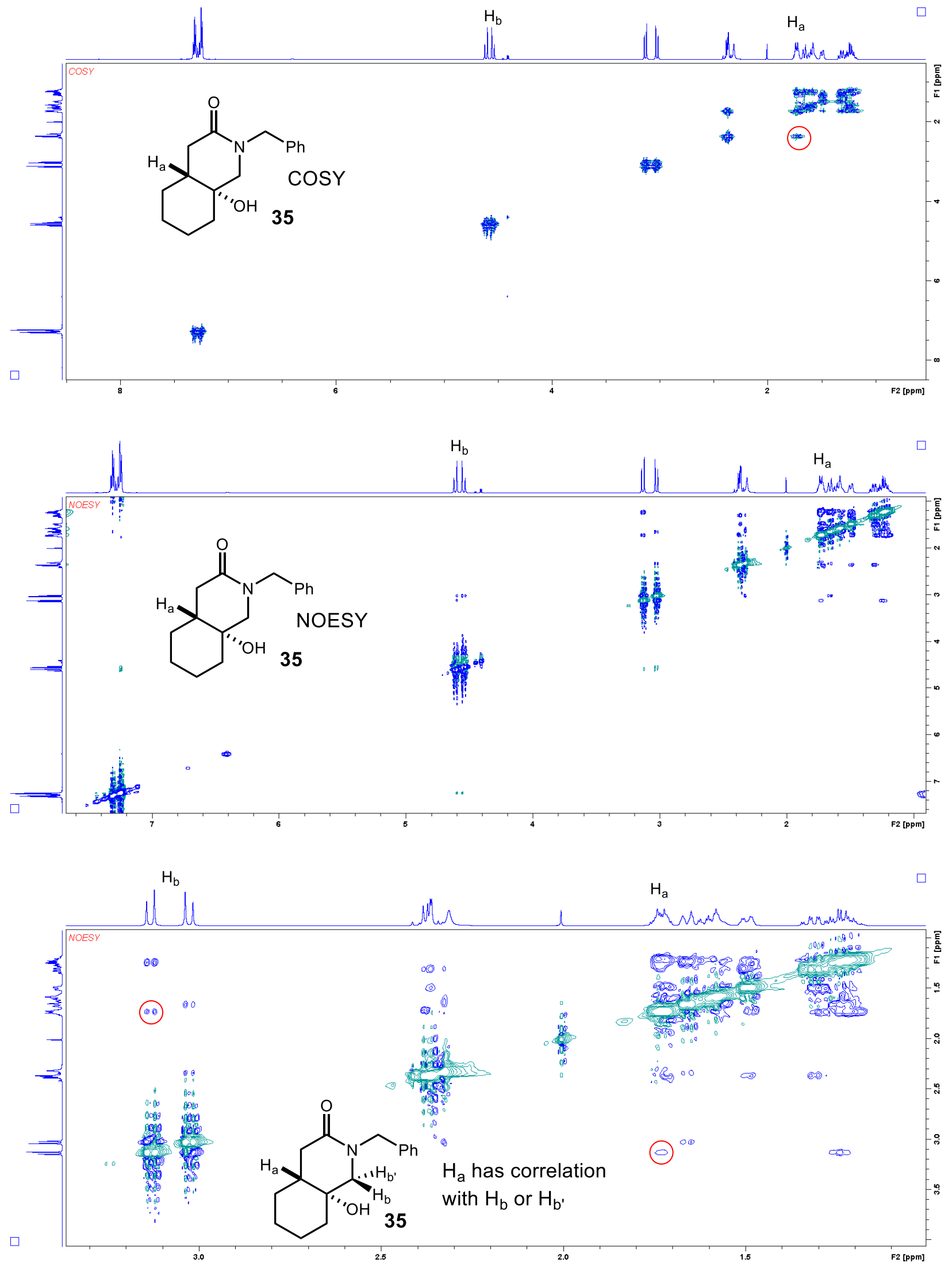

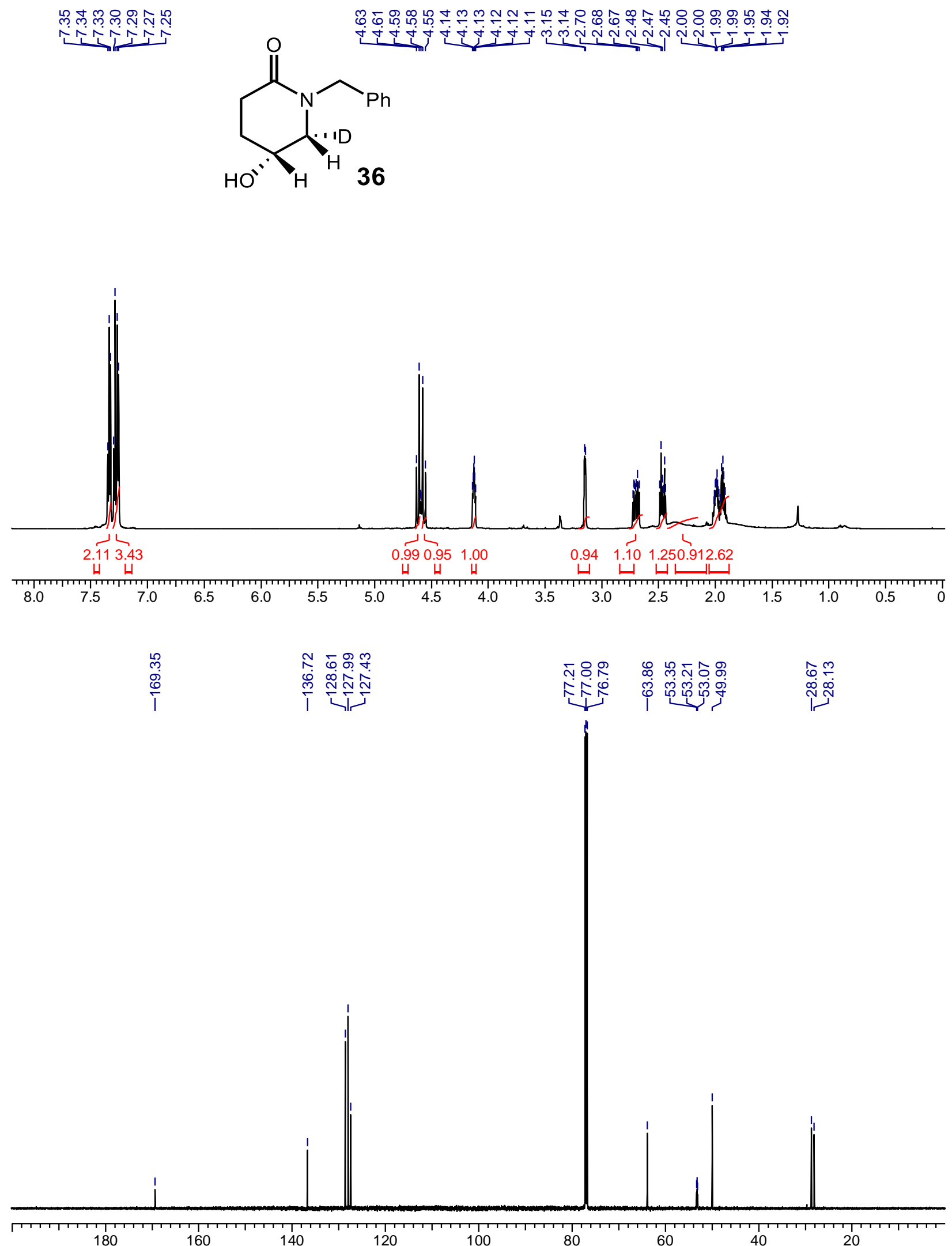

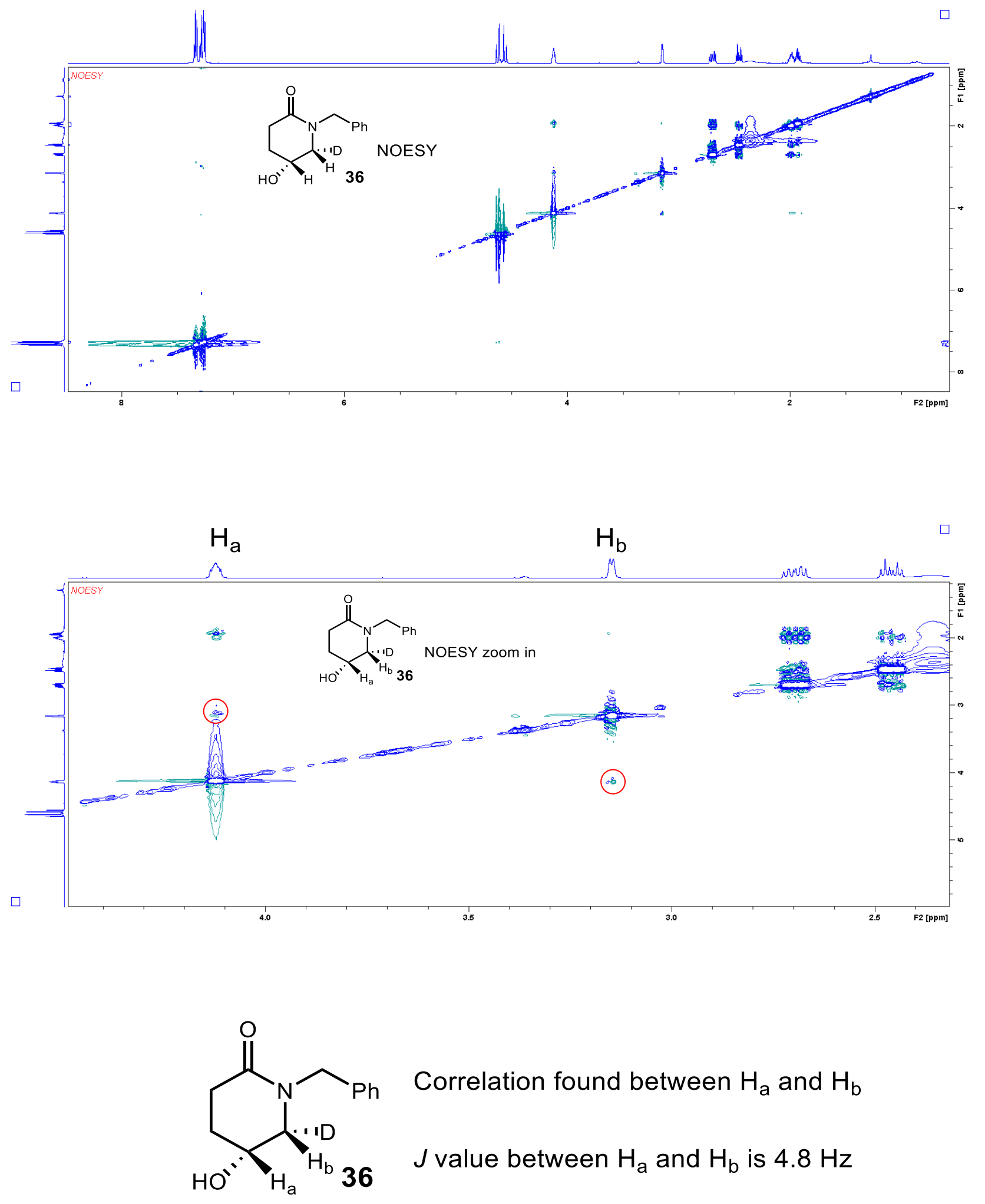


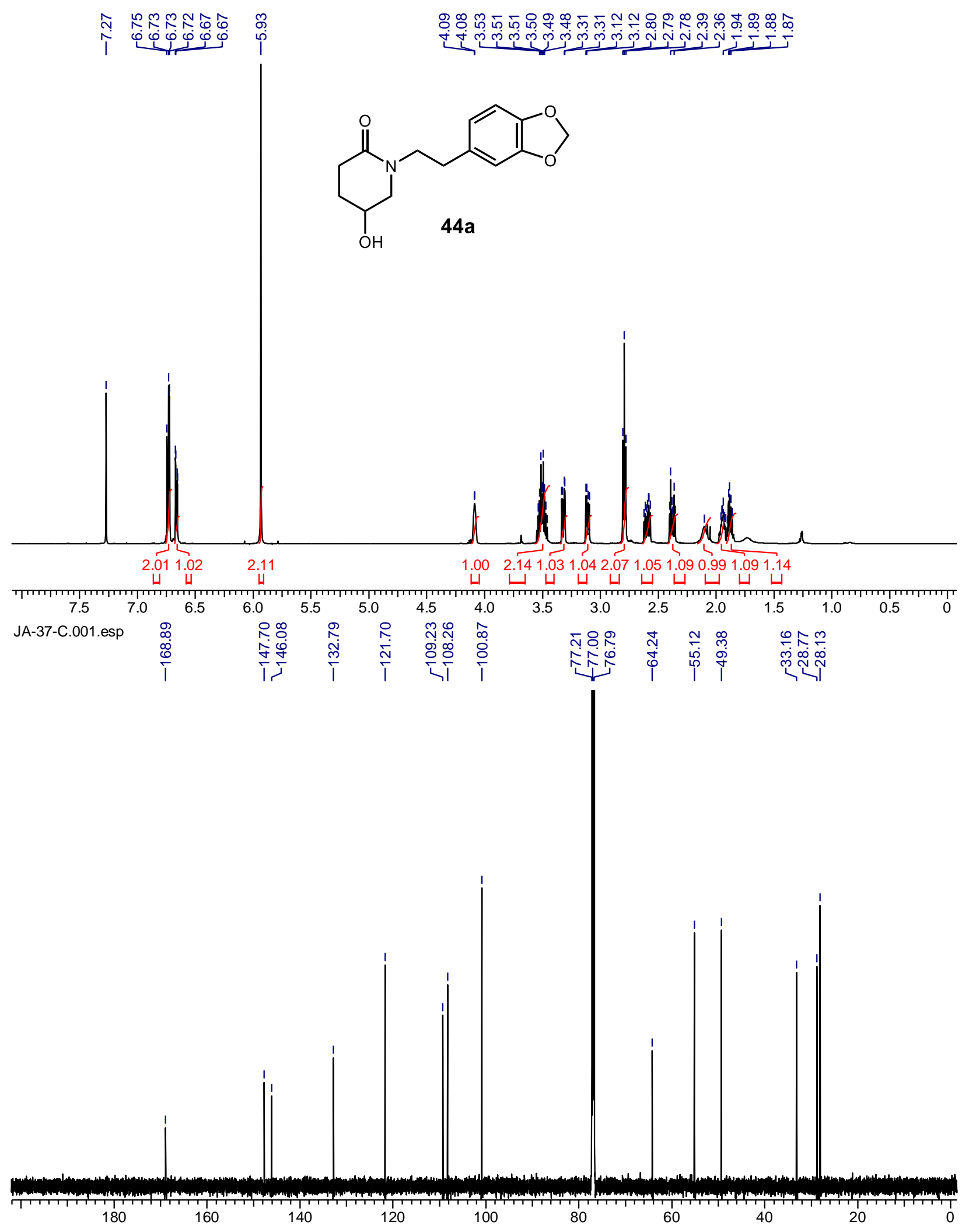




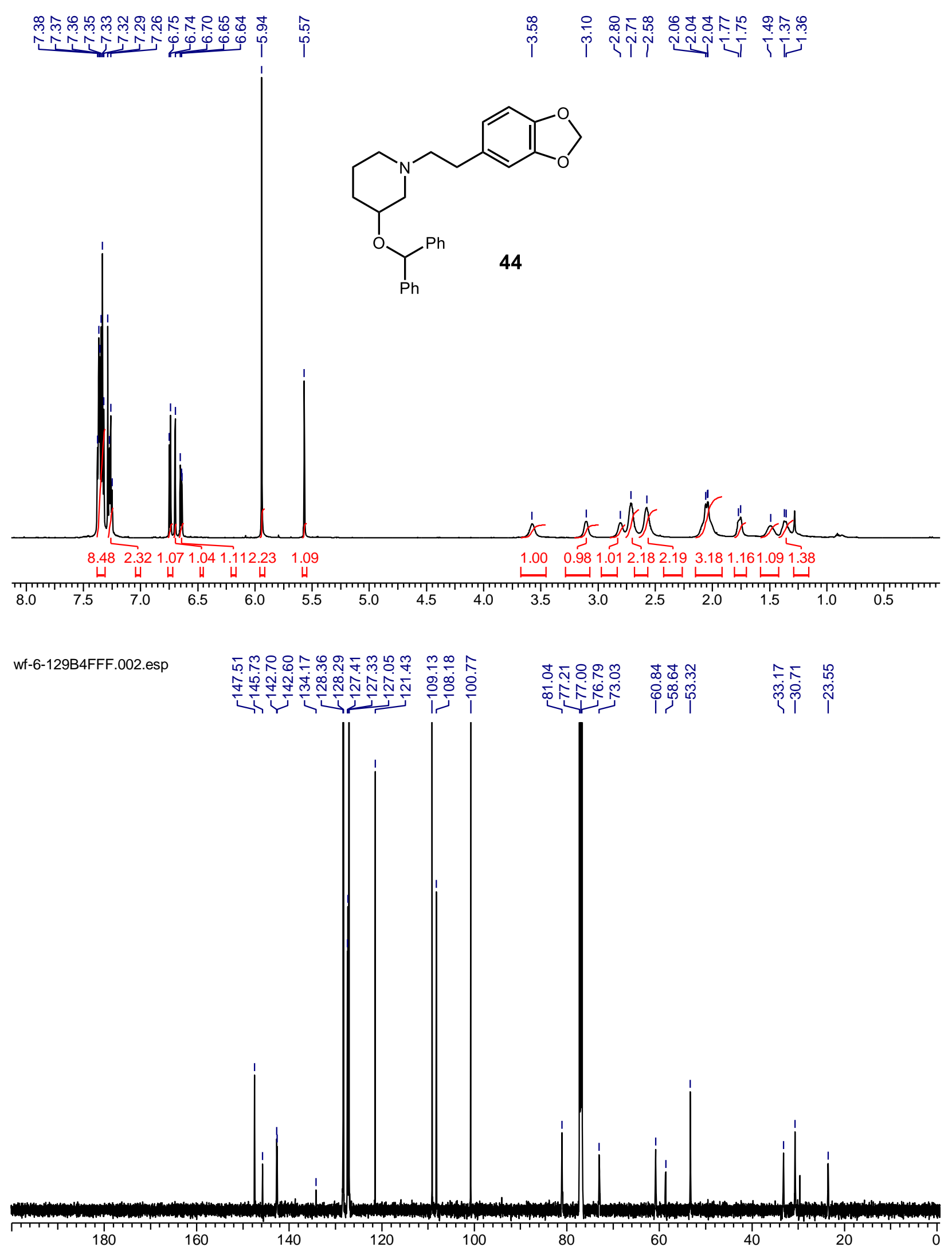

